\title{
Modelos matemáticos para evolução social: de cooperação à diversidade linguística
}

\author{
Cinthia Marie Tanaka
}

TESE APRESENTADA

$\mathrm{AO}$

Instituto de Matemática e EstatísticA

DA

Universidade DE SÃo PAUlo

PARA

OBTENÇÃO DO TÍTULO

$\mathrm{DE}$

Doutor EM CIÊNCIAS

\author{
Programa: Matemática Aplicada \\ Orientador: Prof. Dr. Renato Vicente
}

Durante o desenvolvimento deste trabalho

o autor recebeu auxílio financeiro da CAPES

São Paulo

Agosto de 2018 


\section{Modelos matemáticos para evolução social: de cooperação à diversidade linguística}

Esta versão da tese contém as correções e alterações sugeridas pela Comissão Julgadora durante a defesa da versão original do trabalho, realizada em 13/08/2018. Uma cópia da versão original está disponível no Instituto de Matemática e Estatística da Universidade de São Paulo.

Comissão Julgadora:

- Prof. Dr. Renato Vicente (orientador) - IME-USP

- Prof. Dr. Julio Michael Stern - IME-USP

- Prof. Dr. Paulo Roberto Guimarães Junior - IB-USP

- Prof. Dr. Fernando Fagundes Ferreira - EACH-USP

- Prof. Dr. Nestor Caticha - IF-USP 


\title{
Mathematical models for social evolution: from cooperation to language diversity
}

\author{
Cinthia Marie Tanaka
}

\author{
DOCTORAL THESIS SUBMITTED \\ TO \\ The Institute of MAthematics And Statistics \\ $\mathrm{OF}$ \\ UNIVERSITY OF SÃO PAULO \\ FOR \\ OBTAINING THE TITLE \\ $\mathrm{OF}$ \\ DOCTOR IN SCIENCES
}

\author{
Program: Applied Mathematics \\ Supervisor: Prof. Dr. Renato Vicente \\ During the development of this $\mathrm{PhD}$ thesis \\ the author received financial support from CAPES \\ São Paulo \\ August 2018
}





\section{Agradecimentos}

Em primeiro lugar, gostaria de agradecer a meu orientador, Renato Vicente, por me guiar e ser capaz de me encorajar por todos esses anos desde que eu era uma estudante de graduação. Além disso, sou grata ao Dr. Yoh Iwasa, que foi meu orientador quando fui estudante pesquisadora em 2011 e também durante meu estágio de pesquisa durante o doutorado, por me motivar a ser uma boa pesquisadora. Minha amiga, e co-orientadora, Joung-Hun Lee também auxiliou nessa tarefa, fazendo com que eu tivesse a melhor experiência possível durante minha estadia na Universidade de Kyushu.

Sinto-me muito feliz por ter muitos bons amigos em minha vida (Carlos, Will, Gabi, Le, Léo, Rafael, Sheng), com quem compartilho tantas conversas sobre assuntos (majoritariamente) não-acadêmicos. Alguns dos amigos que conheci durante o doutorado (Chris, Carol, Josefran, Laura, Jun, Masayuki) contribuíram muito para meu crescimento pessoal e, apesar de não os encontrar com frequência, ainda me sinto muito próxima a eles. Além disso, foi e tem sido inspirador estar em um ambiente com pessoas tão entusiasmadas com seus trabalhos, e eu agradeço aos meus colegas da Física, e em particular ao grupo de pesquisa CNAIPS-USP, e aos meus colegas do Elo7 por me fornecerem esse ambiente.

Também gostaria de agradecer o apoio incondicional de minha família durante todos os estágios do curso de doutorado. Sou particularmente grata a meus pais, que me possibilitaram a escolha dessa carreira, mesmo sem ter familiaridade com o meio acadêmico.

Agradeço também o suporte financeiro provido pela CAPES. Além disso, também gostaria de agradecer à Universidade de São Paulo por me fornecer auxílio financeiro para participar do Encontro da Sociedade de Biologia Matemática Japonesa em 2015, e à Universidade de Kyushu, conjuntamente ao laboratório de Biologia Matemática da mesma universidade, pelo auxílio financeiro recebido durante meu estágio de pesquisa no Japão. 


\section{Acknowledgements}

First, I would like to thank my supervisor Renato Vicente, for his guidance and for being able to encourage me through all these years since I was an undergraduate student. Also, I would like to thank Dr. Yoh Iwasa, who was my supervisor as a research student back in 2011 and also during my $\mathrm{PhD}$ research internship, in 2016, for motivating me to be a good researcher. My friend, and co-supervisor, Joung-Hun Lee also assisted in this task, and provided me the best I could expect during my stay at Kyushu University.

I feel very lucky for having many best friends in my life (Carlos, Will, Gabi, Le, Léo, Rafael, Sheng), with whom I have shared so many interesting conversations about (mainly) non-academic things. Some of the friends I met through the PhD course (Chris, Carol, Josefran, Laura, Jun, Masayuki) contributed a lot to my personal growth and, although I do not have the chance to meet them frequently, I still feel very close to them. It has been inspiring to be at an environment where people are so enthusiastic about their work, and I thank my colleagues at the Physics Institute, and in particular the research group CNAIPS-USP, and my colleagues at Elo7 for providing me such environment.

I am also very grateful for having the support of my family and friends during all stages of my $\mathrm{PhD}$ course. I am particularly thankful to my parents, who enabled me to take this career path, even though they were not familiar with the academic world..

Financial support from CAPES is acknowledged. I also thank University of São Paulo for providing me financial support to attend the Mathematical Biology Japanese Society Meeting in 2015 and Kyushu University along with the Mathematical Biology Laboratory from Kyushu University for the financial support during my PhD research internship in Japan. 


\section{Resumo}

Uma das características que nos distinguem de outros seres vivos é nossa cultura. Entretanto, como comportamentos não fossilizam, é difícil reconstruir o passado para gerar insights sobre por que nos tornamos o que somos hoje. Juntamente com dados etnográficos e experimentais, os modelos matemáticos têm sido utilizados para abordar a questão sobre como nossos comportamentos foram moldados pela evolução.

Esta tese está dividida em duas partes. Na primeira parte, discutiremos sobre seleção multinível e sobre como o framework matemático chamado Two-level Fisher Wright (TLFW) pode nos ajudar a entender a evolução da cooperação em populações humanas. Após descrevermos o problema da cooperação através do uso de ferramentas de teoria dos jogos, revisamos algumas das teorias atuais sobre por que a cooperação evoluiu. Em seguida, empregamos o framework TLFW ao problema da emergência de altruísmo em populações de caçadores-coletores, considerando uma situação em que o conflito entre grupos direciona a seleção.

Na segunda parte, abordamos o tópico de diversidade linguística e apresentamos a importância de se estudar a competição entre línguas para ajudar a preservá-las. Traçando um paralelo entre a evolução das línguas e a evolução de normas sociais, introduzimos um modelo para analisar a persistência de dialetos, quando existe competição com uma língua padrão nacional. 


\section{Abstract}

One of the features that distinguish human beings from other living species is our culture. However, since behaviors do not fossilize, it is difficult to reconstruct the past to get insights about why we are who we are. Along with ethnographic and experimental data, mathematical models have been used to address the question of how our behaviors were shaped by evolution.

This thesis is divided into two parts. In the first part, we will discuss multilevel selection and how the mathematical framework Two-Level Fisher-Wright (TLFW) can help us to understand the evolution of cooperation in human populations. After describing the problem of cooperation by using game theory, we review some of the present theories about why cooperation has evolved. Then, we apply the TLFW framework to the problem of the evolution of altruism in populations of hunter-gatherers, considering a situation in which group conflict drives selection.

In the second part, we discuss language diversity and present the importance of studying the competition between languages for helping to preserve them. By drawing a parallel between the evolution of language and social norms, we introduce a mathematical model to analyze the persistence of dialects competing against a national standard language. 
If you never noticed, it never happened.

Haruki Murakami (1Q84) 


\section{Contents}

Acknowledgements $\quad$ i

$\begin{array}{lll}\text { Abstract } & \text { ii }\end{array}$

List of Tables $\quad$ xi

$\begin{array}{lc}\text { List of Figures } & \text { xix }\end{array}$

1 Introduction $\quad 1$

1.1 Motivation and Main Results . . . . . . . . . . . . . . . . . . . 2

1.1.1 Evolution of altruism . . . . . . . . . . . . . . . . . . . 2

1.1 .2 Language evolution . . . . . . . . . . . . . . . . . . . . 3

1.2 Thesis Structure . . . . . . . . . . . . . . . . . . . . 4

2 Mechanisms for the evolution of cooperation $\quad 5$

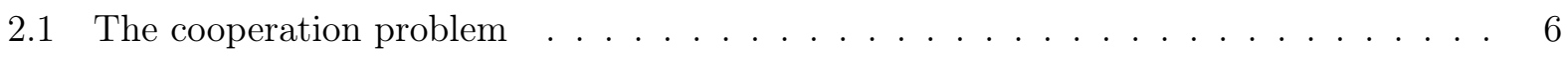

2.1 .1 Prisoner's Dilemma . . . . . . . . . . . . . . . . . . . . . . 7

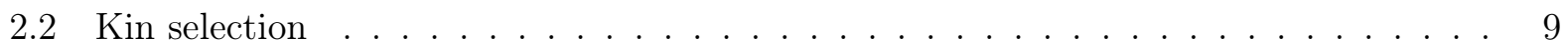

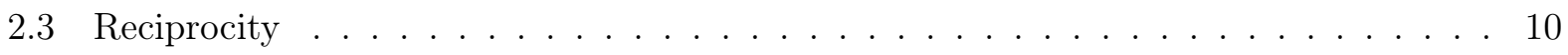

2.3 .1 Repeated games . . . . . . . . . . . . . . . . . . . 11

2.3.2 Evolving direct reciprocity . . . . . . . . . . . . . . . . . . . . . . . 12 
2.3.3 Evolving indirect reciprocity . . . . . . . . . . . . . . . . . . . . . 14

2.4 Multilevel selection . . . . . . . . . . . . . . . . . . . . . . . . 18

2.4 .1 The Price equation . . . . . . . . . . . . . . . . . . . . 21

2.4.2 From Price equation to Hamilton's rule . . . . . . . . . . . . . . . 23

$3 \quad$ Two-level Fisher-Wright model: a general framework $\quad 27$

3.1 The model . . . . . . . . . . . . . . . . . . . . . . . . . 27

3.1 .1 Evolutionary stages . . . . . . . . . . . . . . . . . 32

3.2 Early Stage approximations . . . . . . . . . . . . . . . . . . . 33

3.2 .1 Limit of weak selection . . . . . . . . . . . . . . 36

3.2.2 Limit of large $n$ and small $m$ under weak selection . . . . . . . . . . 40

3.3 Examples of applications . . . . . . . . . . . . . . . . 43

3.4 Discussion - when relatedness is not enough . . . . . . . . . . . . . . . 47

3.4.1 A practical example - contingent cooperation . . . . . . . . . . . . 48

3.4.2 A general way of calculating the condition for the invasion of rare mutants . . 52

4 Evolving altruism in the presence of intergroup conflicts $\quad 54$

4.1 Related work and motivation . . . . . . . . . . . . . . . . 54

4.2 The model . . . . . . . . . . . . . . . . . . . . . . . . . 55

4.2.1 Deriving an analytical condition for the emergence of altruism . . . . . . . . 59



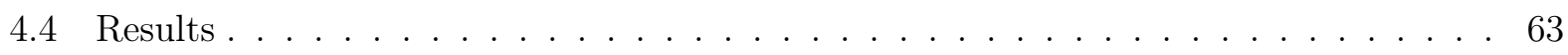

4.4.1 Comparing results from analytical derivation . . . . . . . . . . 63

4.4 .2 Using realistic parameter values . . . . . . . . . . . . . . 64

4.4.3 Comparing simulation results with the eigenvalue method . . . . . . . 65

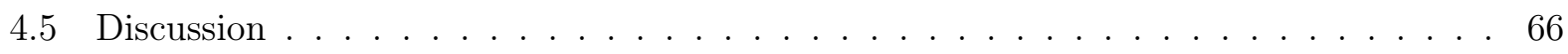


5.1 Related work and motivation . . . . . . . . . . . . . . . . . 69

5.2 Methods . . . . . . . . . . . . . . . . . . . . . 71

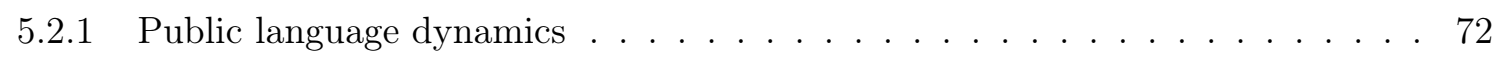

5.2.2 Private language dynamics with peer pressure model of conformity . . . . . . 73

5.2.3 Private language dynamics with an alternative model of conformity - the unanimity pressure model . . . . . . . . . . . . . . . . . . . 79

5.3 Combining the public and private language dynamics $\ldots \ldots \ldots \ldots$

5.3.1 Combined language dynamics with peer pressure model of conformity . . . . 80

5.3.2 Combined language dynamics with unanimity pressure model of conformity . 81

5.4 Results . . . . . . . . . . . . . . . . . . . . . . 82

$5.4 .1 \quad$ Peer pressure model $\ldots \ldots \ldots \ldots$. . . . . . . . . . . . . . 83

5.4 .2 Unanimity pressure model . . . . . . . . . . . . . . . . . 85

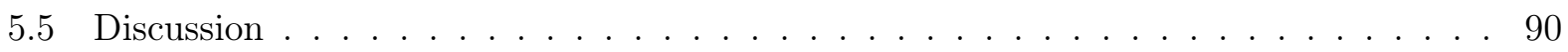

6 Conclusions and perspectives $\quad 94$

$\begin{array}{lr}\text { Appendices } & 96\end{array}$

$\begin{array}{ll}\text { A Short introduction to evolutionary game theory } & 97\end{array}$

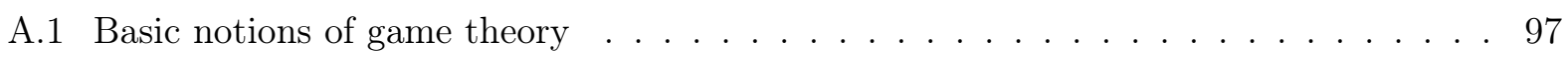

A.1.1 Public good games . . . . . . . . . . . . . . . . . . . 99

A.2 Basic notions of evolutionary game theory . . . . . . . . . . . . . . . . 102 


\section{List of Tables}

2.1 Categories of social behavior according to the interaction between actor and recipient. 6

2.2 The leading eight strategies. For each strategy (columns numbered from 1 to 8 ), both assessment rules (first 8 rows) and action rules (last 4 rows) are shown. Assessment rules situations are represented showing the reputation of the donor (Good, G, or $B a d, B)$, an arrow which may indicate whether the donor helped $(\rightarrow)$ or not $(\nrightarrow)$ the recipient, and the reputation of the recipient $(G$ or $B)$. Action rules are represented showing the reputation of the donor $(G$ or $B)$, an arrow $\stackrel{?}{\rightarrow}$ and the reputation of the recipient; possible actions are $Y$ (to help) or $N$ (to refuse to help). . . . . . . . 18

2.3 Symbols used in Price equation derivation. . . . . . . . . . . . . . . . . 21

A.1 Summary table with the payoffs for SRPG and OOPG. . . . . . . . . . . . 100 


\section{List of Figures}

2.1 Examples of assessment rules. In SCORING, reputations do not matter, and a donor $(D)$ who helps a recipient $(R)$ is always labeled $\operatorname{Good}(G)$, while donors who do not help recipients are always labeled $B a d(B)$. In STANDING, the difference from the SCORING rule is that a $G$ donor who refuses to help a $B$ recipient is still considered to be $G$. In JUDGING, the difference from the STANDING rule is that helping a $B$ recipient makes a donor to be labeled as $B \ldots \ldots \ldots \ldots$

2.2 Examples of action rules. In $\mathrm{CO}$, only the reputation of the recipient $(R)$ is used to make the decision of helping $(Y)$ or not $(N)$ an individual: if the recipient is Good $(G)$, the donor $(D)$ helps him, otherwise, if the recipient is $B a d(B)$, the donor will refuse help to him. In SELF, the decision is based solely on the reputation of the donor: a donor helps a recipient only if his own reputation is $B$. In AND, a donor helps a recipient only if his own reputation is $B$ and if the reputation of the recipient is $G$. In OR, a donor helps a recipient if his own reputation is $B$ or if the reputation of the recipient is $G \ldots \ldots \ldots \ldots \ldots \ldots \ldots \ldots$

3.1 Population structure in TLFW. Individuals are divided in a large number $g$ of groups. Each group contains $n$ individuals, that are classified as $A$ or $N$, according to their actions in the interactions inside their groups. . . . . . . . . . . . . . . 28

3.2 Individual reproduction in TLFW. The fraction of individuals of a label $A$ or $N$ in the next generation is proportional to the fraction of individuals $A$ or $N$ in the current generation weighted by $w_{k}^{A} / \bar{w}_{k}$ or $w_{k}^{N} / \bar{w}_{k} \ldots \ldots \ldots \ldots \ldots$

3.3 Group reproduction in TLFW. The average number of groups which has a parent group of type $k$ is given by the average fitness of individuals inside this group, $\bar{w}_{k}$. $\quad 29$

3.4 Detailed explanation about the formalization of the selection process in the terms of matrix $S$ from eq. $(3.3) \ldots \ldots \ldots \ldots \ldots \ldots$ 
3.5 Detailed explanation about the formalization of the migration process in the terms of matrix $M$ from eq. (3.4) . . . . . . . . . . . . . . . . . . . . . . . 30

3.6 An evolutionary timeline representing the stages to which social trait $A$ is subject, when it starts from a single mutation at VES inside a monomorphic population dominated by trait $N$. In ES, the fate of the mutation $A$ is decided and $A$ can be extinct or survive with its distribution reaching a demographic equilibrium at SES. LS occurs when the number of individuals of trait $A$ cannot be neglected compared to the total number of groups $g \ldots \ldots \ldots \ldots \ldots \ldots$

3.7 Simplified migration process when individuals $A$ are very rare in the population. We assume that, in the migration process, it is very unlikely that a group of type $k>0$ receives a migrant individual of trait $A$.

3.8 Explanation about the calculation of $K_{u}$, the number of individuals who are IBD in a group to which the chosen focal individual of trait $A$ belongs, at current generation $u$, from the number of individuals of trait $A$ in the previous generation $u-1, K_{u-1}$. The focal individual is represented by the orange dot, while her ancestral in the previous generation is the green dot. In the left, we depict the situation when the focal individual migrates to another group: since $p \ll 1$, she arrives to a group in which she is the only individual with trait $A$, so that $K_{u}=1$. In the right, the assumption of $\delta=0$ determines the probability $K_{u-1} / n$ with which an individual is of trait $A$ when he descends from a group with $K_{u-1}$ individuals of trait $A$. In this case, the current generation will have $K_{u}$ individuals who are IBD if their parents are among the $K_{u-1}$ individuals of the previous generation and are not subject to migration

3.9 Fitness functions for an altruist individual $(A)$ and a non-altruist individual $(N)$ versus number of altruists $(k)$ for different public goods games. At each panel, a different public goods game is considered: from left to right, respectively, OOPG (Others-Only Public Goods Game), IPG (Iterated Public Goods Game) and THR (Threshold Public Goods Game). For all public goods games, we considered $n=20$ and $\delta=0.1$. OOPG parameters: $b=5, c=1$; IPG parameters: $b=5, c=1$, $T=10, \theta=8$; THR parameters: $b=10, b^{\prime}=10, c=1, \theta=4 \ldots \ldots \ldots$ 
3.10 Average fitness $\left(\bar{w}_{k}\right)$ versus number of altruists $(k)$ at three different selection strength $(\delta)$ levels for different public goods games. At each panel, a different public goods game is considered: from left to right, respectively, OOPG (Others-Only Public Goods Game), IPG (Iterated Public Goods Game) and THR (Threshold Public Goods Game). For all public goods games, we considered $n=20$. OOPG parameters: $b=5, c=1$; IPG parameters: $b=5, c=1, T=10, \theta=8$; THR parameters: $b=10$,

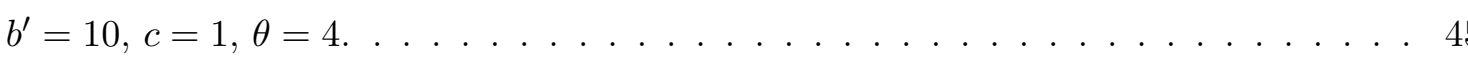

3.11 Distribution of group types $\left(\nu_{k}\right)$ for different public goods games and different values of selection strength $(\delta)$. OOPG represents the others-only public goods game; IPG is the iterative public goods game; and THR is the threshold public goods game. For all public goods games, we considered $n=20$ and $m=0.1$. OOPG parameters: $b=5, c=1$; IPG parameters: $b=5, c=1, T=10, \theta=8$; THR parameters: $b=10$,

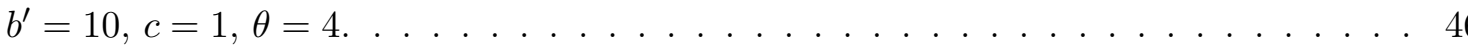

$3.12 \rho$ against different values of migration rates $(m)$ at three different selection strength $(\delta)$ levels for different public goods games. Dashed black line indicates the critical value above which altruism is viable. At each panel, a different public goods game is considered: from left to right, respectively, OOPG (Others-Only Public Goods Game), IPG (Iterated Public Goods Game) and THR (Threshold Public Goods Game). For all public goods games, we considered $n=20$. OOPG parameters: $b=5, c=1$; IPG parameters: $b=5, c=1, T=10, \theta=8$; THR parameters: $b=10$,

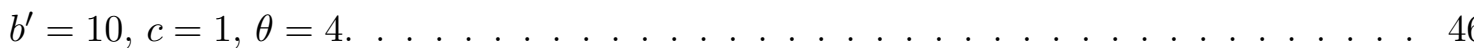

3.13 In the left panel, we see how different values of $\alpha$ and $\beta$ might lead to the same relatedness values $(r)$. In the right panel, the distribution of $k$ is plotted for two different sets of $\alpha$ and $\beta$ (represented by the star and the diamond markers), which lead to the same relatedness level, $r=0.25$. The shaded areas represent $\operatorname{Pr}(k \geq 9 \mid A)$. Parameters: $n=16, f=10$ and $m=6$.

4.1 Model scheme. First, there is war between groups. Secondly, intragroup selection takes place and individuals reproduce. Finally, individuals are allowed to migrate to

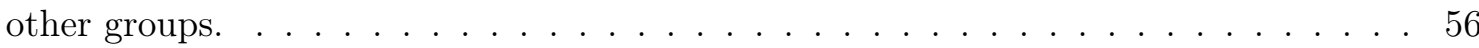


4.2 Intergroup selection in our model. Groups engage in war with probability $\beta$ and can win or lose the war according to a function $\lambda_{k}$ that depends on the number of altruists $k$ in the group. Three outcomes are possible from this process: either the group does not fight the war and keeps its deme; or it fights the war and may go to extinction or reproduce twice, at its own deme and at the opponent deme. . . . . . . 56

4.3 Critical probability of war when $c=0.03, n=26$ for different approaches of getting the viability condition.

4.4 Critical cost versus probability of war for different levels of influence of altruists in the war outcome when migration is equivalent to $r=7 \%$ and $r=15 \%$ and number of individuals in the group $n=26$. Blue area represents the set of parameters which can be considered realistic.

4.5 Comparison between simulation results and the eigenvalue method for critical migration rate $\left(m_{c}\right)$ versus probability of war $(\beta)$, when $n=26$ and for two different values of $\alpha: \alpha=0.1$ (top figures) and $\alpha=0.3$ (bottom figures). Simulation parameters: $N=5000$ (number of groups), $\mu=0.0001$ (mutation rate) and $\max _{\mathrm{IT}}=10000$ (maximum number of iterations).

5.1 Schematic figure of public language dynamics. Individuals choose their languages according to their preferences and are also influenced by the number of individuals who speak each language. $\pi_{A}$ is preference regarding speaking $A, \pi_{B}$ for preference regarding speaking $\mathrm{B}, \xi$ is the conformity factor . . . . . . . . . . . . . .

5.2 Schematic figure of peer pressure model for $n=2$. Individuals are divided in groups of 2 individuals, who can be labeled $a$ or $b$, according to the language they use in private. In the peer pressure model, the pressure to conform to one language is higher as the number of speakers of that language increases inside the group. $\pi_{a}$ is preference regarding speaking $\mathrm{A}$ in private; $\pi_{b}$ is the preference regarding speaking B in private; $\xi$ is the conformity factor. . . . . . . . . . . . . . . . . . . .

5.3 Schematic figure of peer pressure model for $n=3$. Individuals are divided in groups of 3 individuals, who can be labeled $a$ or $b$, according to the language they use in private. In the peer pressure model, the pressure to conform to one language is higher as the number of speakers of that language increases inside the group. $\pi_{a}$ is preference regarding speaking $\mathrm{A}$ in private; $\pi_{b}$ is the preference regarding speaking $\mathrm{B}$ in private; $\xi$ is the conformity factor. . . . . . . . . . . . . . . 75 
5.4 Schematic figure of unanimity pressure model. Individuals are divided in groups of 3 individuals, who can be labeled $a$ or $b$, according to the language they use in private. In the unanimity pressure model, there is pressure to conform to one language only if all the other individuals speak that language inside the group. $\pi_{a}$ is preference regarding speaking $\mathrm{A}$ in private; $\pi_{b}$ is the preference regarding speaking $\mathrm{B}$ in private; $\xi$ is the conformity factor. . . . . . . . . . . . . . . . . . . . . . .

5.5 Schematic figure of the combined dynamics for the peer pressure model. Individuals choose their languages influenced by preferences in both public and private situations and receive pressure to conform in private according to the peer pressure model. $\lambda_{p u b}$ $\left(\lambda_{\text {priv }}\right)$ is the weight of public (private) interactions; $\pi_{A}\left(\pi_{a}\right)$ is preference regarding speaking A in public (in private); $\pi_{B}\left(\pi_{b}\right)$ is the preference regarding speaking $\mathrm{B}$ in public (in private); $\xi$ is the conformity factor. . . . . . . . . . . . 81

5.6 Schematic figure of the combined dynamics for the unanimity pressure model. Individuals choose their languages influenced by preferences in both public and private situations and receive pressure to conform in private according to the unanimity pressure model. $\lambda_{\text {pub }}\left(\lambda_{\text {priv }}\right)$ is the weight of public (private) interactions; $\pi_{A}\left(\pi_{a}\right)$ is preference regarding speaking $\mathrm{A}$ in public (in private); $\pi_{B}\left(\pi_{b}\right)$ is the preference regarding speaking $\mathrm{B}$ in public (in private); $\xi$ is the conformity factor. . . . . . . . . . 82

5.7 Equilibrium distribution of group types fractions in equilibrium for the peer pressure model with $n=3$ for different conformity levels $(\xi) . z_{i}$ corresponds to the fraction of the group type with $i A$ speakers. The dashed pink line represents the equilibrium fraction of $A$ speakers in the population, $z_{A}$. Private language dynamics with parameters: $\pi_{a}=0.5, \pi_{b}=0.6$.

5.8 Final fraction of $A$ speakers over $\pi_{a}$ values for the peer pressure model for fixed $\pi_{b}$. The red line (with $20 \%$ of transparency) corresponds to the case in which $\xi=1$, while the blue line (with $20 \%$ of transparency) depicts the situation when $\xi=100$. Private language dynamics with fixed parameter: $\pi_{b}=0.6 \ldots \ldots \ldots$. . . . 84

5.9 Trajectories of $z_{A}$ for different values of $\lambda_{p u b}$ using the peer pressure model. Parameters: $\pi_{A}=0.8, \pi_{B}=0.3, \pi_{a}=0.5, \pi_{b}=0.6, \xi=1$.

5.10 Critical values of $\lambda_{p u b}$ such that the final fraction of $z_{A}$ is larger or less than 0.5 for different levels of $\pi_{b}$ using the peer pressure model. Parameters: $\pi_{A}=0.8, \pi_{B}=0.3$, $\xi=1$ 
5.11 Equilibrium distribution of group types fractions in equilibrium for the unanimity pressure model with $n=3$ for different conformity levels $(\xi) . z_{i}$ corresponds to the fraction of the group type with $i A$ speakers. The dashed pink line represents the equilibrium fraction of $A$ speakers in the population, $z_{A}$. Private language dynamics with parameters: $\pi_{a}=0.5, \pi_{b}=0.6 \ldots \ldots \ldots \ldots \ldots$

5.12 Final fraction of $A$ speakers over $\pi_{a}$ values for the unanimity pressure model for fixed $\pi_{b}$. The red line (with $20 \%$ of transparency) corresponds to the case in which $\xi=1$, while the blue line (with $20 \%$ of transparency) depicts the situation when $\xi=10$. Private language dynamics with fixed parameter: $\pi_{b}=0.6 \ldots \ldots \ldots$. . . . . 86

5.13 Fractions of $A$ speakers over time for different initial conditions and different $\xi$ for the unanimity pressure model. The red lines represent the trajectories when the population is initially composed only by group types with only $A$ speakers. The blue lines represent the opposite situation, in which the population is initially composed of group types with only $B$ speakers. Solid lines and dashed lines correspond to the dynamics with $\xi=1$ and $\xi=100$, respectively. Private language dynamics with parameters: $\pi_{a}=0.5, \pi_{b}=0.6 \ldots \ldots \ldots \ldots \ldots \ldots$

5.14 Trajectories of $z_{A}$ for different values of $\lambda_{p u b}$ using the unanimity pressure model. Parameters: $\pi_{A}=0.8, \pi_{B}=0.3, \pi_{a}=0.5, \pi_{b}=0.6 \ldots \ldots \ldots$

5.15 Trajectories of $z_{0}, z_{1}, z_{2}$ and $z_{3}$ for different values of $\lambda_{\text {pub }}$ using the unanimity pressure model. Parameters: $\pi_{A}=0.8, \pi_{B}=0.3, \pi_{a}=0.5, \pi_{b}=0.6 \ldots \ldots \ldots$

5.16 Fractions of $A$ speakers over time for different initial conditions and different $\xi$ for the unanimity pressure model when $\lambda_{p u b}=0.4$ and $\lambda_{\text {priv }}=0.6$. The red lines represent the trajectories when the population is initially composed only by group types with only $A$ speakers. The blue lines represent the opposite situation, in which the population is initially composed of group types with only $B$ speakers. Solid lines and dashed lines correspond to the dynamics with $\xi=1$ and $\xi=10$, respectively. Other parameters: $\pi_{A}=0.6, \pi_{B}=0.5, \pi_{a}=0.2, \pi_{b}=0.2 \ldots \ldots \ldots \ldots$

5.17 Equilibrium points $z_{A}$ in function of $\pi_{A}$ for different $\lambda_{\text {pub }}$. Blue points represent the stable equilibrium points, while red crosses represent unstable equilibrium points or approximate saddle points. Parameters: $\pi_{B}=0.3, \pi_{a}=0.5, \pi_{b}=0.6, \xi=10 \ldots .91$ 
A.1 Necessary elements of a game: a set of strategies, number of players and a payoff function $f$. For illustrative purposes, here we limit $N=3$ players, we consider all the players have the same set of strategies and we show a map for only one strategy

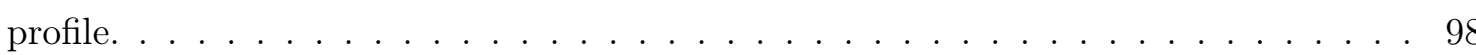




\section{Chapter 1}

\section{Introduction}

Although statistics has been used for some centuries to guide decisions on social problems, making mathematical models as an approach to understand social evolution is relatively new. It has joined efforts from biologists to economists and social scientists $[28,21,11,76]$, and there is no consolidated discipline that treats this approach exclusively ${ }^{1}$.

A badly digested doctrine of selfish genes [31] gave strength to ideas such as the character homo economicus, which is the basis of several mathematical models related to human behavior. As social beings, however, humans are not only motivated by individual benefits when they pursue their actions and, thus, explaining some behaviors based on the concept of the homo economicus has become a challenge.

The area of cultural evolution was born from this need of understanding more about social interactions and their effects at a societal level. Cultural evolution methods were derived from population biology and game theory, as its concern is to shed light on the changes which are products of the transmission of certain traits or behaviors.

In parallel, in Economics, the theory of social institutions was developed, also influenced by the ideas of evolutionary biology and game theory. An institution may be thought as the tool that governs social interactions [18]. Its evolution can trigger changes in individuals preferences and beliefs, and thus, the theory of social institutions is directly intertwined with the field of cultural evolution.

\footnotetext{
${ }^{1}$ Recently, a review on the emergence of consensus [13] has pointed out that two articles with very similar titles, The evolution of norms and The evolution of social norms, did not share a single bibliographic entry, showing how different fields do not interact with each other in the area of social evolution.
} 
One of the most studied problems of social evolution is the problem of the evolution of human cooperation. Cooperation can be considered the opposite behavior of selfishness, as it occurs when an individual benefits other individual without receiving any benefit from the recipient. Hamilton's rule [43] explains cooperation (among other phenomena) based on the relatedness between individuals.

Although Hamilton's rule is successful in explaining cooperation acts in dyadic interactions, still, this is not sufficient to understand large-scale cooperation, when individuals engage in non-dyadic interactions, as it is empirically and experimentally observed between humans. Cooperation seems to be imprinted in human behavior. And thus, studying this phenomena implies studying the process through which we came to be cooperative beings.

\subsection{Motivation and Main Results}

Cooperation underlies human social behavior, from the capability of joining efforts towards a common goal, to communication through the use of a language. In this thesis, we studied and created mathematical models for two different problems related to the field of evolution of cooperation: (i) evolution of altruism and (ii) language evolution.

\subsubsection{Evolution of altruism}

Previously, many studies have explained the evolution of altruism using several theories, from reciprocity and relatedness to multilevel selection. Fewer studies, however, have focused on the emergence of altruism, and how altruism can spread when altruists are rare in the population.

Schonmann, Caticha and Vicente [83] devised a new general framework (the two-level Fisher-Wright model) to analyze the evolution of social traits. Their model assumes a two-level selection: it considers the population is structured in groups of fixed size and that selection occurs within and between groups. Their main goal was to generalize Hamilton's rule, in order to account for the cases in which the fitness functions are non-linear, which cannot be treated by the generalized kin theory proposed by Taylor and Frank [96].

Inspired by the work of Samuel Bowles [16], we applied the two-level Fisher-Wright model to study the emergence of altruism in the presence of intergroup conflict. We extended the framework created 
by Schonmann, Caticha and Vicente [83], by incorporating war as an intergroup competition stage. We also built an agent-based simulation and compared its results with the results obtained with the theoretical model.

Our results show that it is possible that the occurrence of wars had influenced the spread of altruism in the population. We found that high frequencies of wars combined with large enough war benefits provided by altruists can make altruism viable even when migration levels are high (low relatedness). The agent-based simulation results agree with the theoretical model results.

\subsubsection{Language evolution}

The appearance of languages has been recognized as one of the major transitions in evolution. Language influences cultural evolution and, on the other hand, language diversity is a target of cultural evolution itself.

In the last century, language diversity has been steadily decreasing all over the world. This is a worrying situation, since there is no way to predict the effects of the extinction of so many languages. In particular, one common path for language extinction is the replacement of one language by a more powerful language. This is the case when dialects disappear after the introduction of a national standard language.

We studied the competition dynamics between a local dialect and a standard language. We created a mathematical model in which we assumed individuals could choose to use the local dialect or the standard language, according to different situations, public (e.g. workplace) and private (e.g. family meetings). We considered the choice of a dialect is mainly guided by two forces: (i) attractiveness of the local/standard language; and (ii) willingness to speak the same dialect (conformity factor) inside a private group.

Our results show that conformity usually enhances the effect dictated by the difference in attractiveness between the local dialect and the standard language if conformity works favoring only those states in which all speakers use the same dialect (unanimity pressure model). However, conformity may have no effect at all if it works in proportion to the fraction among peers (peer pressure model). These findings, when confronted with data from some dialects in Japan and in China, indicate that one way to preserve local dialects is to foster public policies focused in enhancing the pride of speaking local dialects. 


\subsection{Thesis Structure}

In this introduction, we discussed our motivations and provided a glimpse of the theory and the results we will present in the next chapters.

In chapter 2, we introduce the main mechanisms that promote the evolution and emergence of human cooperation. Part of this chapter is dedicated to multilevel selection, as this is a key concept to understand the models presented in chapter 3.

In chapter 3, we describe the core theory behind the framework Two-Level Fisher Wright (TLFW). TLFW is a general framework, which incorporates intra and intergroup selection and migration. In this chapter, we explain how this framework can model the evolution of social traits, when selection is observed at two different levels. We also describe how to calculate the conditions under which a certain social trait is viable and provide some examples of results obtained by the application of the framework to different public good games.

In chapter 4 , we introduce the model we created to investigate the emergence of altruism in the presence of intergroup conflict. We show how we adapted the TLFW framework to incorporate intergroup wars and present the results we obtained with the numerical simulations and the agentbased simulation.

In chapter 5 , we present our study of the survival of dialects when a more powerful language exists. We describe the motivations for creating this model and show which conditions may help a dialect to survive. A major part of this chapter is the result of a collaboration with Dr. Iwasa and Dr. Lee from Kyushu University, in Japan. Our work has been published in the journal Bulletin of Mathematical Biology [94].

In chapter 6 , we discuss how this thesis results contribute to the field and what are some possible future directions of the studied topics. 


\section{Chapter 2}

\section{Mechanisms for the evolution of}

\section{cooperation}

In the first edition of The selfish gene [31], Dawkins assumed individuals would all be "born selfish" and, thus, they should be taught altruism. Although, along the years, his opinion has changed, this idea of a innately selfish human reflected the strength, at the time, of the Homo Economicus character (the "rational" agent) in economics models.

However, many daily situations and laboratory experiments show that humans do not resemble the Homo Economicus character when making decisions. Cooperation levels are consistently higher than what it is expected from microeconomics theory.

This revealed to be a conundrum: how could we explain the observed cooperation levels from the interaction of self-interested individuals? Much of the theory behind the evolution of cooperation was developed as a response to the stated question, and different mechanisms through which cooperation could evolve were discovered (or rediscovered). Box 1 summarizes some of that mechanisms. 
Box 1: Examples of mechanisms for the evolution of cooperation.

The quest to understand human behavior regarding cooperation yielded simple rules which enabled self-regarding individuals to cooperate:

- kin relationships: sharing genes increase the likelihood of cooperation;

- reciprocity: the expectation of repeated interactions and the role of reputation inside a society can motivate behavioral changes towards more cooperative actions;

- sanctioning systems: reward or punishment, from an external authority or peers, can motivate individuals to cooperate.

As our understanding of humans as cooperative beings deepened, a myriad of psychological experiments challenged the proposed mechanisms, since humans seemed to be cooperative beyond kin relationships, the existence of sanctioning systems or the expectation of further interactions [19].

This chapter presents a concise introduction to the problems of evolution and emergence of cooperation. We describe the mechanisms of direct and indirect reciprocity and multilevel selection ${ }^{1}$ which help explaining how cooperation has evolved.

\subsection{The cooperation problem}

Before delving into the ways we can solve the cooperation problem, we should define more clearly what is the cooperation problem and how game theory can help us to elucidate it.

According to Hamilton [43], we can classify different social behavior from the fitness gains/losses faced by the actor and the recipient of a given action:

\begin{tabular}{|c|c|c|}
\hline & benefit to recipient & cost to recipient \\
\hline benefit to actor & mutualism & selfishness \\
\hline cost to actor & altruism & spite \\
\hline
\end{tabular}

Table 2.1: Categories of social behavior according to the interaction between actor and recipient.

In this thesis, we are mainly concerned with two concepts: altruism and cooperation. From table 2.1, altruism is the behavior in which an individual provides benefit to another individual at a cost to

\footnotetext{
${ }^{1}$ Although sanctioning systems are very important as a mechanism for the evolution of cooperation, for conciseness, we do not discuss it in this thesis. A good review of the main theory of sanctioning systems can be found in chapter 6 of $[90]$.
} 
himself. On the other hand, cooperation can be defined as a behavior in which an actor provides a benefit to a recipient, including both mutualistic and altruistic behaviors.

Although simple, these definitions have caused heated discussions and more than one author has attempted to provide a unified view of cooperation and/or altruism definitions [52, 105, 53]. One point of disagreement, for example, regards the way fitness effects should be accounted, as fitness effects might vary according to the time window, the target unit (population or group) etc., and this could potentially change what one views as altruism or spite [58]. In this chapter, we use cooperation and altruism interchangeably (unless specified), since we consider altruism is a restricted version of cooperation.

\subsubsection{Prisoner's Dilemma}

One of the most famous games in the field of game theory and studies about the evolution of cooperation is the Prisoner's Dilemma (PD) game. This game was proposed by scientists at RAND corporation, an American think tank, and has received its name when a RAND consultant, Albert Tucker described it as a situation in which two suspects are taken separately to be interrogated [77]. Each one of them faces the decision of choosing to betray the partner $(B)$, or to not betray him $(N)$, by remaining silent, knowing that according to the chosen action, they could be in jail for a very long, long or short time, or could be set free. One important feature here is that the players could not communicate with each other, so that in order to choose the best move, one has to make assumptions about how the other player will behave. This characteristic of the game is what distinguishes non-cooperative game theory from cooperative game theory, in which players can form coalitions among them.

A short introduction to game theory concepts and formalism can be found in appendix section A.1. In the two-person PD game, there are two players, the row player (player 1) and the column player (player 2). The set of strategies $S_{1}$, for player 1 , and $S_{2}$, for player 2 , are the same, such that: $S_{1}=S_{2}=\{B, N\}$, in which $B$ stands for Betray and $N$ stands for Not Betray. The rules in this game are as follows:

- if both players betray each other, they will only spend 1 year in prison each;

- if only one of them betrays the other, then the one who was betrayed will go to jail for 8 years while the other goes free; and 
- if both of them keep silent, they go to jail for 5 years.

This situation can be described by the following payoffs matrix:

$$
\begin{gathered}
N \\
N \\
B
\end{gathered}\left(\begin{array}{cc}
-1 & -8 \\
0 & -5
\end{array}\right)
$$

Note that the payoffs are only shown for one player, the row player, since the payoffs for the other player are reciprocal, i.e., the payoff for player 2 for the strategy profile $(B, N)$ is the payoff for player 1 when the strategy profile is $(N, B)$.

From eq. (2.1), we see that, if player 2 betrays player 1 , then, player 1 is better off by saying his partner committed the crime $(-5>-8)$. If player 2 remains silent, still, the best strategy is to betray him to go free $(0>-1)$. Since the scenario is exactly the same for player 2 , we observe that if both players are willing to choose the strategy that maximizes their own payoffs, they will end up choosing $B$, having to stand 5 years in prison. $(B, B)$ is called the Nash equilibrium of this game.

More generally, the PD game can be described by the following matrix [79, 98]:

$$
\begin{gathered}
C \\
C\left(\begin{array}{ll}
R & S \\
T & P
\end{array}\right)
\end{gathered}
$$

in which $T>R>P>S$. The letters $R, T, S$ and $P$ stand, respectively, for: Reward, Temptation, Sucker and Punishment.

In eq. (2.2), two strategies are available to the players: $C$ or $D$, respectively, to Cooperate (not betray) or to Defect (betray). Usually, it is also required that $2 R>T+P$, so that in one round, the reward payoff is higher than the average payoff of a defection - when considering the opponent is equally likely to play $C$ or $D$ with the same probability. The calculus of the Nash equilibrium follows the same steps taken before so that in the general form of the PD game, we find that $(D, D)$ is the strategy profile from which no player has incentive to deviate. 
Another interesting result associated with this game is its socially optimal strategy profile. A socially optimal strategy profile is the strategy profile which yields the highest sum of payoffs, considering all players. Let's assume $2 R>T+P$ in the general PD game with a payoff matrix as shown in eq. (2.2). Then, $(C, C)$ is the socially optimal strategy profile for the PD game, since $R>P$ and $2 R>T+P>T+S$. This means that the sum of the payoffs of players in the strategy profile $(C, C)$ is higher than the payoffs of players who chose $(C, D)$ or $(D, D)$, respectively. In other words, the socially best situation occurs when both players choose to cooperate in this game.

This mismatch between the socially optimal strategy profile and the Nash equilibrium is one of the reasons why the PD game has been used to tackle the problem of the evolution of cooperation. Because of this mismatch in the PD game, individuals have incentive to defect, instead of pursuing the prosocial behavior.

A second reason for using the PD game as a model game for studying cooperation is the possibility of capturing the essence of the so-called Tragedy of the Commons [44] through an $n$-person PD game (see section A.1.1): since it is always advantageous for an individual to overuse a public resource, the public resource would be depleted by its continuous overuse, affecting negatively all individuals.

\subsection{Kin selection}

When introducing natural selection theory, Darwin was concerned with the problem of self-sacrifice, which can be observed among bees, for example. Darwin had observed social insects in nature and had witnessed how many insects behave in a cooperative way, many times sacrificing their own reproductive chances to enhance the others' reproductive chances [33].

Although Darwin could explain this type of behavior as a consequence of the benefits it offered to kinship members, it was not until Hamilton's paper [43], in 1964, that this phenomena gained a mathematical form. Hamilton described a simple model for altruism, a special type of cooperation, in which an actor incurs a cost so that a recipient of the action can receive a benefit. This model was based on indirect fitness accounts into the actor's own fitness and the theory behind it has been called inclusive fitness, since it includes two types of fitness: direct and indirect fitness.

From Hamilton's model, a condition for the evolution of altruism is derived (Eq. 2.3). This condition involves the quantities $r, b$ and $c$, respectively, the relatedness between actor and recipient (see Box 2 ), the benefit for the recipient and the cost for the actor. It is important to notice that $b$ and $c$ 
are non-negative and their values can differ from the payoffs gained through interactions among the individuals.

$$
b r-c>0
$$

This simple rule helps explain many cases of altruism in nature. Since the rule discovery, empirical studies have estimated the values of $b$ and $c$ and many of them predicted with success which individuals were more likely to be a recipient for a cooperative action [15].

\footnotetext{
Box 2: What is $r$ ?

In textbooks [35], relatedness is usually defined as the probability that two individuals share the same allele. However, most of the time, this definition cannot be used to interpret $r$ in Hamilton's rule. In Hamilton's rule, $r$ is the chance of sharing the focal allele relative to the average probability that two individuals share the same allele, which is the allele's frequency in the population. Both of these definitions are the same when allele's frequency in the population is very low [15]. Relatedness is also defined as a regression coefficient, calculated from the regression of recipients' allele frequency on the actors' allele frequency. This is the formal way of calculating the relatedness as described by the definition above. Under this definition, random individuals are those whose relatedness is zero, i.e., the ones who share genes with each other with average frequency.
}

\subsection{Reciprocity}

Many of us have heard of the idiom: "you scratch my back and I'll scratch yours". Its meaning is clear: "if you help me, I will help you", the principle behind reciprocity.

Reciprocity can be distinguished into two different types: (i) direct reciprocity, and (ii) indirect reciprocity. While in direct reciprocity, individuals are directly benefited from their (past) behavior in a current game, in indirect reciprocity, the existence of a reputation system is responsible for publicizing the good behavior of a cooperator so that other individuals who never actually interacted with her may reciprocate. 


\subsubsection{Repeated games}

When we introduced the PD game before, we treated it as a one-shot game. However, it is not uncommon to play what is called repeated games, in which the same individuals repeatedly play the same game.

Two distinct approaches yield the same result:

- in microeconomic theory [40], repeated games are commonly explained in terms of discounted utility theory, according to which the payoff for each player is the sum of payoffs over the rounds weighted by a discount factor $\delta$, with $0<\delta<1$;

- in evolutionary game theory [90], on the other hand, $\delta$ is taken as the probability of playing another round of the game, so that, the probability that the game is played for exactly $n$ rounds is $\delta^{n-1}(1-\delta)$.

Note that when there is no uncertainty around the number of rounds, the last round of the finitely repeated PD game is equivalent to the one-shot $\mathrm{PD}$ game. This means that as in the one-shot PD game, we expect both players to defect, which is not socially optimal. Through backward induction, we can see that in the finitely repeated PD game, the strategy profile in which both players defect is the only subgame perfect Nash equilibrium.

In the infinitely repeated version of the PD game (or equivalently, a version in which players are not aware of when the game ends), within both approaches, the expected payoff (or the discounted utility) of one player is given by:

$$
V=v_{0}+\delta v_{1}+\delta^{2} v_{2}+\ldots+\delta^{n} v_{n}+\delta^{n+1} v_{n+1}+\ldots
$$

in which $v_{i}$ is the individual's payoff at the $i$-th round of the game.

Differently from the finitely repeated PD game, in this version, it is possible to achieve a state in which it is advantageous for both players to cooperate. As we shall see, this is a core property of the study of reciprocity. 


\subsubsection{Evolving direct reciprocity}

Early in 1970s, Trivers [98] proposed a model for the natural selection of what he called reciprocally altruistic behavior. He stated his motivation of studying this behavior with a simple example: if there is an individual drowning and someone not related or distantly related saves him, "[w]hy should the rescued individual bother to reciprocate? Selection would seem to favor being saved from drowning without endangering oneself by reciprocating. Why not cheat?".

In the same paper, after his own formulation of a model for the evolution of direct reciprocity, Trivers mentioned Hamilton had another formulation, which used game theory. Here we will use the game theoretical model, modified to account for the theory of repeated games presented in section 2.3.1.

One way to achieve the socially optimum configuration in the PD game is through the introduction of a strategy named tit-for-tat $(T F T)$ in which cooperation is used for as long as it takes the counterpart to defect, only cooperating again if the counterpart does so first. Note that a player that uses TFT can be considered to be a reciprocator, since his actions (with the exception of the first round) are merely a reflex of the other player's action in the previous round.

Suppose player 2 uses TFT whereas player 1 can choose between three strategies: AllC (playing $C$ in all rounds), AllD (playing $D$ in all rounds) and $T F T$. Also, let $V\left(s_{i} \mid s_{-i}\right)$ be the payoff of the player $i$ that plays strategy $s_{i}$ against the other player's strategy, $s_{-i}$.

At the start of the game, player 1 faces the following expected payoffs (following eq. (2.2)):

$$
\begin{aligned}
& V(\text { AllC } \mid \text { TFT })=R+\delta R+\delta^{2} R+\ldots+\delta^{n} R+\ldots=\sum_{k=0}^{\infty} \delta^{k} R \\
& V(T F T \mid T F T)=R+\delta R+\delta^{2} R+\ldots+\delta^{n} R+\ldots=\sum_{k=0}^{\infty} \delta^{k} R \\
& V(A l l D \mid T F T)=T+\delta P+\delta^{2} P+\ldots+\delta^{n} P+\ldots=T+\sum_{k=1}^{\infty} \delta^{k} P .
\end{aligned}
$$

From eq. (2.5), it is possible to see that there are only two possible outcomes:

- either both players cooperate indefinitely, if both players use strategy TFT or if one of them uses $A l l C$ and the other uses TFT; or

- one of them starts by cooperating but, after the defection from the AllD player, both of them defect indefinitely. This happens when one of the players uses AllD and the other uses TFT. 
Thus, $(C, C)$ will be achieved only if $V(A l l D \mid T F T)<V(T F T \mid T F T)=V(A l l C \mid T F T)$ or, equivalently, $T+\sum_{k=1}^{\infty} \delta^{k} P<\sum_{k=0}^{\infty} \delta^{k} R$.

This condition can be calculated explicitly in function of $R, T$ and $P$ :

$$
\begin{aligned}
& V(\text { AllD } \mid T F T)<V(T F T \mid T F T) \Longrightarrow T+\sum_{k=1}^{\infty} \delta^{k} P<\sum_{k=0}^{\infty} \delta^{k} R \\
& \Longrightarrow T+\delta P \sum_{k=0}^{\infty} \delta^{k}<\sum_{k=0}^{\infty} \delta^{k} R \Longrightarrow T+\frac{\delta P}{1-\delta}<\frac{R}{1-\delta} \\
& \Longrightarrow \delta>\frac{T-R}{T-P} .
\end{aligned}
$$

Since $P<R$ in a prisoner's dilemma game, then, $T-P>T-R \Leftrightarrow 0<\frac{T-R}{T-P}<1$, i.e., it is always possible to have a sufficient high $\delta$ that makes cooperation evolve when one of the players is a reciprocator (i.e. uses $T F T$ ). The interpretation is that it is necessary a sufficient high discount rate or a sufficient high number of rounds for cooperation to be more advantageous than defection in the infinitely repeated prisoner's dilemma when one of the players uses TFT.

Another way to analyze this model is to use the concept of evolutionary stable strategies (ESS), as proposed by Maynard Smith and George Price [92] in the context of computer simulations. In fact, in the previous calculation, we found the condition for $V(A l l D \mid T F T)<V(T F T \mid T F T)$, i.e., the condition for TFT to be an ESS against AllD (more details about the ESS definition can be found in the appendix section A.2).

In 1978, a political scientist, Axelrod, used the same type of simulation for analyzing different strategies performances in prisoner's dilemma games [11]. In his setting, in each round, individuals were paired and played a PD game. Each individual played the game using one strategy, e.g. AllC, AllD etc.

The main objective of Axelrod was to compare several strategies and find the winning strategy, i.e., the one that achieved the highest average payoff. In the first tournament promoted by Axelrod, fourteen strategies were subscribed. The winner was the simple strategy tit-for-tat (suggested by Rapoport), which cooperates on the first round and then copies the behavior of the opponent for the next rounds (the same as the TFT strategy introduced previously). Indeed, it was a very robust strategy, since it also won the second tournament promoted by Axelrod, in which sixty strategies competed against each other. 
Although TFT is an ESS against AllD, it is easy to see that it is not an unbeatable strategy in the evolutionary sense: since one cannot distinguish AllC and TFT when playing against each other, it is possible that $A l l C$ increases by random drift and, if it eliminates $T F T$, it would, subsequently, be invaded by $A l l D$. In fact, when no mistakes are allowed, there are no evolutionary stable pure [20] or mixed strategies [34] in the repeated prisoner's dilemma game.

Only in the beginning of 1990s, Nowak and Sigmund [69] rediscovered the strategy called Simpleton (proposed by Rapoport), also known as Pavlov, and realized it could generate even higher payoffs than tit-for-tat. The Pavlov strategy uses the reasoning "win-stay, lose-shift": if it receives a high payoff ( $R$ or $T$ ), it remains using the same behavior; however, if it receives a low payoff ( $P$ or $S)$, it changes the behavior.

The key to the success of Pavlov strategy is the allowance of mistakes when implementing the strategies. Thus, for instance, instead of changing from $C$ to $D$ with probability 1 when getting the payoff $S$, one individual playing Pavlov will make this change with probability $1-\epsilon$, with $0<\epsilon<1$. This translates into a chance of changing from $C$ to $D$, even when getting $R$, which can be advantageous if Pavlov is playing against an $A l l C$ strategy (because $A l l C$ will not defect, by changing to $D$, Pavlov will retain the highest payoff, $T$, for all subsequent rounds).

\subsubsection{Evolving indirect reciprocity}

Today, especially with the aid of Internet, a considerable amount of human daily interactions occur between strangers. Direct reciprocity cannot explain cooperation in these cases, since in this type of interaction, the likelihood of further encounters with the same individual is very low.

Indirect reciprocity, on the other hand, may explain how humans sustain cooperation even in this setting: the principle is that an individual will cooperate if somebody else has cooperated with him before. One way to conceive this act is to think individuals are prone to help when they had a recent experience of being helped before. This has been termed upstream reciprocity (or misguided reciprocation [90]) and, in daily life, it can be seen, for example, in "pay it forward" campaigns ${ }^{2}$, in which one customer is offered the opportunity to leave a product paid to the next customer who enters the store.

\footnotetext{
${ }^{2}$ For instance, Starbucks reported that 19,000 customers in South China bought coffee for the next customer in a pay it forward campaign in 2014 [1]. Although there were some breaks in the generosity chain, in which Starbucks intervened, by providing the coffee for the next customer, there seems to be a social contagion effect in place.
} 
When the population is large, Nowak and Roch [72] showed that upstream reciprocity may foster the evolution of cooperation when combined with other mechanisms, such as direct and spatial reciprocity. This result is consistent with previous results obtained by Boyd and Richerson [23], which compared a TFT based on "be nice to people who are nice to others" (termed downstream reciprocity or vicarious reciprocation [90]) with a TFT based on "be nice if others are nice to you" (upstream reciprocity), finding that the former seemed to be more successful in promoting cooperation for groups with a larger number of players.

Hence, in the remainder of this section, we focus on downstream reciprocity, and introduce important results from reputation-based indirect reciprocity models. One difference from direct reciprocity models is that most models of indirect reciprocity, are built from a donor game, instead of using the traditional prisoner's dilemma game.

In the donor game, two roles exist: donor or recipient. While as a donor, an individual is able to choose between helping the recipient (by incurring a cost $c>0$ ) or not, as a recipient, an individual merely accepts being helped (receiving a benefit $b$ ) or not being helped. This game, thus, is a degenerate game, i.e., only one of the players (the donor) has a set with more than one strategy to choose from. Note that two rounds of the game with an individual placed as a donor in one round and as a recipient in another round yields the same payoffs as the original two-person prisoner's dilemma game.

Another notion that is inexistent in direct reciprocity models is the concept of a score for players. This score can be binary (good or bad) or a "full score", and intends to classify individuals according to their cooperativeness. This means individuals would be able to build a reputation by taking actions which preserve or lead to a "good" score.

The simplest model of indirect reciprocity considers an infinite population, with the same assumptions of models of direct reciprocity: probability $0<w<1$ of having another round; and interaction between pairs of individuals through a PD game [73]. There are two strategies in the model: DSC (discriminator) and AllD. While individuals playing $D S C$ only cooperate with those who cooperated in the previous round, individuals who use $A l l D$ always defect. In this situation, $D S C$ yields the same payoff as a TFT strategy, and hence is an ESS, i.e., it cannot be invaded by AllD.

When thinking about what determines an individual's reputation, the following question arises: should a good player become a bad player if he refused help to a bad player? Also, it is not clear how individuals actions depend on their own reputation and on the partner's reputation. Ohtsuki 


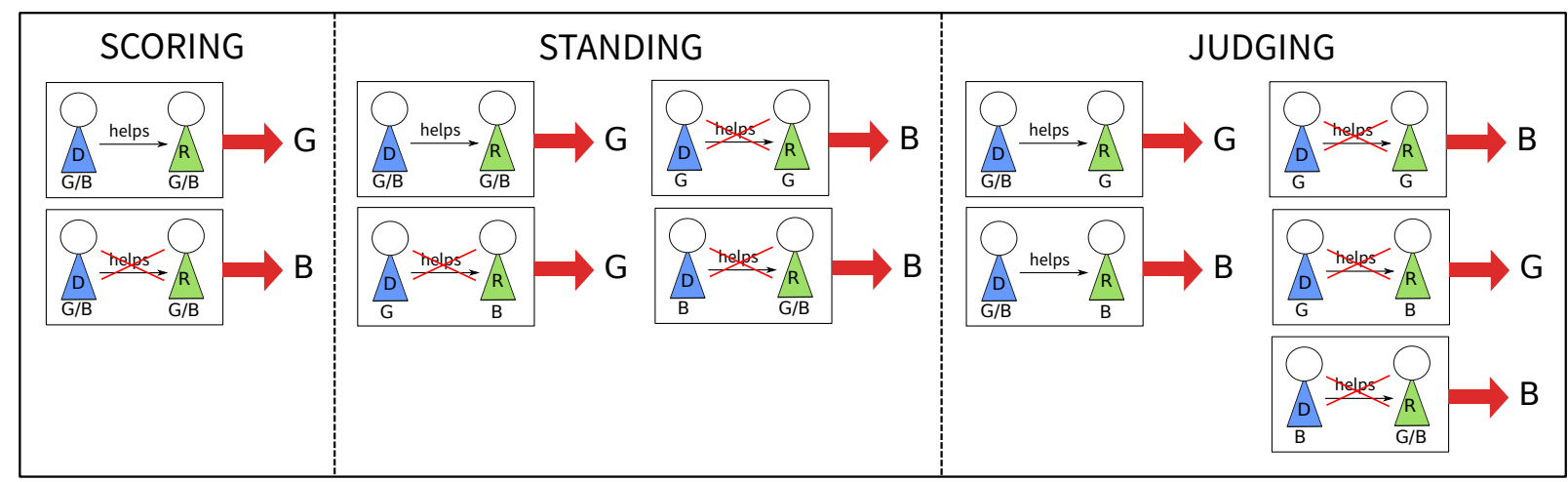

Figure 2.1: Examples of assessment rules. In SCORING, reputations do not matter, and a donor $(D)$ who helps a recipient $(R)$ is always labeled $\operatorname{Good}(G)$, while donors who do not help recipients are always labeled $B a d(B)$. In STANDING, the difference from the SCORING rule is that a $G$ donor who refuses to help a $B$ recipient is still considered to be $G$. In JUDGING, the difference from the STANDING rule is that helping a $B$ recipient makes a donor to be labeled as $B$.

and Iwasa [74] developed a model that took into account several assessment rules (how users score other users) and action rules (how donors choose whether to help or not a recipient), and calculated the evolutionary stability of different strategies, which combined assessment rules with action rules.

In their model, they consider a binary score system, in which individuals can be labeled as Good $(G)$ or Bad $(B)$. All individuals are born Good and are assessed according to the last played game as a donor. Assessment rules determine what is the reputation of the donor according to his choice of helping or not a recipient in all possible combinations of donor-recipient reputations, $G$ or $B$. Some examples of assessment rules can be seen in fig. 2.1.

By assessing other individuals, when in the role of a donor, an individual is able to choose his own actions based on the reputation of the recipient (and even looking at his own reputation). Action rules determine whether the donor will help or not help a recipient for all types of situations. The most extreme action rules are $A l l C$ which makes a donor help all recipients; and $A l l D$ which makes a donor refuses to help any recipient. Other action rules are shown in fig. 2.2.

Since both sets of rules (assessment and action) are used to compose a strategy, the total number of strategies is very large: $2^{8} \times 2^{4}=2^{12}=4096$ different strategies. Note that, while all action rules were covered by the set of $A l l C$, AllD and the action rules in fig. 2.2, there are many more assessment rules than what was covered in fig. 2.1. 


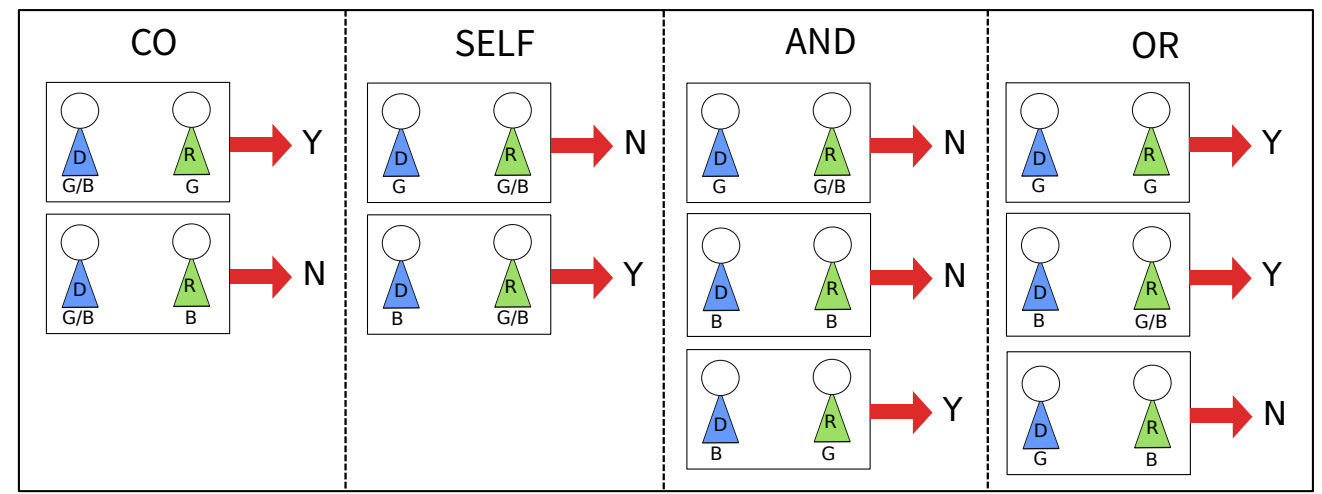

Figure 2.2: Examples of action rules. In $\mathrm{CO}$, only the reputation of the recipient $(R)$ is used to make the decision of helping $(Y)$ or not $(N)$ an individual: if the recipient is Good $(G)$, the donor $(D)$ helps him, otherwise, if the recipient is $B a d(B)$, the donor will refuse help to him. In SELF, the decision is based solely on the reputation of the donor: a donor helps a recipient only if his own reputation is $B$. In AND, a donor helps a recipient only if his own reputation is $B$ and if the reputation of the recipient is $G$. In OR, a donor helps a recipient if his own reputation is $B$ or if the reputation of the recipient is $G$.

Iwasa and Ohtsuki investigated whether a population composed by a majority of individuals playing one strategy (for instance, CO-STANDING) could be invaded by a small number of individuals playing another strategy (e.g., AND-STANDING). They considered implementation errors and assessment errors, along with different parameter values of benefit $(b)$ and cost $(c)$. Since the number of strategies was very large, in order to be able to make the analytical calculations, Iwasa and Ohtsuki assumed a very large population and only compared strategies within the same assessment module. Also, they assumed a public scoring, so that any individual had the same view of reputation labels for the other individuals.

After considering all possible pairs of strategies, they reached a core with eight strategies which were robust to invasion, even considering a wide range of probability of assessment and implementation errors and values of $c$ slightly larger than $b$ [25]. This group of ESS strategies was called the leading eight(see table 2.2).

We can see that for the action modules, only CO and OR appear in the table with leading eight strategies. Note that the leading strategy number 1 is CO-STANDING, while the leading strategy number 8 is CO-JUDGING. All the other leading strategies have assessment modules which are not represented in fig. 2.1. Simple rules are prevalent, though: (i) helping a good recipient always yields a good reputation; (ii) refusing to help a good recipient always yields a bad reputation; and (iii) refusing to help a bad recipient yields a good reputation. 


\begin{tabular}{ccccccccc}
\hline situation/strategy & 1 & 2 & 3 & 4 & 5 & 6 & 7 & 8 \\
\hline$G \rightarrow G$ & $G$ & $G$ & $G$ & $G$ & $G$ & $G$ & $G$ & $G$ \\
$G \rightarrow B$ & $G$ & $B$ & $G$ & $G$ & $B$ & $B$ & $G$ & $B$ \\
$B \rightarrow G$ & $G$ & $G$ & $G$ & $G$ & $G$ & $G$ & $G$ & $G$ \\
$B \rightarrow B$ & $G$ & $G$ & $G$ & $B$ & $G$ & $B$ & $B$ & $B$ \\
$G \nrightarrow G$ & $B$ & $B$ & $B$ & $B$ & $B$ & $B$ & $B$ & $B$ \\
$G \nrightarrow B$ & $G$ & $G$ & $G$ & $G$ & $G$ & $G$ & $G$ & $G$ \\
$B \nrightarrow G$ & $B$ & $B$ & $B$ & $B$ & $B$ & $B$ & $B$ & $B$ \\
$B \nrightarrow B$ & $B$ & $B$ & $G$ & $G$ & $G$ & $G$ & $B$ & $B$ \\
$G \stackrel{?}{\rightarrow} G$ & $Y$ & $Y$ & $Y$ & $Y$ & $Y$ & $Y$ & $Y$ & $Y$ \\
$G \stackrel{?}{\rightarrow} B$ & $N$ & $N$ & $N$ & $N$ & $N$ & $N$ & $N$ & $N$ \\
$B \stackrel{?}{\rightarrow} G$ & $Y$ & $Y$ & $Y$ & $Y$ & $Y$ & $Y$ & $Y$ & $Y$ \\
$B ? \rightarrow ?$ & $Y$ & $Y$ & $N$ & $N$ & $N$ & $N$ & $N$ & $N$ \\
\hline
\end{tabular}

Table 2.2: The leading eight strategies. For each strategy (columns numbered from 1 to 8), both assessment rules (first 8 rows) and action rules (last 4 rows) are shown. Assessment rules situations are represented showing the reputation of the donor (Good, $G$, or Bad, B), an arrow which may indicate whether the donor helped $(\rightarrow)$ or not $(\nrightarrow)$ the recipient, and the reputation of the recipient $(G$ or $B)$. Action rules are represented showing the reputation of the donor $(G$ or $B)$, an arrow $\stackrel{?}{\rightarrow}$ and the reputation of the recipient; possible actions are $Y$ (to help) or $N$ (to refuse to help).

\subsection{Multilevel selection}

Through the study of natural selection, we can see Darwin's principles could apply to any entity among different levels of organization in a species. Multilevel selection, also known as group(-trait) selection or population selection, refers to simultaneous selection processes that occur at different hierarchical levels, e.g. individual and group of individuals [75]. Its multiple denominations are part of its controversies among researchers in evolutionary biology field.

The general feeling about multilevel selection has changed during 20th century from acceptance to rejection and, then, a revival of the theme in recent years. While in the beginning of 20th century, Darwin's arguments about the relation between group selection and social behavior were relatively well accepted, later publications shifted the acceptance to an almost complete rejection of multilevel selection for many years [14]: 
The theory of group selection has been so unpopular for the past 40 years that, until recently, it existed in the scientific limbo, somewhere between being a heretical curiosity and a useless dead end.

The past 40 years mentioned by Borello refers to the period starting in 1960s, and Wilson [106] explains the motivations behind it:

The rejection of group selection was based on three arguments. First, theoretical models indicated that D. S. Wilson and E. O. Wilson between-group selection is a weak evolutionary force compared to within-group selection. Second, the empirical evidence for group selection was speculative and could just as easily be explained in terms of within-group selection. Third, the alternative theories seemed to provide more robust explanations of altruism and cooperation without invoking group selection.

Although this idea was widespread in the community of evolutionary biologists, there was still doubt regarding the role of multilevel selection at evolutionary processes. In his review of the existing group selection models, Wade [102] first presented some inconsistencies in the terminology of multilevel selection among different researchers and, then, provided a thorough review of the compounding elements of group selection models.

A decade later, Mayo and Gilinski [61] proposed that group-level fitness could be classified in two different ways. Group-individual fitness refers to the average fitness of individuals in one group. On the other hand, group-group fitness refers to the number of groups produced, on average.

According to them, this distinction separates the models of group selection into two different types: type I models, related to group-individual fitness; and type II models, related to group-group fitness. While type I models are concerned with differences among groups due to distinct individual rates of production, type II models focus on differences among groups due to different production rates of new groups. In their paper, they provide an example that elucidates the need for these two core concepts [61]:

Suppose that the average fitness of flightless beetle individuals is $\mathrm{k}$, and the average fitness of flying beetle individuals is $m$, where $m$ exceeds $k$. Then the group-individual fitnesses of the group of flightless and the group of flying beetles are $k$ and $m$, respectively, and the two groups differ in group-individual fitness. Suppose, however, that 
the group of flightless beetles produces 100 distinct new groups of flightless beetles on average, and the group of flying beetles produces only 10 distinct groups of flying beetles, on average. Then the group-group fitnesses of the group of flightless and the group of flying beetles are 100 and 10, respectively, and the two groups differ in group-group fitness. While the group of flightless beetles is less fit than the group of flying beetles from the perspective of group-individual fitness, the group of flightless beetles is more fit than the group of flying beetles from the perspective of group-group fitness.

They argue that although Type I models are of interest as a way to view the evolutionary process, these models do not identify a causal group-level selection process, different from individual selection. In their opinion, only Type II models can explain differences among groups that are due to exclusively group-level events.

This distinction in multilevel selection (MLS) types was also pointed out by Damuth and Heisler [30] and Okasha [75]. However, they use a different terminology: while MLS $\mathrm{S}_{1}$ is concerned with the change in the frequencies of individuals, $\mathrm{MLS}_{2}$ describes this change for different types of groups.

According to Okasha [75], in order to study multilevel selection, we have to define the mechanisms required by selection (character variation, fitness differences and heritability) at the multiple levels in question. He distinguishes two methods for calculating the group-level fitness: collective fitness 1 , which counts the number of daughter individuals, and collective fitness 2 , which counts the number of daughter groups. In models that allow different group sizes, this is an important issue, since it determines whether a group giving birth to 4 groups of 3 individuals is more successful (or not) than a group that gives birth to 3 groups of 4 individuals each.

Although there are still some disagreement around the concept of multilevel selection, the current situation is much more favorable to it, as Wilson summarizes [106]:

Today, after four decades of research, it has become clear that the 1960s consensus was in error. Group selection is theoretically plausible, there is solid empirical evidence for it, and the alternative theories have multilevel selection embedded within their own structures.

In the next paragraphs, we present the Price equation, which has been used in both kin selection and multilevel selection models. We also derive the Hamilton's rule from the Price equation, as 
the derivation is helpful to understand its limitations as a rule to predict cooperation between individuals.

\subsubsection{The Price equation}

The Price equation has been extensively used for modeling social evolution. This equation [78] expresses the difference of the frequency of a type of particle or individual between two subsequent generations. It is simple, recursive and separates clearly the effects of inter and intragroup selection [42].

Different from other models, however, the Price equation was not developed with the intention to model one specific natural phenomenum. Hence, it does not rely on simplified assumptions about reality. It is rather built in an abstract way so that it fits several biological situations.

In the original paper [78] that derived the equation, two populations $\psi_{1}$ and $\psi_{2}$ are considered. $\psi_{1}$ is the population consisting of the parents of $\psi_{2}$, so that $\psi_{2}$ members are offspring of $\psi_{1}$. Population members of $\psi_{1}$ are arbitrarily labeled as $1,2, \ldots, \mathrm{N}$ (total population size). The main objective is to track one character (e.g., gene A) frequency in these two generations. This derivation uses many symbols; these symbols and their interpretations can be seen in table 2.3.

\begin{tabular}{|c|l|}
\hline Symbol & Meaning \\
\hline$N$ & population size of $\psi_{1}$ \\
$n_{z}$ & zygotic ploidy \\
$g_{i}$ & dose of gene A in individual $i$ (if $n_{z}=2$, then, $g_{i}$ can be 0,1, or 2.) \\
$p_{i}$ & frequency of gene $A$ in individual $i, p_{i}=g_{i} / n_{z}$ \\
$P_{1}$ & frequency of gene $A$ in population $\psi_{1}$ \\
$n_{G}$ & gametic ploidy \\
$w_{i}$ & number of offspring produced (successful gametes) by individual $i$ \\
$g_{i}^{\prime}$ & number of genes $A$ in the set of successful gametes from individual $i$ \\
$p_{i}^{\prime}$ & frequency of gene $A$ in $i$ 's set of successful gametes, $p_{i}^{\prime}=g_{i}^{\prime} / w_{i} n_{G}$ \\
$P_{2}$ & frequency of gene $A$ in population $\psi_{2}$ \\
$\Delta p_{i}$ & difference in frequency of gene A between $i$ and her offspring, $p_{i}^{\prime}-p_{i}$ \\
\hline
\end{tabular}

Table 2.3: Symbols used in Price equation derivation. 
To find the difference in frequency of gene A between the two generations represented by population $\psi_{1}$ and $\psi_{2}$, it is necessary to calculate $P_{1}$ and $P_{2}$. Note that both of these quantities are just the sum of the individual frequencies of all individuals in populations $\psi_{1}$ and $\psi_{2}$ divided by the population size. In the case of $\psi_{1}$, the calculation is straightforward, as we can see in eq. (2.7). For individuals of population $\psi_{2}$, we calculate $P_{2}$ from the sum of total frequencies of all offspring for each individual $i$, which is simply $z_{i} p_{i}^{\prime}$.

$$
\begin{gathered}
P_{1}=\frac{\sum_{i} p_{i}}{N}=\bar{p} \\
P_{2}=\frac{\sum_{i} w_{i} p_{i}^{\prime}}{\sum_{i} w_{i}}=\frac{\sum_{i} w_{i} p_{i}^{\prime}}{N \sum_{i}\left(w_{i} / N\right)} \\
=\frac{\sum_{i} w_{i} p_{i}^{\prime}}{N \bar{w}}=\frac{\sum_{i} w_{i}\left(p_{i}+\Delta p_{i}\right)}{N \bar{w}} \\
=\frac{\sum_{i} w_{i} p_{i}}{N \bar{w}}+\frac{\sum_{i} w_{i} \Delta p_{i}}{N \bar{w}} \\
=\frac{\left(\bar{w} \bar{p}+\operatorname{Cov}\left(w_{i}, p_{i}\right)\right)}{\bar{w}}+\frac{\sum_{i} w_{i} \Delta p_{i}}{N \bar{w}}
\end{gathered}
$$

in which $\bar{w}=\sum_{i} w_{i} / N$ is the average number of individuals of offspring produced in the population and, in the last equality, we use the formula for the covariance between two variables $x=\left(x_{1}, \ldots, x_{n}\right)$ and $y=\left(y_{1}, \ldots, y_{n}\right)$, given by $\operatorname{Cov}\left(x_{i}, y_{i}\right)=\frac{\sum_{i} x_{i} y_{i}}{n}-\left(\frac{\sum_{i} x_{i}}{n}\right)\left(\frac{\sum_{i} y_{i}}{n}\right)$.

Using $P_{1}$ and $P_{2}$ as obtained in eqs. (2.7) and (2.8), the Price equation is derived by calculating the quantity $\Delta P=P_{2}-P_{1}$ in the following way:

$$
\begin{aligned}
\Delta P & =\left(\bar{p}+\frac{\operatorname{Cov}\left(w_{i}, p_{i}\right)}{\bar{w}}+\frac{\sum_{i} w_{i} \Delta p_{i}}{N \bar{w}}\right)-\bar{p} \\
& =\frac{\operatorname{Cov}\left(w_{i}, p_{i}\right)}{\bar{w}}+\frac{\mathrm{E}\left(w_{i} \Delta p_{i}\right)}{\bar{w}}
\end{aligned}
$$

in which we replaced $E\left(w_{i} \Delta p_{i}\right)=\frac{\sum_{i} w_{i} \Delta p_{i}}{N}$.

Usually, the Price equation is written in another form eq. (2.10), using a slight different notation and interpretation from what we introduced before: $w$ represents fitness (relative or absolute, since fitness scaling does not alter the equation), $z$ represents the character value (also called phenotype, when it is defined as a mathematical function of traits) and $z_{i}$ represents the frequency of the character value in individual $i$.

$$
\Delta \bar{z}=\frac{\operatorname{Cov}\left(w_{i}, z_{i}\right)}{\bar{w}}+\frac{\mathrm{E}\left(w_{i} \Delta z_{i}\right)}{\bar{w}}
$$


This equation has two terms on the right side, which are, respectively, referred to be related to selection and transmission processes [36, 81]. The covariance term represents the change of the character in the population due to differential fitness and reproduction. If larger character values increase fitness, the covariance is positive and it will increase the population average character value. The remainder term in the right side encompasses the processes involved in the transmission of the character, like mutation or recombination. It represents how much the offspring resemble their parents. If offspring are exact copies of their parents, the right term is zero.

\subsubsection{From Price equation to Hamilton's rule}

One way to derive Hamilton's rule is to consider Price equation along with a few number of assumptions. These assumptions clarify the relationship between Hamilton's rule and linear fitness functions, which is crucial to understand the motivations of the framework we present in the next chapter. Here we follow the derivation described in [63].

Firstly, we assume individuals are perfect copies from their parents, so that the frequency of gene $A$ does not vary across generations, i.e., $\Delta p_{i}=0$. From eqs. (2.7) and (2.8), and using one of the intermediate formulas of $P_{2}$, we can rewrite the difference $\Delta P=P_{2}-P_{1}$ as the following equation:

$$
\begin{aligned}
\Delta P & =\frac{\sum_{i} w_{i} p_{i}}{N \bar{w}}-\frac{\sum_{i} p_{i}}{N} \\
& =\frac{\mathrm{E}\left(w_{i} p_{i}\right)}{\bar{w}}-\frac{\mathrm{E}\left(w_{i}\right) \mathrm{E}\left(p_{i}\right)}{\bar{w}} .
\end{aligned}
$$

The version of Price equation given by eq. (2.11) can be further simplified into:

$$
\bar{w} \Delta P=\operatorname{Cov}\left(w_{i}, p_{i}\right) .
$$

Secondly, we should define a general equation for the fitness function $w_{i}$ of individual $i$. We can do that by assuming additive fitness effects, which means gain/loss in fitness is proportional to the probability of the particular event which led to that gain/loss. Letting $b>0$ be the gain in fitness obtained by the occurrence of an event of probability $y_{i} ; c>0$ be the loss in fitness obtained by the occurrence of an event of probability $h_{i}$; and $w_{0} \geq 0$, constant, be the baseline fitness of an individual $i$, then, we have the following fitness function for individual $i$ :

$$
w_{i}=w_{0}+y_{i} b-h_{i} c .
$$


Since our main goal is to derive Hamilton's rule, in the next paragraphs, we restrict our analysis to the case when gene $A$ is related to altruism. In the specific case of altruism, eq. (2.13) implicates that being helped more increases $w_{i}$ linearly with a factor $b$ and that helping the others more decreases $w_{i}$ linearly with a factor $-c$.

Thirdly, since eq. (2.13) involves two more terms $y_{i}$ and $h_{i}$, we should specify their general form for being able to calculate eq. (2.12) with $w_{i}$ as given by eq. (2.13). This is done by assuming additive genetics, which implies that the behavior of an individual depends on the genotype of the actor or the recipient of altruism. This means $y_{i}$ and $h_{i}$ should be written as linear functions of, respectively, $p_{j}$ and $p_{i}$, the frequency of gene $A$ in the recipient of altruism $j$ and his own frequency of gene $A$ (when he is the actor):

$$
\begin{gathered}
y_{i}=a+k p_{j} \\
h_{i}=a+k p_{i},
\end{gathered}
$$

in which $a$ and $k$ are parameters of the linear function and are chosen so that $0 \leq y_{i} \leq 1$ and $0 \leq h_{i} \leq 1$.

With these definitions, we can finally use $w_{i}$ from eq. (2.13) and $y_{i}$ and $h_{i}$ from eq. (2.14) to calculate eq. (2.12):

$$
\begin{aligned}
\bar{w} \Delta p & =\operatorname{Cov}\left(w_{0}+y_{i} b-h_{i} c, p_{i}\right) \\
& =\operatorname{Cov}\left[w_{0}+\left(a+k p_{j}\right) b-\left(a+k p_{i}\right) c, p_{i}\right] \\
& =\operatorname{Cov}\left(w_{0}, p_{i}\right)+\operatorname{Cov}\left(a b, p_{i}\right)+\operatorname{Cov}\left(b k p_{j}, p_{i}\right)-\operatorname{Cov}\left(a c, p_{i}\right)-\operatorname{Cov}\left(c k p_{i}, p_{i}\right) .
\end{aligned}
$$

Many of these covariance terms disappear since one of the variables is actually a constant, e.g. $\operatorname{Cov}\left(w_{0}, p_{i}\right)$, yielding:

$$
\bar{w} \Delta p=b k \operatorname{Cov}\left(p_{j}, p_{i}\right)-c k \operatorname{Var}\left(p_{i}\right)
$$

This means that the condition for the survival of the altruist gene, $\Delta p>0$, implies:

$$
b\left(\frac{\operatorname{Cov}\left(p_{j}, p_{i}\right)}{\operatorname{Var}\left(p_{i}\right)}\right)-c>0 .
$$


Note that $\operatorname{Cov}(x, y)=\operatorname{Var}(x) \beta(y, x)$, in which $\beta(y, x)$ is the slope of the regression line of $y$ on $x$, so that eq. (2.17) can be written as:

$$
b \beta\left(p_{j}, p_{i}\right)-c>0 .
$$

The condition for the survival of the altruist gene as written in eq. (2.18) is already close to Hamilton's rule. What remains to be done is to link the regression coefficient, $\beta\left(p_{j}, p_{i}\right)$, to relatedness, $r$. In order to this, we need an additional assumption: absence of selection for altruism, which means the allele for altruism is as likely to be selected as any other allele. This assumption provide us a way of calculating $r$, which can be defined as the fraction of genes IBD (identical by descent) in two individuals, $i$ and $j$.

Let $p_{j, \mathrm{IBD}}$ be the average proportion of altruism genes relative to the number of genes which are IBD in individuals $i$ and $j$. Also, let $p_{j, \overline{\mathrm{BD}}}$ be the the average proportion of altruism genes relative to the number of genes which are not IBD in individuals $i$ and $j$. Then, the expected frequency of gene $A$ for individual $j$ given the frequency of gene $A$ for individual $i, \mathrm{E}\left(p_{j} \mid p_{i}\right)$, is given by:

$$
\mathrm{E}\left(p_{j} \mid p_{i}\right)=r p_{j, \mathrm{IBD}}+(1-r) p_{j, \overline{\mathrm{IBD}}}
$$

Note that $p_{i}$ is the frequency of gene $A$ for individual $i$, which means, on average, $p_{i}$ of the IBD genes of individuals $i$ and $j$ are altruism genes, since there is no selection for any particular gene, so that $p_{j, \mathrm{IBD}}=p_{i}$. On the other hand, on average, the proportion of non-IBD genes of individuals $i$ and $j$ which are altruism genes, $p_{j, \overline{\mathrm{IBD}}}$, is simply the frequency of gene $A$ in the population, $p=\sum_{i} p_{i} / N$. Thus:

$$
\mathrm{E}\left(p_{j} \mid p_{i}\right)=r p_{i}+(1-r) p_{j}
$$

Another way to calculate $\mathrm{E}\left(p_{j} \mid p_{i}\right)$ is to use the coefficients $\alpha$ and $\beta\left(p_{j}, p_{i}\right)$ of a first order linear regression model:

$$
\mathrm{E}\left(p_{j} \mid p_{i}\right)=\alpha+\beta\left(p_{j}, p_{i}\right) p_{i}
$$

In eq. (2.21), we should find the intercept $\alpha$ in order to calculate $\mathrm{E}\left(p_{j} \mid p_{i}\right)$. This can be done by calculating least-squares estimate of the intercept of $\mathrm{E}\left(p_{j} \mid p_{i}\right)$ or, in a intuitive way, we can take the expectation of both sides of eq. (2.21), so that $\alpha=\mathrm{E}\left[\mathrm{E}\left(p_{j} \mid p_{i}\right)\right]-\beta\left(p_{j} \mid p_{i}\right) \mathrm{E}\left(p_{i}\right)$, which implies that 
$\alpha=\mathrm{E}\left(p_{j}\right)-\beta\left(p_{j}, p_{i}\right) \mathrm{E}\left(p_{i}\right)$. Replacing $\alpha$ in eq. (2.21), we have:

$$
\mathrm{E}\left(p_{j} \mid p_{i}\right)=\left(1-\beta\left(p_{j}, p_{i}\right)\right) \mathrm{E}\left(p_{i}\right)+\beta\left(p_{j}, p_{i}\right) p_{i}
$$

in which we used the fact that $\mathrm{E}\left(p_{j}\right)=\mathrm{E}\left(p_{i}\right)$, which is valid for any $i$ and $j$.

Hence, by inspection of eqs. (2.20) and (2.22), we can see that $r=\beta\left(p_{j}, p_{i}\right)$ and from eq. (2.18):

$$
b r-c>0
$$

which is the usual form of Hamilton's rule.

Note that to derive the Hamilton's rule as given by eq. (2.23), we relied on four assumptions:

- individuals are perfect copies from their parents;

- $w_{i}$ incorporates additive fitness effects;

- $y_{i}$ and $h_{i}$ incorporate additive genetics; and

- there is no selection for altruism.

These assumptions are not guaranteed to hold for all models, and this is why Hamilton's rule may not prescribe the survival of altruism in some cases, which we will see in more detail in the next chapter. 


\section{Chapter 3}

\section{Two-level Fisher-Wright model: a}

\section{general framework}

The two-level Fisher-Wright model is a simple, but robust framework for explaining the emergence of pro-social traits, in a two-level population structure with selection and migration. One of the most important contributions of this framework are its generality and the capability of providing an analytical condition for the viability of the analyzed pro-social trait, under weak selection. Here, we present the general framework, which will be used in the next chapter to study the spread of altruism in a situation when there are intergroup conflicts.

\subsection{The model}

In the two-level Fisher-Wright model (TLFW), a large number of haploid individuals are divided in $g$ groups of fixed size $n$. These individuals are of two types, $A$ or $N$, in which $A$ represents the prosocial character, e.g., altruism, and $N$, the native (wild) type in the population (see fig. 3.1). There is no overlap of generations, reproduction is asexual and the type of an individual is determined from the type of his parent.

We can view this model as a three-stage model with an intragroup stage, an intergroup stage and a migration stage. In the intragroup stage, interactions inside the group produce payoffs which directly influence the fitness of individuals. In the intergroup stage, groups compete among each other, representing the natural process in which efficient groups are expected to grow faster and 


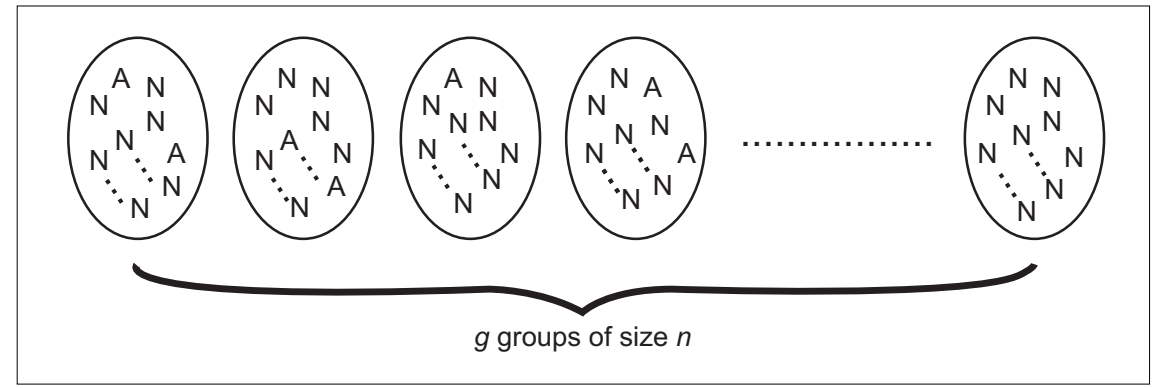

Figure 3.1: Population structure in TLFW. Individuals are divided in a large number $g$ of groups. Each group contains $n$ individuals, that are classified as $A$ or $N$, according to their actions in the interactions inside their groups.

split, while less efficient groups would go extinct. Finally, in the migration stage, individuals can migrate to other groups, possibly changing the distribution of labels $A$ and $N$ inside groups.

Before explaining how these three stages are incorporated into TLFW, we shall explain the processes underlying individual reproduction, which is tied to intragroup selection, and group reproduction, tied to intergroup selection. The individual reproduction (fig. 3.2) follows a very similar process from the original Wright-Fisher model. The offspring "choose" their parents from the parental group individuals according to the fractions of $A$ and $N$, but weighted with the ratio of fitness $\left(w_{k}^{A}\right.$ or $w_{k}^{N}$ ) over the average fitness of the group $\left(\bar{w}_{k}\right)$, in which $k$ is the number of individuals with label $A$ (this means the group is of type $k$ ). This modification allows the study of social evolution using this framework, by incorporating within-group social interactions that can lead to different fitness for $A$ and $N$ individuals.

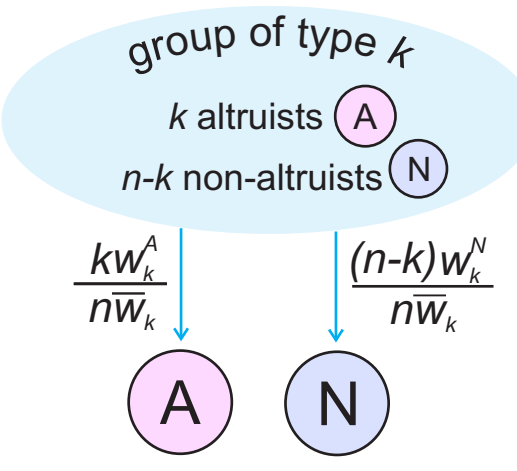

Figure 3.2: Individual reproduction in TLFW. The fraction of individuals of a label $A$ or $N$ in the next generation is proportional to the fraction of individuals $A$ or $N$ in the current generation weighted by $w_{k}^{A} / \bar{w}_{k}$ or $w_{k}^{N} / \bar{w}_{k}$

As mentioned previously, the fitness functions of $A$ and $N$ depend on the life-cycle payoffs obtained by individuals through the social interactions inside their groups. Let $v_{k}^{A}$ and $v_{k}^{N}$ be the payoffs for individuals labeled $A$ and $N$, respectively, when inside a group of type $k$. Also, we assume there is 
no positive payoff when there are only individuals of label $N$ in the group, i.e., $v_{0}^{N}=0$. Then, if $\delta$ is the parameter that controls selection strength, $\delta \in[0,1]$, the fitness functions of $A$ and $N$ are given by, respectively:

$$
w_{k}^{A}=1+\delta v_{k}^{A} \quad \text { and } \quad w_{k}^{N}=1+\delta v_{k}^{N} .
$$

The fitness functions defined above also influence the process of group reproduction (fig. 3.3), in which groups can reproduce by competing with the other groups. Analogously to the process of individual reproduction, more successful groups produce more offspring groups, and what determines the likelihood of a group of type $k$ outcompete other groups is its fitness, which is given by $\bar{w}_{k}$, the average fitness of individuals inside the group:

$$
\bar{w}_{k}=\frac{k w_{k}^{A}+(n-k) w_{k}^{N}}{n} .
$$

\section{group of type $k$ \\ $k$ altruists $A$ \\ $n-k$ altruists $N$}

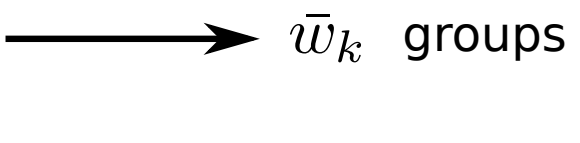

Figure 3.3: Group reproduction in TLFW. The average number of groups which has a parent group of type $k$ is given by the average fitness of individuals inside this group, $\bar{w}_{k}$.

Two matrices are necessary to model the emergence of the social trait $A: S=\left(S_{k j}\right)$, which represents the selection process and $M=\left(M_{j l}\right)$, which represents the migration process. The selection process encloses the individual reproduction and the group reproduction. Each element of matrix $S, S_{k j}$ is the expected number of groups type $j$ among the children of a group type $k$ and is given by:

$$
S_{k j}=\bar{w}_{k} \operatorname{Pr}\left(\operatorname{Bin}\left(n, \frac{k w_{k}^{A}}{n \bar{w}_{k}}\right)=j\right)
$$

in which $\bar{w}_{k}=\frac{k w_{k}^{A}+(n-k) w_{k}^{N}}{n}$ is the average fitness of a group of type $k$ and $\operatorname{Pr}(\operatorname{Bin}(n, q)=j)$ represents the probability of $j$ successful trials from $n$ trials with probability $q$ of success. Note that, in this case, $q$ represents the probability an individual of the offspring generation has social trait $A$. See fig. 3.4 for an explanation about each one of the terms of this equation.

In the migration process, individuals are chosen to migrate from its group with probability $0 \geq m \geq$ 1. Since the group size is fixed, this process is a permutation of the migrant individuals inside the 


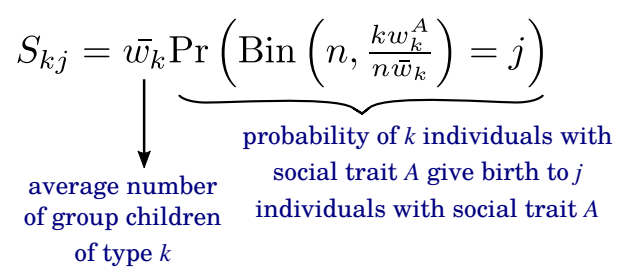

Figure 3.4: Detailed explanation about the formalization of the selection process in the terms of matrix $S$ from eq. (3.3).

groups to which they originally belonged. Note that, after migration, for each individual of social trait $A$ inside a group, there are three possibilities:

- the individual was already in the group before migration;

- the individual migrated to the group and occupied the spot left by another individual of social trait $A$;

- the individual migrated to the group and occupied the spot left by a wild type individual $N$.

Hence, each element of matrix $M, M_{j l}$, the expected number of groups of type $j$ that, due to migration, become groups of type $l$, is given by:

$$
M_{j l}=\sum_{i=0}^{n} \operatorname{Pr}(\operatorname{Bin}(j, 1-m+m p)=i) \operatorname{Pr}(\operatorname{Bin}(n-j, m p)=l-i)
$$

in which $p$ is the fraction of individuals with social trait $A$ in the population after selection (but before migration). See fig. 3.5 for an explanation about each one of the terms of this equation.



Figure 3.5: Detailed explanation about the formalization of the migration process in the terms of matrix $M$ from eq. (3.4).

The state of the TLFW model is given by the number of $A$ individuals inside a group, i.e., the type of the group, at each generation. Thus, given the initial distribution of group types, $\vec{F}(t)=$ $\left[F_{0}(t), F_{1}(t), \ldots, F_{n}(t)\right]$ at time $t=0$, and the transition matrices $S$ and $M$, it is possible to calculate 
the distribution of groups at any time $t>0$ :

$$
\vec{F}(t)=\vec{F}(t-1) S M
$$

From eq. (3.5), we can calculate the total number of individuals of social trait $A$ at any time $t>0$ :

$$
F^{A}(t)=\sum_{k=1}^{n} k F_{k}(t),
$$

in which $F_{k}(t)$ is the number of groups of type $k$ at time $t$.

Since there is no restriction about the selection strength, $\delta$, or about the distribution of individuals with social trait $A$ at $t=0$, eq. (3.5) is a general way of calculating the dynamics of the distribution of group types in a population structured in $g$ groups with fixed size $n$. Box 1 provides the steps to simulate the dynamics defined by eq. (3.5) for $T$ periods of time.

Note that to make the simulation feasible, instead of considering the absolute number of groups for each type, it is necessary to calculate the dynamics in terms of the normalized distribution of group types. In particular, $\vec{f}(t)=\left(f_{0}(t), \ldots, f_{n}(t)\right)$ is given by:

$$
\vec{f}(t)=\left(\frac{F_{0}(t)}{\sum_{k=0}^{n} F_{k}(t)}, \ldots, \ldots, \frac{F_{n}(t)}{\sum_{k=0}^{n} F_{k}(t)}\right)
$$

Also, it is important to note that the frequency $p$ of social trait $A$ in the population should be calculated after selection, so that we can use it in the calculation of the elements of matrix $M$, as in eq. (3.4). 
Box 1: Step-by-step numerical simulation of the general TLFW.

1. Initialize the fractions of group types in the population $\vec{f}(0)$.

For $t$ in $1,2 \ldots T$, run steps from 2 to 4 :

2. Calculate matrix $S$.

3. Multiply the group distribution vector $\vec{f}(t-1)$ by the selection matrix $S$, obtaining the distribution of groups after selection but before migration given by $\vec{f}^{S}(t-1)=\vec{f}(t-1) S$.

4. Calculate the fraction of individuals of social trait $A$ in the population, given by $p=$ $\sum_{k=0}^{n} k f_{k}^{S}(t-1)$, in which $f_{k}^{S}(t-1)$ is the frequency of group type $k$ after selection.

5. Calculate matrix $M$ using $p$ defined in the step 4.

6. Multiply the group distribution vector $f_{k}^{S}(t-1)$ by the migration matrix $M$, obtaining the distribution of groups after migration given by $\vec{f}(t)=f_{k}^{S}(t-1) M$.

\subsubsection{Evolutionary stages}

The above method for calculating the evolution of social trait $A$ in a structured population is general, since it does not rely on assumptions about the selection strength or the initial number of individuals of social trait $A$. However, it can be useful to divide the evolution of social trait $A$ into different stages regarding the number of individuals of trait $A$ in the population, namely, Very Early Stage (VES), Early Stage (ES), and Late Stage (LS), which are represented in a timeline shown in fig. 3.6.

At Very Early Stage, the mutation from $N$ to $A$ just occurred and there is no way of predicting the fate of that rare mutation, since there are large fluctuations of the fraction $p$ of individuals of trait $A$, which can lead to extinction even when the viability condition is satisfied. At Early Stage, $p$ is still very small and the viability condition determines whether the process will be extinct or not. If individuals of trait $A$ survive, the distribution of group types reaches a demographic equilibrium at the so-called Stationary Early Stage, after which the fractions of group types increase. Finally, at Late Stage, the absolute number of individuals of trait $A$ in the population is of the same order as the number of groups $g$.

Here we focus on approximations for Early Stage, making use of the distribution of group types at Stationary Early Stage. An analysis of the viability conditions under weak selection at Late Stage can be found in [84]. 


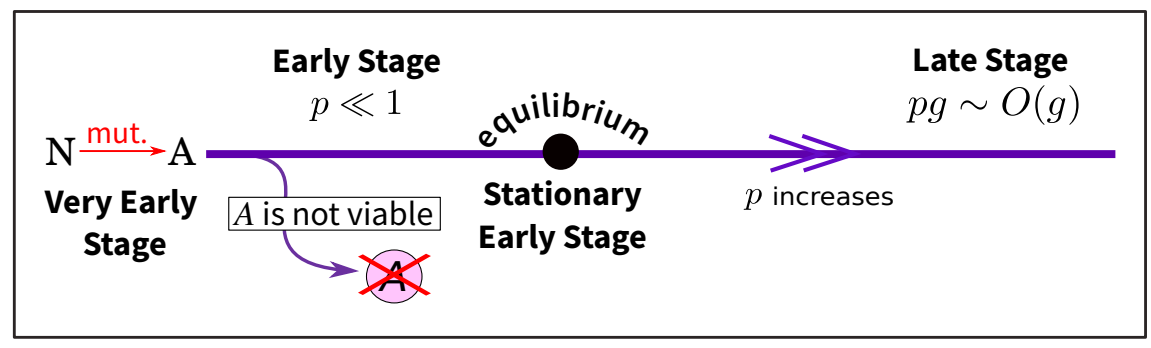

Figure 3.6: An evolutionary timeline representing the stages to which social trait $A$ is subject, when it starts from a single mutation at VES inside a monomorphic population dominated by trait $N$. In ES, the fate of the mutation $A$ is decided and $A$ can be extinct or survive with its distribution reaching a demographic equilibrium at SES. LS occurs when the number of individuals of trait $A$ cannot be neglected compared to the total number of groups $g$.

\subsection{Early Stage approximations}

When we are concerned only with the emergence of the social trait $A$, we can assume individuals $A$ are very rare in the population. This assumption is used in the migration stage, in which we assume the probability that a group of type $k>0$ (i.e. with one or more individuals of type $A$ ) receives another individual of trait $A$ through migration is so small that it can be neglected. Hence, it is possible to build a simplified version of this process (see fig. 3.7).

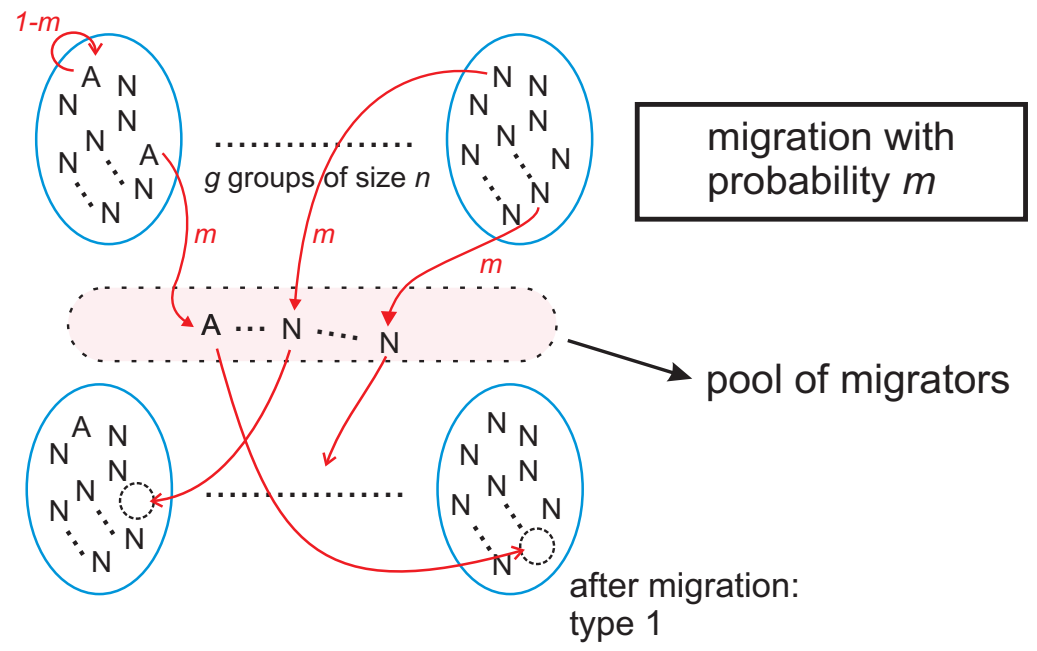

Figure 3.7: Simplified migration process when individuals $A$ are very rare in the population. We assume that, in the migration process, it is very unlikely that a group of type $k>0$ receives a migrant individual of trait $A$.

With this simplification, matrix $M$ can be decomposed as the sum of two other matrices, $M^{A}$ and $M^{B}$, as shown in eq. (3.8). Matrix $M^{A}$ represents the process of individual migrations with rate $m$, which implies that altruists stay in their original group with probability $1-m$ (eq. (3.9)). On the other hand, matrix $M^{B}$ describes the assignment of migrant altruists to new groups, with the restriction that, due to the small frequency of altruists in the population, migrants would only go 
to groups in which there were no individuals of social trait $A$ (eq. (3.10)).

$$
\begin{gathered}
M=M^{A}+M^{B} \\
M_{j l}^{A}=\operatorname{Pr}(\operatorname{Bin}(j, 1-m)=l) \\
M_{j l}^{B}= \begin{cases}=m j, & \text { if } l=1 \\
=0, & \text { if } l \neq 1\end{cases}
\end{gathered}
$$

Let $R=S M$, then, the element $R_{k l}$ corresponding to the $k$-th row and the $l$-th column of matrix $R$ is the average number of groups with type $k$ that become groups of type $l$ due to combined effects of selection and migration. $R_{k l}$ can be calculated from eqs. (3.3) and (3.8) and it is given by:

$$
\begin{aligned}
R_{k l} & =\sum_{j=0}^{n} S_{k j} M_{j l} \\
& = \begin{cases}\sum_{j=0}^{n} \bar{w}_{k} \operatorname{Pr}\left(\operatorname{Bin}\left(n, \frac{k w_{k}^{A}}{n \bar{w}_{k}}\right)=j\right)[\operatorname{Pr}(\operatorname{Bin}(j, 1-m)=1)+m j], & \text { if } l=1 \\
\sum_{j=0}^{n} \bar{w}_{k} \operatorname{Pr}\left(\operatorname{Bin}\left(n, \frac{k w_{k}^{A}}{n \bar{w}_{k}}\right)=j\right) \operatorname{Pr}(\operatorname{Bin}(j, 1-m)=l), & \text { if } l \neq 1 .\end{cases}
\end{aligned}
$$

Since all summation terms are non-negative, $R_{k l} \geq 0, \forall(k, l) \in[0, n] \times[0, n]$, with $[0, n] \subset \mathbb{N}$. This means $R_{k l}=0$ can only be zero if all summation terms are zero, which happens only when $k=0$ and $l>0$. Thus, $k=0$ is an absorbing state: when there are no individuals with social trait $A$ in the group, there are no offspring with trait $A$ and the possibility an individual with trait $A$ comes to the group due to migration is negligible, because we are assuming individuals with trait $N$ are predominant in the population.

From eq. (3.5), we can see the relation between the current distribution of group types and matrix $R=S M$. Let $\vec{f}_{*}(t)=\left(f_{1}(t), \ldots, f_{n}(t)\right)$ be the normalized distribution of group types at time $t$. Note that $\vec{f}_{*}(t)$ is different from $\vec{f}(t)$ by one dimension: it does not have the first dimension of $\vec{f}(t)$, given by $f_{0}(t)$. Despite this difference, by calculating $\vec{f}_{*}(t)$ we can fully determine the distribution of group types, since $\sum_{k=0}^{n} f_{k}(t)=1$ and, thus, $f_{0}(t)=1-\sum_{k=1}^{n} f_{k}(t)$. Then, we can write the dynamics for $\vec{f}_{*}(t)$ as:

$$
\vec{f}_{*}(t)=\vec{f}_{*}(t-1) R_{*},
$$

in which $R_{*}$ is a reduced matrix with size $n \times n$, and is obtained by removing the first line, $\left(R_{0 j}\right)_{j \in\{0,1, \ldots, n\}}$, and the first column, $\left(R_{i 0}\right)_{i \in\{0,1, \ldots, n\}}$ of matrix $R=S M$. 
Because $R_{*}$ does not contain the first row and first column of matrix $R$, all of its elements are strictly positive and, from Perron-Frobenius Theorem [64], it follows that:

- $R_{*}$ has a dominant eigenvalue $\rho$, which is real, positive and larger in absolute value than all other eigenvalues;

- there are two eigenvectors associated with the eigenvalue $\rho$ : a single left eigenvector $\nu$ and a single right eigenvector $\zeta$, with all of their entries strictly positive;

- $\lim _{k \rightarrow \infty} \frac{R_{*}^{k}}{\rho^{k}}=\zeta \nu^{\prime}$, in which $\zeta$ and $\nu$ are normalized so that $\nu^{\prime} \zeta=1$, and $\nu^{\prime}$ is the transpose of $\nu$.

One important feature of this model is that it provides a relatively simple viability condition (Theorem 3.1) for the emergence of the cooperative trait.

Theorem 3.1. Let $R_{*}$, the reduced matrix of matrix $R=S M$, with size $n \times n$, obtained by removing the first line, $\left(R_{0 j}\right)_{j \in\{0,1, \ldots, n\}}$, and the first column, $\left(R_{i 0}\right)_{i \in\{0,1, \ldots, n\}}$ of matrix $R$. The condition for the viability of the social trait $A$ is given by:

$$
\rho(m)>1
$$

in which $\rho$ is the dominant eigenvalue of matrix $R_{*}$ and $m$ is the migration rate.

Furthermore, for $t \gg 1, f(t)=C \rho^{t} \nu$, in which $C$ is a constant and $\nu$ is the left eigenvector associated with the dominant eigenvalue $\rho$ of matrix $R_{*}$.

The proof of this theorem can be found in be found in [101] and follows from the application of Theorem 7.1, Chapter II, of Harris [45], on the condition for the survival of a multitype branching process (MBP). Viewed as a MBP, $R_{*}$ is the first moment of the MBP, whose types are the types of groups (from 1 to $n$ ). Harris theorem states that if the dominant eigenvalue of matrix $R_{*}$ is larger than one, then, there is positive probability that the types will survive. The existence of the dominant eigenvalue is guaranteed by the Perron-Frobenius Theorem [64], for nonnegative matrices.

\section{Another interpretation of the viability condition}

We can also write the viability condition $\rho>1$ in terms of the fraction of individuals with social trait $A$ in the population, given by $p(t)=\sum_{k=0}^{n} k f_{k}(t)$. From the replicator dynamics for viability 
selection, we can write the condition of viability as:

$$
\bar{W} \Delta p=p\left(W^{A}-\bar{W}\right)>0
$$

in which $\bar{W}=p W^{A}+(1-p) W^{N}$ is the average fitness over all group types and $W^{A}\left(W^{N}\right)$ is the average fitness of an individual of social trait $A(\operatorname{trait} N)$.

Since we are assuming $p \ll 1, \bar{W}=1$, i.e., on average, we expect to find groups composed only by individuals of wild type $N$. On the other hand, the probability of a random individual of trait $A$ is in a group of type $k$ is given by $k f_{k} /\left(\sum_{k=1}^{n} k f_{k}\right)$, so that the average fitness of individuals of trait $A$ is given by $W^{A}=1+\frac{\delta \sum_{k=1}^{n} k f_{k} v_{k}^{A}}{\sum_{k=1}^{n} k f_{k}}$. Replacing these values in both sides of the equality in eq. (3.14) produces an approximated expression for $\Delta p$ :

$$
\Delta p=\delta p \frac{\sum_{k=1}^{n} k \nu_{k} v_{k}^{A}}{\sum_{k=1}^{n} k \nu_{k}}
$$

in which we wrote $f_{k}$ as $\nu_{k}$, since $f(t)$ is driven towards multiples of $\nu$. Hence, the viability condition is given by $\Delta p>0$ is equivalent to $\rho>1$, when we assume a stationary regime, with $t \gg 1$ and $p \ll 1$.

\subsubsection{Limit of weak selection}

If $\delta=0$ and individuals of social trait $A$ are rare in the population, $v_{k}^{A}=v_{k}^{N}$ and, thus, $p(t)=$ $\sum_{k=0}^{n} k f_{k}(t)$ is constant. Since, from theorem 3.1, for large $t, f(t)=C \rho^{t} \nu$, this means that $\rho^{0}=1$. Also, , when $\delta=0$, we denote matrix $R_{*}$ and the left eigenvector $\nu$ as, respectively, $R_{*}^{0}$ and $\nu^{0}$.

We can think of weak selection, $\delta \rightarrow 0$, as a a perturbation of the case $\delta=0$. Because of the continuity of eigenvalues and eigenvectors, if $\delta \rightarrow 0$, then, $\rho \rightarrow 1$ and $\nu \rightarrow \nu^{0}$. Since $\nu^{0}$ does not depend on the payoff $v_{k}^{A}$ and $v_{k}^{N}$, we can expect the influence of $v_{k}^{A}$ and $v_{k}^{N}$ in $\nu$ vanishes as $\delta$ approaches zero.

Due to this separation of time scales, assuming weak selection, it is possible to write the viability condition $\Delta p>0$ (according to eq. (3.15)) as:

$$
\sum_{k=1}^{n} \pi_{k} v_{k}^{A}>0
$$


in which $\pi_{k}$ can be interpreted as the probability of an individual of social trait $A$ belongs to a group of type $k$ and is given by $\pi_{k}=\frac{k \nu_{k}^{0}}{\sum_{j=1}^{n} j \nu_{j}^{0}}$.

In Early Stage, when $A$ individuals are very rare in the population, we can assume individuals of trait $N$ are very likely to be in groups with only individuals of the same trait $N$. This also means it is almost certain that if you consider a focal individual of trait $A$, all individuals of trait $A$ inside his group are related to him, i.e., are IBD (identical by descent). Hence, letting $K$ be the random variable of the number of IBD individuals inside a group, we can write distribution $\pi$ in the following way:

$$
\pi_{k}=\operatorname{Pr}(K=k \mid A)
$$

in which $A$ indicates we are considering a focal individual of trait $A$ and $k \in\{1,2, \ldots, n\}$.

Note that this equation is only valid in the stationary early stage, when the distribution of group types is already at demographic equilibrium $\nu^{0}$. We should, then, calculate the demographic equilibrium of distribution $\pi$, for $\delta=0$.

First, let us define how we calculate the number of individuals inside a group who are IBD to a certain focal individual, member of that group. Note that an individual is IBD to another if they share the same parent or if their parents are IBD, and provided that none of them are migrants. Thus, we can express the current number of individuals of trait $A$ who are IBD in the group of the focal individual of trait $A$ in the current generation $u, K_{u}$ in terms of the number of individuals of trait $A$ in the previous generation $u-1, K_{u-1}$ (see fig. 3.8).

Note that the assumption $\delta=0$ is used to calculate the probability of an individual being of social trait $A$. If $\delta=0$, evolution occurs as a consequence of random genetic drift and can be modeled as a Wright-Fisher process. In the Wright-Fisher model, reproduction is a gene-pool process, i.e., the offspring are metaforically put into a pool, and choose a parent $A$ according to its fraction in the population (which is the equivalent to the group the focal individual in TLFW). This is the same as a sampling with replacement, therefore, the number of $A$ s after reproduction has a Binomial distribution with size $n$ and probability $p=K_{u-1} / n$, in which $K_{u-1}$ is the number of $A$ s before reproduction (in the previous generation $u-1$ ).

Hence, the sequence of random variables $\left(K_{0}, K_{1}, K_{2}, \ldots K_{t}\right)$ is a Markov process and it can be described with a transition matrix $Q$, in which each element $Q_{i j}$ is the probability of the transition from $K_{u-1}=i$ to $K_{u}=j$. Following the two possibilities for the focal individual of trait $A$ (for 


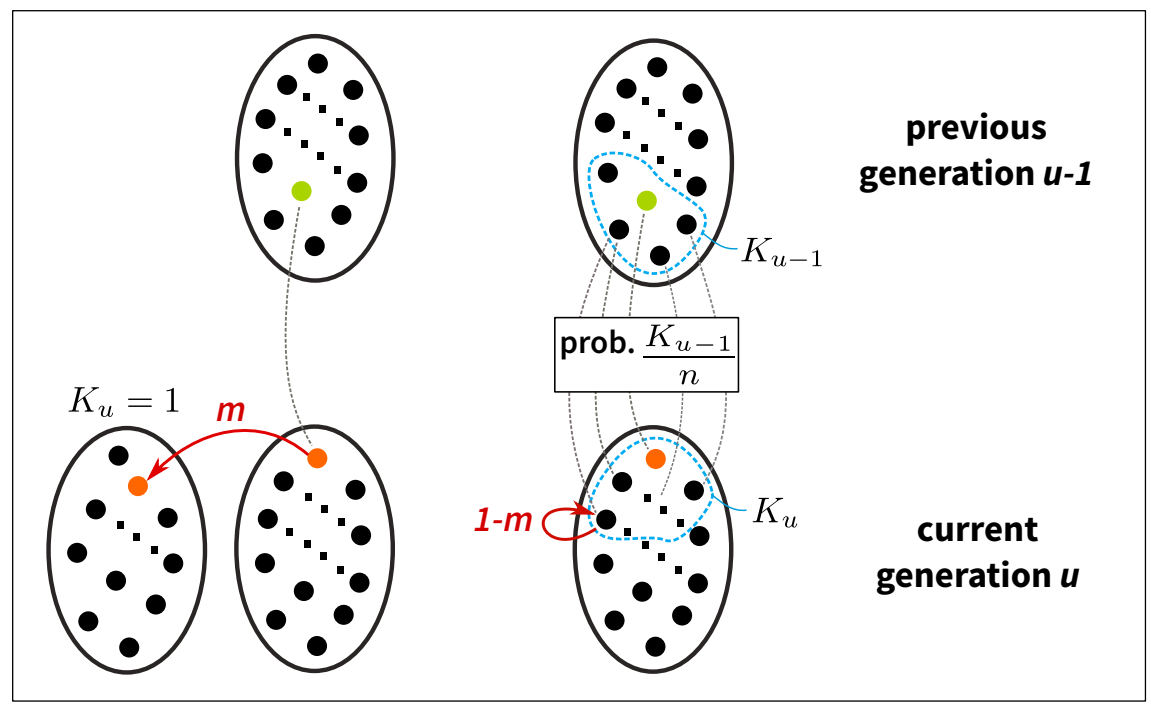

Figure 3.8: Explanation about the calculation of $K_{u}$, the number of individuals who are IBD in a group to which the chosen focal individual of trait $A$ belongs, at current generation $u$, from the number of individuals of trait $A$ in the previous generation $u-1, K_{u-1}$. The focal individual is represented by the orange dot, while her ancestral in the previous generation is the green dot. In the left, we depict the situation when the focal individual migrates to another group: since $p \ll 1$, she arrives to a group in which she is the only individual with trait $A$, so that $K_{u}=1$. In the right, the assumption of $\delta=0$ determines the probability $K_{u-1} / n$ with which an individual is of trait $A$ when he descends from a group with $K_{u-1}$ individuals of trait $A$. In this case, the current generation will have $K_{u}$ individuals who are IBD if their parents are among the $K_{u-1}$ individuals of the previous generation and are not subject to migration.

simplicity, below, we refer to him as "focal individual") shown in fig. 3.8, we can separate the calculation of $Q_{i j}$ into two cases:

1. if $j=1$, there are two possibilities:

- the focal individual is a migrant, which occurs with probability $m$; or

- the focal individual does not migrate, which occurs with probability $1-m$, and individuals of trait $A$ from the previous generation did not leave other offspring (except for the focal individual) who remained in the group, which occurs with probability $\operatorname{Pr}(\operatorname{Bin}(n-1,(1-m) i / n)=0)$;

2. if $j=2, \ldots, n$ : this can only happen if the focal individual remains in his group (occurs with probability $1-m$ ) and exactly $j-1$ of the other individuals inside the group remained in the group and have parents who are among the $i$ individuals of trait $A$ in the previous generation, which occurs with probability $\operatorname{Pr}(\operatorname{Bin}(n-1,(1-m) i / n)=j-1)$. 
These two cases are reflected in the formula for $Q_{i j}$ :

$$
Q_{i j}= \begin{cases}m+(1-m) \operatorname{Pr}(\operatorname{Bin}(n-1,(1-m) i / n)=0), & \text { if } j=1 \\ (1-m) \operatorname{Pr}(\operatorname{Bin}(n-1,(1-m) i / n)=j-1), & \text { if } j \neq 1,\end{cases}
$$

in which $i \in\{1,2, \ldots n\}$ and $j \in\{1,2, \ldots n\}$.

Given $Q$, then, it is possible to calculate the demographic equilibrium $\pi=\pi^{*}$, with $\pi^{*}=\left(\pi_{1}^{*}, \pi_{2}^{*}, \ldots, \pi_{n}^{*}\right)$, at SES (Stationary Early Stage) through the solution of:

$$
\pi=Q \pi
$$

with $\sum_{k=1}^{n} \pi_{k}=1$.

\section{Viability condition based on relatedness}

From eq. (3.17), we can see $\pi_{k}$ is the probability of the number of individuals who are IBD to the focal individual of social trait $A$ inside a group being $k$. However, from the explanation about how to calculate $K$ in fig. 3.8, it is possible to see that in Early Stage, the number of individuals who are IBD to the focal individual of social trait $A$ inside a group is exactly the number of individuals of social trait $A$ inside that group. This means that, by abusing the notation and representing both quantities with the same random variable $K$, we can write $\pi_{k}$ as the probability of $K$ being equal to $k$ without conditioning on the focal individual at eq. (3.17).

Previously we mentioned the notion of IBD (identical by descent), which can be defined as the genetic identity two individuals from the same generation $t$ share due to a coalescent event at some past generation $t-i$, with $i>0$, before a migration event in one of the two lineages. This means those two individuals share a common ancestral through which they became part of their group. Thus, in ES (when $p \ll 1$, i.e., $A$ is a rare phenotype in the population), if $D$ is a binary random variable which determines whether, given a focal individual of trait $A$, a co-focal individual is IBD to him $(D=1)$ or not $(D=0)$, then, relatedness can be defined as:

$$
r_{t}=\mathrm{P}_{t}(D=1 \mid A),
$$

in which the subscript $t$ makes the time dependence explicit. 
By assuming weak selection, $\delta \rightarrow 0$, however, we can assume there is a separation of time scales, so that a demographic equilibrium is achieved through the process of genetic drift (when $\delta=0$ and $\left.v_{k}^{A}=v_{k}^{N}\right)$ and, then, selection starts to affect this distribution at a rate of order $\delta$. This implies that $r_{t}=r$, i.e., relatedness is independent of time.

In TLFW, we can then calculate $r$, by calculating the probability $P(D=1 \mid A)$. As explained before, under weak selection, $w_{k}^{A} \approx w_{k}^{N}$, which means the probability of the co-focal individual having the same parent of the focal individual is $1 / n$ (is independent of the fact that the focal individual is of social trait $A$ ). Conversely, the probability that they have different parents is $1-1 / n$ and, in this case, they will only be IBD if those two parents are IBD to one another, which occurs with probability $r$. Additionally, in both cases, the two individuals are only IBD to each other if they are not migrants, which occurs with probability $(1-m)^{2}$. Thus:

$$
r=(1-m)^{2}\left[\frac{1}{n}+\left(1-\frac{1}{n}\right) r\right] \quad \Longrightarrow \quad r=\frac{(1-m)^{2}}{n-(1-m)^{2}(n-1)}
$$

The association of relatedness $(r)$ to the migration rate $(m)$ makes it possible to write the viability condition obtained for ES, $\rho>1$ in terms of $r$, when selection is weak:

$$
r>r_{c} \Longleftrightarrow r>\frac{\left(1-m_{c}\right)^{2}}{n-\left(1-m_{c}\right)^{2}(n-1)}
$$

in which $m_{c}$ is the critical migration rate for which $\rho=1$.

\subsubsection{Limit of large $n$ and small $m$ under weak selection}

Under weak selection and in the limit of $n \rightarrow \infty$ and $m \rightarrow 0$, we can calculate the distribution $\pi$ at equilibrium, showing that it is distributed as a Beta distribution with parameters $\alpha=1$ and $\beta=2 n m$. Here we indicate how to prove this result by using the moments of $\pi$.

First, we would like to generate a recurrent expression for the $l$-th moment of the random variable $K$ at equilibrium, when $\pi=\pi^{*}$. This can be done by following the general definition of the $l$-th moment of a random variable $K$, given by: $E\left(K^{l}\right)=\sum_{k=1}^{n} k^{l} \pi_{k}$, in which $\pi_{k}=\mathrm{P}(K=k)$.

Because of the nature of the elements of matrix $Q$ (see eq. (3.18)), instead of calculating the $l$-th moment for $K_{u}$, the number of individuals who are IBD to a focal individual of trait $A$ in generation $u$, it is more convenient to first write an expression for $K_{u}-1$. 
In this way, since the first value $K_{u}-1$ can take is zero, the first term of the expression can be eliminated. Then:

$$
\begin{aligned}
\mathrm{E}\left[\left(K_{u}-1\right)^{l}\right] & =\sum_{j=1}^{l}(1-m) j^{l} \mathrm{P}\left[\operatorname{Bin}\left(n-1,(1-m) K_{u-1} / n\right)=j\right] \\
& =(1-m) \mu_{l}^{B}
\end{aligned}
$$

in which $\mu_{l}^{B}$ is the $l$-th moment of a random variable distributed as $\operatorname{Bin}(N, p)$, given by eq. (3.25) from box 2 , with parameters $N=n-1$ and $p=(1-m) \frac{K_{u-1}}{n}$.

Box 2: Moments for the Binomial distribution

For a random variable distributed as $\operatorname{Bin}(N, p)$, the moment-generating function is given by the following expression:

$$
\mathcal{M}(t)=\sum_{k=0}^{N} e^{t}\left(\begin{array}{l}
N \\
k
\end{array}\right) p^{k}(1-p)^{N-k}=\left(p e^{t}+(1-p)\right)^{n} .
$$

From the moment-generating function, it is possible to calculate any moment of order $l$ by calculating the $l$-th derivative of $\mathcal{M}(t)$ at $t=0$, i.e., $\mu_{l}=\left.\frac{d^{l} \mathcal{M}(t)}{d t^{l}}\right|_{t=0}$.

By calculating each one of the moments, it is possible to derive a general explicit formula for the l-th moment of the Binomial distribution, $\mu_{l}^{B}$ :

$$
\mu_{l}^{B}=\sum_{k=0}^{N} N_{k} S(l, k) p^{k}
$$

in which $N_{k}=\frac{N !}{k !}$ and $S(l, k)$ is the Stirling number of the second kind, which counts the number of ways one can partition $l$ elements into $k$ nonempty sets, and it is calculated from $S(l, k)=\frac{1}{k !} \sum_{j=0}^{k}\left(\begin{array}{c}k \\ j\end{array}\right)(k-j)^{l}$.

By replacing $s=1-m$ and eq. (3.25) in eq. (3.23), we obtain a formula for the $l$-th moment of $K_{u}-1:$

$$
\mathrm{E}\left[\left(K_{u}-1\right)^{l}\right]=s \sum_{j=1}^{l} S(l, j)(n-1)_{j} s^{j+1} \frac{\mathrm{E}\left(K_{u-1}^{j}\right)}{n} .
$$

Note that a binomial expansion of $\left(K_{u}-1\right)^{l}$ yields $\sum_{j=0}^{l}\left(\begin{array}{l}l \\ j\end{array}\right)(-1)^{l-j} K_{u}^{l}$, which means the left hand side of eq. (3.26) can also be written as:

$$
\mathrm{E}\left[\left(K_{u}-1\right)^{l}\right]=\sum_{j=0}^{l}\left(\begin{array}{l}
l \\
j
\end{array}\right)(-1)^{l-j} \mathrm{E}\left(K_{u}^{j}\right) .
$$


Since we are interested in calculating this quantity at the demographic equilibrium, $K_{u}$ and $K_{u-1}$ have the same distribution $\pi^{*}$ and, thus, $\mathrm{E}\left[\left(K_{u}-1\right)^{l}\right]=\mathrm{E}\left(K_{u-1}^{l}\right)=\mu_{l}$. By rewriting eq. (3.26) and eq. (3.27) using $\mu_{l}$ and combining them, we can get an expression for $\mu_{l}$ in function of $\mu_{j}$, $j=1,2, \ldots, l$ :

$$
\begin{gathered}
\sum_{j=0}^{l}\left(\begin{array}{l}
l \\
j
\end{array}\right)(-1)^{l-j} \mu_{j}=s \sum_{j=1}^{l} S(l, j)(n-1)_{j} s^{j+1} \frac{\mu_{j}}{n} \\
\mu_{l}+(-1)^{l}+\sum_{j=1}^{l-1}\left(\begin{array}{l}
l \\
j
\end{array}\right)(-1)^{l-j} \mu_{j}=\frac{(n-1)_{l}}{n^{l}} s^{l+1} \mu_{l}+s \sum_{j=1}^{l-1} S(l, j)(n-1)_{j} s^{j+1} \frac{\mu_{j}}{n},
\end{gathered}
$$

in which we used $S(l, l)=1$ and $\mu_{0}=1$.

Solving eq. (3.28) for $\mu_{l}$ yields:

$$
\mu_{l}=\frac{(-1)^{l+1}+\sum_{j=1}^{l-1}\left[\left(\begin{array}{l}
l \\
j
\end{array}\right)(-1)^{l-j+1} \mu_{j}+S(l, j)(n-1)_{j} s^{j+1} \frac{\mu_{j}}{n}\right]}{1-\frac{(n-1)_{j} s^{l+1}}{n^{l}}} .
$$

With eq. (3.29), we can calculate moments of any order by calculating each one of the lower order moments. For instance, the calculation of the first moment $(l=1)$ is straightforward and gives us:

$$
\mu_{1}=\frac{n}{n-(n-1)(1-m)^{2}}
$$

Theorem 3.2 enunciates formally the result previously mentioned about the distribution $\pi^{*}$.

Theorem 3.2. Let $X$ be the frequency of altruists of a group, given by $K / n$. If $\delta \rightarrow 0, m \rightarrow 0$ and $n \rightarrow \infty$, then, the distribution of $X$ follows a Beta distribution, with parameters $\alpha=1$ and $\beta=2 n m, \operatorname{Beta}(1,2 n m):$

$$
P(X=x)=2 n m(1-x)^{2 n m-1}
$$

This result can be proved by showing that the moments of $K / n$ converge to the moments of a Beta distribution with parameters $\alpha=1$ and $\beta=2 m n$, when $n \rightarrow \infty$ and $m \rightarrow 0$ :

$$
\lim _{\substack{n \rightarrow \infty \\ m \rightarrow 0}} \frac{\mu_{l}}{n^{l}}=\frac{l !}{\prod_{j=1}^{l}(2 m n+j)}
$$


Equation (3.32) can be proven by induction on $l$ using the formula for the $l$-th moment, given by eq. (3.29). A full proof can be found in [85, 101].

\subsection{Examples of applications}

In this section, we consider the case in which the social trait $A$ represents altruism. In order to model the evolution of altruism under the TLFW framework, we will assume individuals interact inside the group as in a public goods game (see appendix section A.1.1 for more details about it). Here we focus on three different public goods games: OOPG (Others-Only Public Goods Game), IPG (Iterated Public Goods Game) and THR (Threshold Public Goods Game).

Before showing the results of the application of the TLFW into the different payoff functions, we write the equations for the payoffs of $A, v_{k}^{A}$, and $N, v_{k}^{N}$, for every one of the games OOPG, IPG and THR. For OOPG, payoffs for $A$ and $N$ inside a group with size $n$ with $k$ altruists are given by:

$$
\begin{aligned}
& v_{k}^{A}=b(k-1) /(n-1) \\
& v_{k}^{N}=b k /(n-1),
\end{aligned}
$$

in which $b>0$ and $c>0$.

For IPG, payoffs for $A$ and $N$ inside a group with size $n$ with $k$ altruists are given by:

$$
\begin{gathered}
v_{k}^{A}=\left\{\begin{array}{cc}
-c+\frac{b(k-1)}{n-1}, & \text { if } k \leq \theta, \\
T\left(-c+\frac{b(k-1)}{n-1}\right), & \text { if } k>\theta,
\end{array}\right. \\
v_{k}^{N}= \begin{cases}\frac{b k}{n-1}, & \text { if } k \leq \theta, \\
T \frac{b k}{n-1}, & \text { if } k>\theta,\end{cases}
\end{gathered}
$$

in which $b>0, c>0, \theta>0$ and $T>0$.

For THR, payoffs for $A$ and $N$ inside a group with size $n$ with $k$ altruists are given by:

$$
v_{k}^{A}= \begin{cases}-c, & \text { if } k<\theta, \\ -c+b, & \text { if } k \geq \theta,\end{cases}
$$




$$
v_{k}^{N}= \begin{cases}0, & \text { if } k<\theta, \\ b^{\prime}, & \text { if } k \geq \theta .\end{cases}
$$

in which $b>0, b^{\prime}>0, c>0$ and $\theta>0$.

In fig. 3.9, we show how these different payoffs shown above vary according to the number of altruists in the group. We can see that it is always disadvantageous to be an altruist compared to being a non-altruist. While the fitness functions for $A$ and $N$ are linear on $k$ for OOPG, for IPG and THR it is easy to identify the threshold at which the collective good starts to be produced by the non-linear increase on the fitness functions evaluated around that threshold.
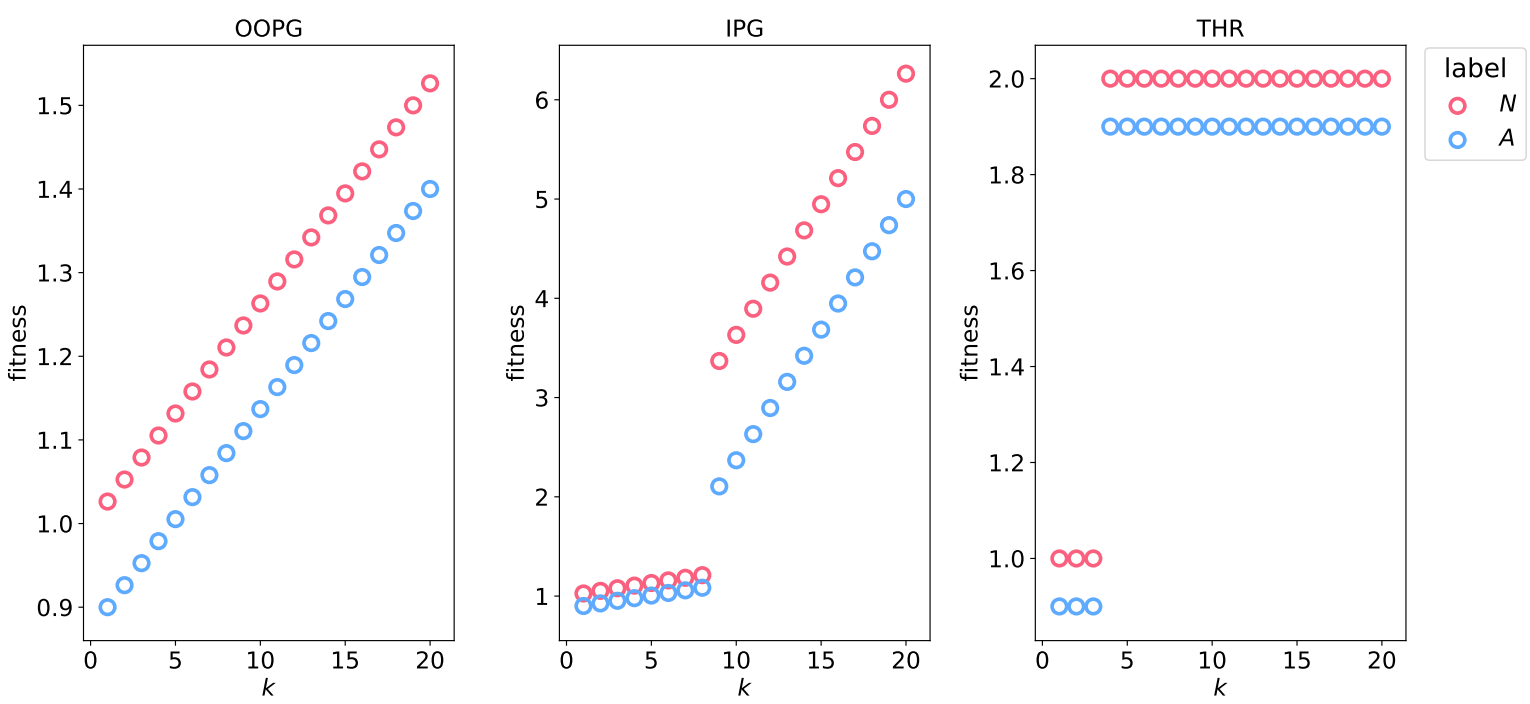

Figure 3.9: Fitness functions for an altruist individual $(A)$ and a non-altruist individual $(N)$ versus number of altruists $(k)$ for different public goods games. At each panel, a different public goods game is considered: from left to right, respectively, OOPG (Others-Only Public Goods Game), IPG (Iterated Public Goods Game) and THR (Threshold Public Goods Game). For all public goods games, we considered $n=20$ and $\delta=0.1$. OOPG parameters: $b=5, c=1$; IPG parameters: $b=5, c=1, T=10, \theta=8$; THR parameters: $b=10, b^{\prime}=10, c=1, \theta=4$.

In the TLFW framework, one of the stages involves group reproduction and depends on the average fitness $\left(\bar{w}_{k}\right)$. In fig. 3.10, it is possible to see the behavior of the average fitness as the number of altruists in the group increases. For OOPG, it is very clear that larger $\delta$ makes the average fitness level increase. For IPG and THR, if the number of altruists does not reach the threshold, different levels of $\delta$ have very limited influence on $\bar{w}_{k}$. If the number of altruists is larger than the threshold, however, the behavior is similar to OOPG, and, the larger $\delta$, the points are higher. While the average fitness for OOPG and IPG are increasing functions of $k$, for THR, the average fitness decreases as $k$ increases, except for $k$ around the threshold $\theta$, as when $\theta$ is reached, the fitness functions are much larger, as seen in fig. 3.9. 

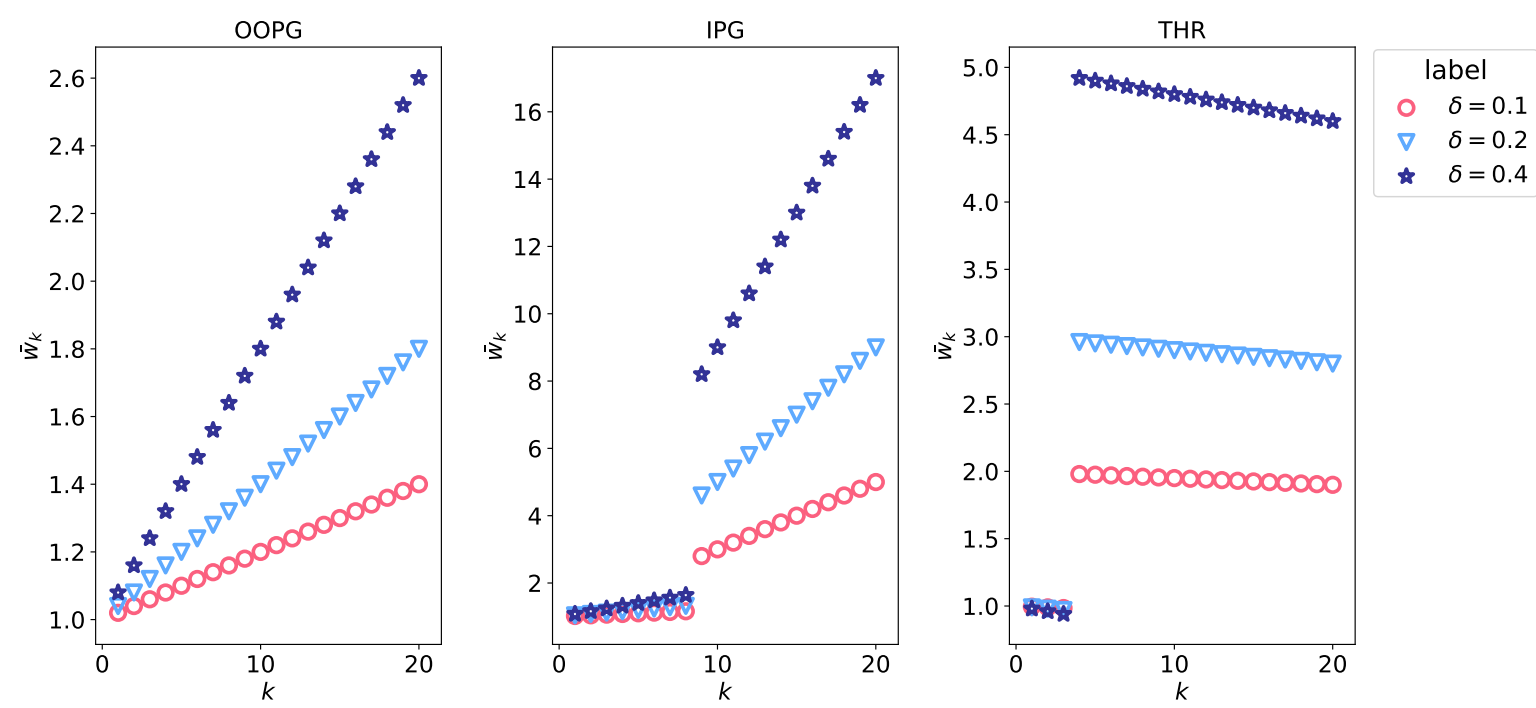

Figure 3.10: Average fitness $\left(\bar{w}_{k}\right)$ versus number of altruists $(k)$ at three different selection strength $(\delta)$ levels for different public goods games. At each panel, a different public goods game is considered: from left to right, respectively, OOPG (Others-Only Public Goods Game), IPG (Iterated Public Goods Game) and THR (Threshold Public Goods Game). For all public goods games, we considered $n=20$. OOPG parameters: $b=5, c=1$; IPG parameters: $b=5, c=1, T=10, \theta=8$; THR parameters: $b=10, b^{\prime}=10, c=1, \theta=4$.

In fig. 3.11, following the discussion in section 3.2.1, we show how the behavior of group type distribution differ between two different selection strength $(\delta)$ levels. While, when $\delta$ is small, different payoff functions yield very similar group type distributions, when $\delta$ is sufficiently large, their distributions grow apart from each other.

We can also use the viability condition given by theorem 3.1, $\rho>1$, to find which sets of parameters allow altruism to emerge. In fig. 3.12, we see how the dominant eigenvalue of matrix $R$ (as given by eq. (3.11)), $\rho$ behave for different values of migration rates $(m)$ and for three different selection strength levels $(\delta)$. In this figure, the parameters related to the benefit, cost, threshold (for IPG and THR) and number of iterations (for IPG) are the same as the ones in fig. 3.9. As the fitness functions for $A$ and $N$ are lower and closer to each other when there are few altruists in the group, when the game is IPG or THR, we can see that a wider range of migration rates meet the viability criteria, compared to OOPG. We can also see the larger $\delta$, the higher is the maximum migration rate $m$ such $\rho>1$, which is intuitive from fig. 3.10 . 

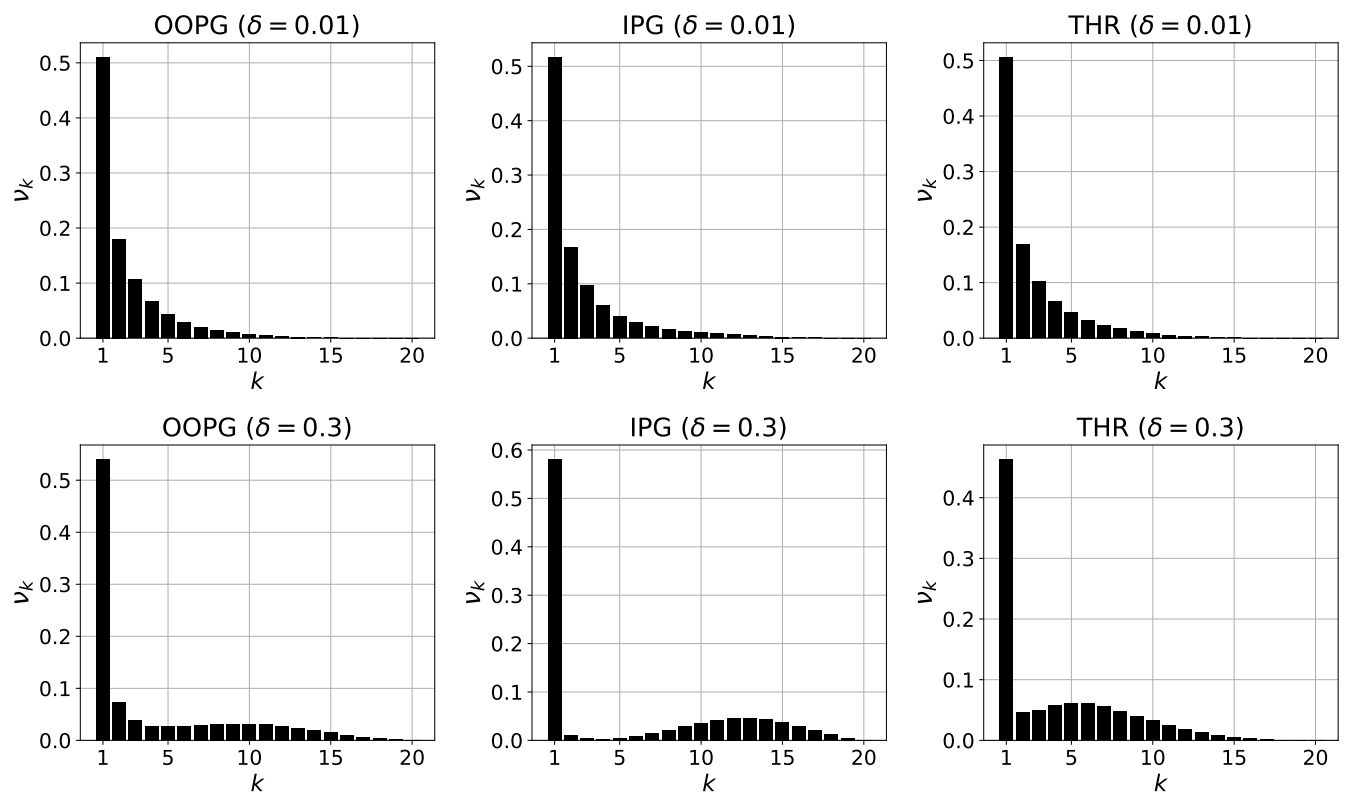

Figure 3.11: Distribution of group types $\left(\nu_{k}\right)$ for different public goods games and different values of selection strength $(\delta)$. OOPG represents the others-only public goods game; IPG is the iterative public goods game; and THR is the threshold public goods game. For all public goods games, we considered $n=20$ and $m=0.1$. OOPG parameters: $b=5, c=1$; IPG parameters: $b=5, c=1$, $T=10, \theta=8$; THR parameters: $b=10, b^{\prime}=10, c=1, \theta=4$.
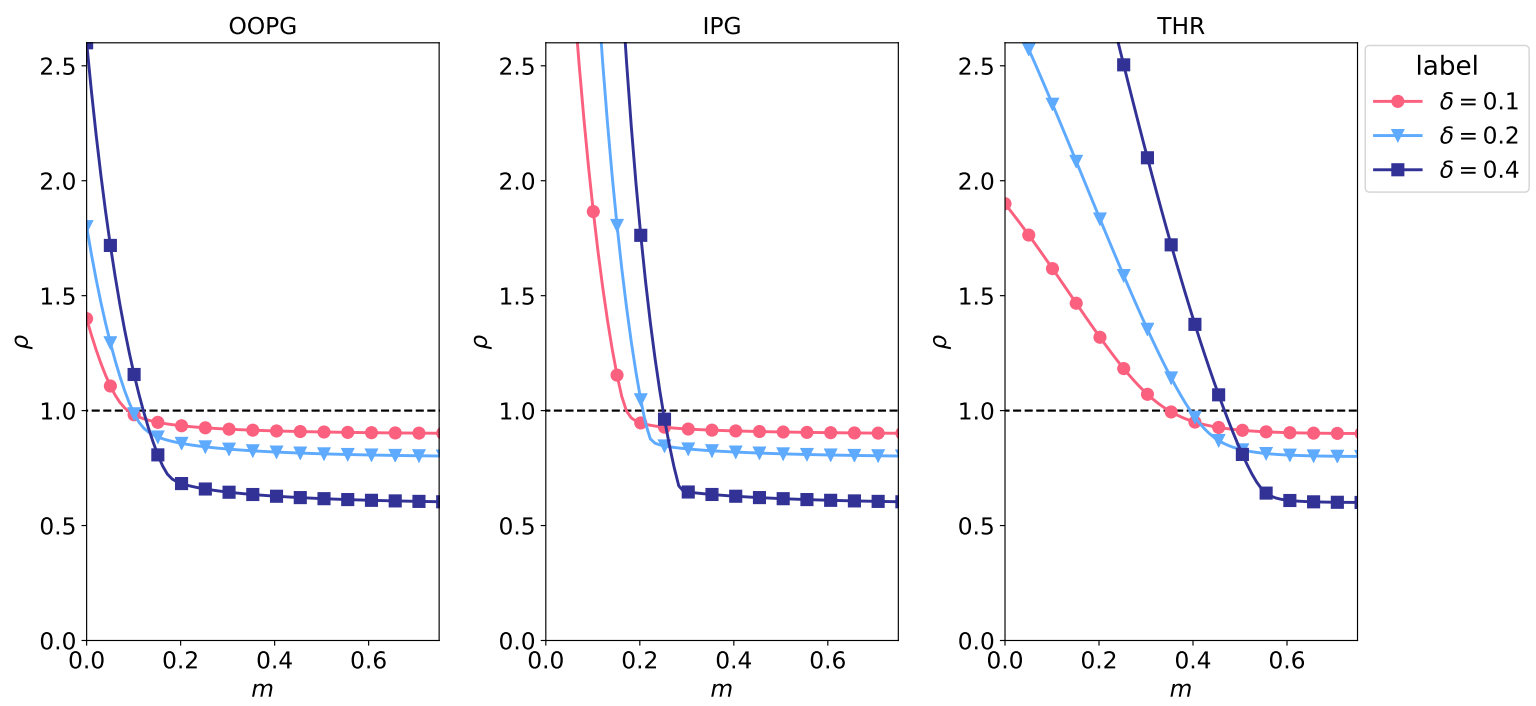

Figure 3.12: $\rho$ against different values of migration rates $(m)$ at three different selection strength $(\delta)$ levels for different public goods games. Dashed black line indicates the critical value above which altruism is viable. At each panel, a different public goods game is considered: from left to right, respectively, OOPG (Others-Only Public Goods Game), IPG (Iterated Public Goods Game) and THR (Threshold Public Goods Game). For all public goods games, we considered $n=20$. OOPG parameters: $b=5, c=1$; IPG parameters: $b=5, c=1, T=10, \theta=8$; THR parameters: $b=10$, $b^{\prime}=10, c=1, \theta=4$. 


\subsection{Discussion - when relatedness is not enough}

As introduced before, Hamilton's rule explains how to connect relatedness $(r)$ between individuals to certain social behaviors, for instance, altruism. In the original formulation, as seen in section 2.4.2, the use of Hamilton's rule is restricted to situations in which selection interactions are dyadic. A more general formulation, however, should allow $r$ to include nonlinear effects on fitness as well as effects on selection of assortment. This can be done in the TLFW framework and, in this section, we explain why it can be useful to generalize Hamilton's rule in this sense.

One of the most widely used formulations is the one from Taylor and Frank, who proposed a more straightforward way to calculate the inclusive fitness of an actor. By assuming the fitness of a focal individual depends on two variables: his own phenotype (characterized by a continuous quantity) and his social environment (defined by the average phenotype of the neighborhood), they are able to calculate the condition in which a rare mutation of the phenotype is an ESS.

The studied phenotype may be, for example, the amount of a substance that benefits nearby individuals but is costly to produce. We will denote this quantity as $y$. Following the treatment of Taylor and Frank, we would like to discover the condition under which a rare mutant could invade a monomorphic population with phenotype $y=\bar{y}$. It is assumed the rare mutation has a phenotype $y=\bar{y}+\delta$, with a small $|\delta|$.

From the definition of ESS, we know that the rare mutant $(m)$ can invade the population of wild types $(W)$ if the mutant's average fitness $\left(w^{m}\right)$ is higher than the wild type's $\left(w^{W}\right)$. Thus, to find the condition for the invasion of rare mutants, we should solve the following inequation:

$$
w^{m}-w^{W}>0
$$

The key to calculate these quantities, $w^{m}$ and $w^{W}$, is the fitness of the respective focal individual. For a wild-type individual, the calculation is very straightforward, as we can assume the social environment is composed of wild-type individuals. In this case, $w^{W}=w(y, z)=w(\bar{y}, \bar{y})$.

Now, let $X$ be a random variable of the frequency of mutants in the social environment of a mutant. Then, the average phenotype in the social environment of a mutant is: $z=(\bar{y}+\delta) X+\bar{y}(1-X)=$ $\bar{y}+\delta X$. This means that if the focal individual is the rare mutant, $w^{m}=\mathrm{E}[w(\bar{y}+\delta, \bar{y}+\delta X)]$, in which E represents the expectation of $w$ relative to $X$. Note that $X$ conveys the information 
about the assortment of mutant types in the population, which may be due to several factors, for instance, a common descent.

In the derivation of the condition for the invasion of rare mutants, Taylor and Frank expanded the fitness function $w(\bar{y}+\delta, \bar{y}+\delta X)$ into a Taylor series up to the first order and, then, applied the chain rule to calculate the derivatives of $w$ in relation to $X$ in the point $y=z=\bar{y}$. Although there was no remark about it in their derivation, this step can only be made when the partial derivatives of the fitness function $w$ exist at the analyzed point. Note that it is not strictly necessary that the function is differentiable at that point, but the function should be be well approximated by a plane.

If this condition is satisfied, then:

$$
w(\bar{y}+\delta, \bar{y}+\delta X)=w(\bar{y}, \bar{y})+\left.\frac{\partial w}{\partial y}\right|_{y=z=\bar{y}} \delta+\left.\frac{\partial w}{\partial z}\right|_{y=z=\bar{y}} \delta X
$$

Thus, using eq. (3.39) to calculate eq. (3.38), we have:

$$
\begin{aligned}
w^{m}-w^{W} & =\mathrm{E}\left[w(\bar{y}, \bar{y})+\left.\frac{\partial w}{\partial y}\right|_{y=z=\bar{y}} \delta+\left.\frac{\partial w}{\partial z}\right|_{y=z=\bar{y}} \delta X\right]-w(\bar{y}, \bar{y}) \\
& =\delta\left(\left.\frac{\partial w}{\partial y}\right|_{y=z=\bar{y}}+\left.\frac{\partial w}{\partial z}\right|_{y=z=\bar{y}} \mathrm{E}[X]\right) \\
& =\delta(-c+b r)>0
\end{aligned}
$$

From eq. (3.40), we can see $w^{m}-w^{W}>0$ takes the form of Hamilton's equation when we replaced the partial derivatives in $y$ and $z$ by $-c$ and $b$, respectively. Also, in the above expression, we replaced $\mathrm{E}(X)$ by $r$, as it can be interpreted as the average relatedness in the social environment of a mutant individual.

\subsubsection{A practical example - contingent cooperation}

Several of the behaviors displayed by humans are conditioned on the behavior of their peers. A remarkable example was studied by Schelling, who realized the dynamics of a standing ovation depended not only on the quality of the presentation, but on how individuals reacted to other individuals standing around them. Analogously, contingent cooperation strategies may also be dependent on thresholds: in the iterated public goods game, a contingent strategy could be to cooperate on the first round and then only cooperate if at least $\theta$ of the other individuals cooperated. 
Suppose individuals can be classified as type $A$ or type $N$ and are divided in groups with equal size $n$. Within each group, individuals interact with each other in such a way that, if there are at least $\theta$ individuals of type $A$ (who always bear a $\operatorname{cost} c>0$ ) inside the group, then all individuals of the group will receive a benefit $b>0$. In this situation, for a small $\delta$, an individual of type $A$ inside a group with $k$ individuals of type $A$ has fitness given by:

$$
w_{k}^{A}= \begin{cases}w_{0}-\delta c & \text { if } k<\theta \\ w_{0}+\delta(b-c) & \text { if } k \geq \theta .\end{cases}
$$

In the same group, an individual of type $N$ has fitness:

$$
w_{k}^{N}= \begin{cases}w_{0} & \text { if } k<\theta \\ w_{0}+\delta b & \text { if } k \geq \theta .\end{cases}
$$

We would like to calculate the condition in which a rare mutant type $A$ can invade a population in which type $N$ individuals are common. From eq. (3.41) and eq. (3.42), we can derive the form of $w(y, z)$ :

$$
w(y, z)= \begin{cases}w_{0}-c(y-\bar{y}) & \text { if } k<\theta \\ w_{0}-c(y-\bar{y})+b(z-\bar{y}) & \text { if } k \geq \theta\end{cases}
$$

As with the general case, we start from eq. (3.38) and should calculate $w^{m}=\mathrm{E}[w(\bar{y}+\delta, \bar{y}+\delta X)]$ and $w^{W}=w(\bar{y}, \bar{y})$. Note that this calculation is equivalent to $w^{m}=\mathrm{E}\left(w_{k}^{A}\right)$ and $w^{W}=\mathrm{E}\left(w_{k}^{N}\right)$.

Because type $N$ is much more frequent in the population, we can assume his neighbors will also be type $N$ individuals, so that definitely $k<\theta$, and we have:

$$
w^{W}=w_{0} .
$$

On the other hand, if the focal individual is of type $A$, we assume there is an increased chance that there are more type $A$ individuals inside the group and, thus, calculating $w^{m}$ is not as straightforward as when the focal individual is of type $N$ :

$$
w^{m}=\sum_{k=0}^{n} k w_{k}^{A}=w_{0}-\delta c+\delta b \operatorname{Pr}(k \geq \theta \mid A)
$$


We can see from eq. (3.45) that the calculation requires knowledge about the cumulative distribution of variable $k$ (given the focal individual is of type $A$ ) or, more precisely, about $\operatorname{Pr}(k \geq \theta \mid A)=$ $1-\operatorname{Pr}(k<\theta \mid A)$.

Note that the random variable $K$ that controls the number of type $A$ individuals inside a group corresponds to $n X$, with $X$ defined as in the derivation of the Hamilton's rule through the Taylor and Frank framework. However, since the other variables $y, z$ and $\theta$ would also be scaled by a factor $1 / n$, we merely replace $X$ by $K$ in eq. (3.39) and find the following equation:

$$
w^{m}=w_{0}+\delta[-c+b \mathrm{E}(K)]
$$

If eq. (3.46) held, considering eq. (3.45) would imply that:

$$
\operatorname{Pr}(k \geq \theta \mid A)=\mathrm{E}(K)
$$

However, this is not always true, as the value of $\operatorname{Pr}(k \geq \theta \mid A)$ depends on the distribution of $k$. Thus, in some cases, relatedness is not enough for predicting whether a rare mutant is capable of invading a monomorphic population composed of wild types.

\section{Different probability distributions can have the same mean}

Mathematically, it is clear that $\mathrm{E}(K)$ is not always the same as $\operatorname{Pr}(k \geq \theta \mid A)$. However, is it biologically relevant? Here, we intend to show an example in which the mating system influences the distribution of $k$, so that calculating the relatedness between individuals is not enough for calculating the mean fitness of a rare mutant of type $A$.

Suppose $n$ individuals are divided in $m$ males and $f$ females. In this model, one offspring has male $i$ as a father and female $j$ as a mother with probability $\mu_{i} \phi_{j}$, with $\sum_{k=1}^{m} \mu_{k}=1$ and $\sum_{k=1}^{f} \phi_{k}=1$ . In addition to these assumptions, we shall assume the same population structure and the same payoffs as the contingent cooperation model explained previously.

The transmission of traits is vertical and individuals of type $A$ are born if one of his parents is of type $A$ as well. As before, we are interested in calculating $\operatorname{Pr}(k \mid A)$ inside the group of the focal 
individual. This probability depends on $\mu_{i}$ and $\phi_{i}$ values in the following way:

$$
\operatorname{Pr}(k \mid A)=\sum_{i=1}^{m} \frac{\mu_{i}}{2} \operatorname{Bin}\left(k ; n-1, \frac{\mu_{i}}{2}\right)+\sum_{i=1}^{f} \frac{\phi_{i}}{2} \operatorname{Bin}\left(k ; n-1, \frac{\phi_{i}}{2}\right),
$$

in which $\operatorname{Bin}(k ; n, p)$ is the binomial probability distribution giving the probability of $k$ successful trials in $n$ independent Bernoulli trials, each one with probability $p$ of success.

The reasoning behind eq. (3.48) is that the probability an individual is type $A$ because of his father (mother) is $\mu_{i} / 2\left(\phi_{i} / 2\right)$. At the same time, because type $A$ is rare, all type $A$ individuals inside a group are supposed to be related, so that they have inherited type $A$ trait from the same parent and, thus, the probability that $k$ of the other $n-1$ individuals are of type $A$ is given by the probability that $k$ individuals inherit type $A$ from father $i$, Bin $\left(k ; n-1, \frac{\mu_{i}}{2}\right)$, or from mother $i$, $\operatorname{Bin}\left(k ; n-1, \frac{\phi_{i}}{2}\right)$, with $k \in[0, n-1]$.

Analogously, we can reach a formula for relatedness in this model. As type $A$ is rare, we can calculate $r$ by calculating the probability an individual is of type $A$ given that the focal individual is of type $A, \operatorname{Pr}(A \mid A)$ :

$$
r=\operatorname{Pr}(A \mid A)=\sum_{i=1}^{m} \frac{\mu_{i}^{2}}{4}+\sum_{i=1}^{f} \frac{\phi_{i}^{2}}{4}
$$

When considering mating systems, it is possible that one individual is more prone to mate than his/her peers. The degree of mating skew might be modeled by assuming that $\mu_{i}=(1-\beta) \beta^{i-1} /\left(1-\beta^{m}\right)$, where $0<\beta<1$. In the limit $\beta \rightarrow 0$, male mating is completely skewed, while in the limit $\beta \rightarrow 1$, male mating is egalitarian. Analogously, for females, $\phi_{i}=(1-\alpha) \alpha^{i-1} /\left(1-\alpha^{f}\right)$, with $0<\alpha<1$. Calculating the first term of eq. (3.49):

$$
\begin{aligned}
\sum_{i=1}^{m} \mu_{i}^{2} & =\frac{(1-\beta)^{2}}{\left(1-\beta^{m}\right)^{2}} \sum_{i=1}^{m}\left(\beta^{2}\right)^{i-1}=\frac{(1-\beta)^{2}}{\left(1-\beta^{m}\right)^{2}} \frac{\left(1-\beta^{2 m}\right)}{\left(1-\beta^{2}\right)} \\
& =\frac{(1-\beta)^{2}}{\left(1-\beta^{m}\right)^{2}} \frac{\left(1-\beta^{m}\right)\left(1+\beta^{m}\right)}{(1-\beta)(1+\beta)}=\frac{(1-\beta)\left(1+\beta^{m}\right)}{(1+\beta)\left(1-\beta^{m}\right)}
\end{aligned}
$$

Similarly, for the second term of eq. (3.49):

$$
\sum_{i=1}^{f} \phi_{i}^{2}=\frac{(1-\alpha)\left(1+\alpha^{f}\right)}{(1+\alpha)\left(1-\alpha^{f}\right)}
$$


Replacing eqs. (3.50) and (3.51) into eq. (3.49) yields an expression for $r$. If $n$ is sufficiently large (so that $m$ and $f$ are sufficiently large) this becomes:

$$
r \approx \frac{1}{4}\left(\frac{1-\beta}{1+\beta}+\frac{1-\alpha}{1+\alpha}\right)=\frac{1}{2} \frac{(1-\alpha \beta)}{(1+\alpha)(1+\beta)}
$$

In fig. 3.13, we plot eqs. (3.48) and (3.52) for a chosen set of parameters to understand the relatedness $(r)$ behavior concerning the variation of $\alpha$ and $\beta$. In the left panel, we can see that different values of $\alpha$ and $\beta$ may yield the same relatedness level. However, having the same relatedness level does not imply that the quantity $\operatorname{Pr}(k \geq \theta \mid A)$ is the same, as shown for $\theta=9$ in the right panel of the same figure.
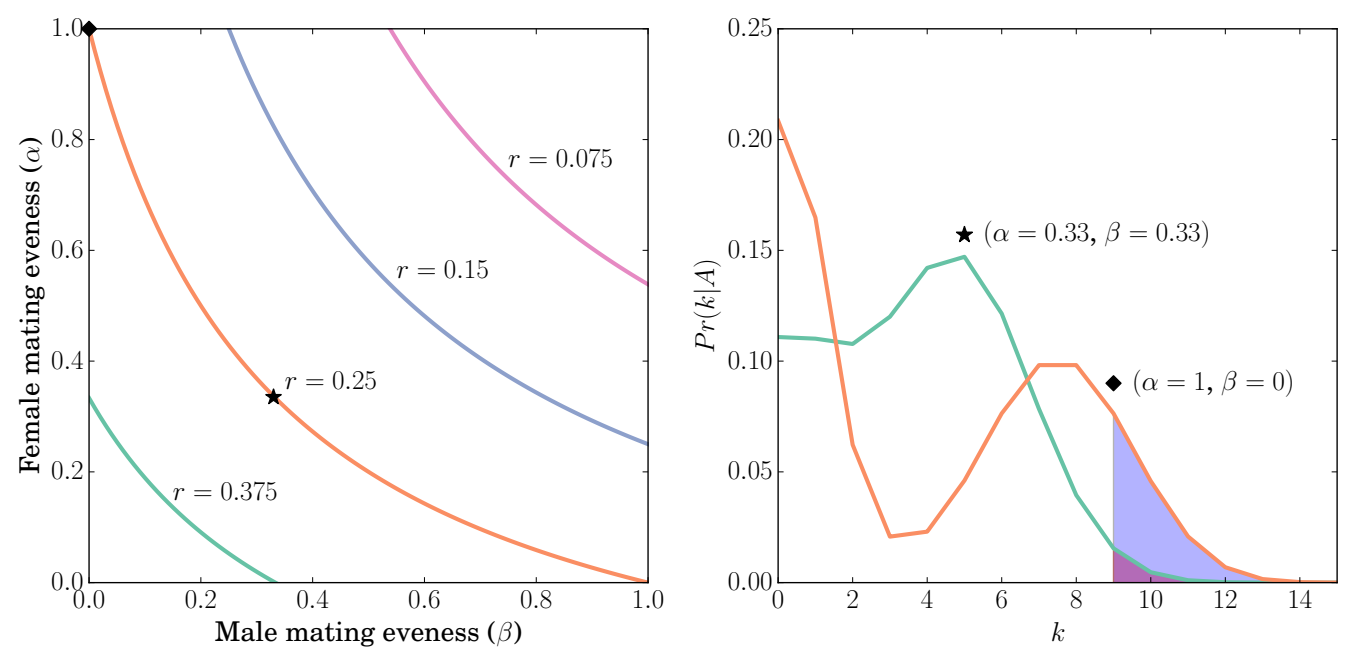

Figure 3.13: In the left panel, we see how different values of $\alpha$ and $\beta$ might lead to the same relatedness values $(r)$. In the right panel, the distribution of $k$ is plotted for two different sets of $\alpha$ and $\beta$ (represented by the star and the diamond markers), which lead to the same relatedness level, $r=0.25$. The shaded areas represent $\operatorname{Pr}(k \geq 9 \mid A)$. Parameters: $n=16, f=10$ and $m=6$.

\subsubsection{A general way of calculating the condition for the invasion of rare mutants}

The fitness function for the previous example, shown in eq. (3.43), is a non linear function in $z$, as the behavior in $z$ direction depends on the number $k$ of individuals of type $A$ inside the group. As explained before, Taylor and Frank's derivation of the condition for the invasion of rare mutants depends on the differentiability of the function $w(y, z)$, in both directions, at the point $y=z=\bar{y}$. It is easy to see that because of the threshold nature of eq. (3.43), w(y,z) is not differentiable at this point. 
For non-differentiable functions, Taylor expansion and the chain rule cannot be applied. Thus, we have to go back to the condition for the invasion of rare mutants formula:

$$
w^{m}-w^{W}=\delta \mathrm{E}\left[\frac{w(\bar{y}+\delta, \bar{y}+\delta x)-w(\bar{y}, \bar{y})}{\delta}\right]>0
$$

in which we have multiplied and divided the argument of the expectation function by $\delta$.

Since we are interested in the case where $|\delta| \ll 1$, calculating eq. (3.53) can be reduced to the problem of solving the following expression:

$$
\lim _{\delta \rightarrow 0} \frac{w(\bar{y}+\delta, \bar{y}+\delta x)-w(\bar{y}, \bar{y})}{\delta}
$$

Note that eq. (3.54) resembles the form of a directional derivative of $w(y, z)$ in the direction of a unit vector $\vec{v}$. In this case, we would multiply the expression from eq. (3.54) by $\sqrt{1+x^{2}}$ and take $\vec{v}$ as the unit vector: $\vec{v}=\left(\frac{1}{\sqrt{1+x^{2}}}, \frac{x}{\sqrt{1+x^{2}}}\right)$ at the point $y=\bar{y}$; as we are treating the case in which mutants are rare, $0<x \ll 1$, so that $\sqrt{1+x^{2}}$ is negligible. Also, note that we can write $z$ in terms of $y$, so that $w(y, z)=w(y, y x+\bar{y}(1-x))$. Hence:

$$
w^{m}-w^{W} \approx \delta \mathrm{E}[\nu(x)]
$$

where $\nu(x)=\left.\frac{d w(y, y x+\bar{y}(1-x))}{d y}\right|_{y=\bar{y}}$ and $\nu(x)$ is called marginal fitness.

Using eq. (3.55), we can then calculate condition for the invasion of rare mutants for the example of contingent cooperation as shown in box 3 .

Box 3: Condition for the invasion of rare mutants for the contingent cooperation example Using eq. (3.43) and the correspondence between $k$ and $x$, we can calculate $\nu(k)$ :

$$
\nu(k)= \begin{cases}-c & \text { if } k<\theta \\ -c+b k & \text { if } k \geq \theta\end{cases}
$$

Replacing eq. (3.56) into eq. (3.55), we have:

$$
w^{m}-w^{W}=-c+b \operatorname{Pr}(k \geq \theta \mid A)>0
$$

which is the same result we would get if we used eq. (3.45). 


\section{Chapter 4}

\section{Evolving altruism in the presence of intergroup conflicts}

In this chapter, we study the emergence of altruism in the presence of intergroup conflicts under a weak selection regime, by using a model based on a two-level selection model. Our results show conditions for the viability of altruistic traits in the context of hunter-gatherers societies.

\subsection{Related work and motivation}

Archaeological and ethnographic studies $[4,50,54,55]$ provide evidence that hunter-gatherer populations might have engaged in frequent intergroup conflicts, although it is not clear at what extent hunter gatherers were violent.

Altruism can be defined as the strictiest version of cooperation, when an actor incurs a cost to benefit the recipient exclusively. Several theories have been proposed to explain its evolution but, so far, there has been no definitive answer to how altruism could have emerged in human populations. Most theories involve kin selection, indirect and direct reciprocity and multilevel selection [57].

Previously, Bowles $[16,17]$ modeled and discussed the emergence of altruism in this scenario, by using the Price equation. The Price equation [78] is an expression for the difference of the frequency of a type of a particle or individual between two subsequent generations. It is simple, recursive and separates clearly the effects of inter and intragroup selection [42]. Recently, there has been a discussion of whether the use of Price equation for evolutionary models is correct or not. Van Veelen 
et al. [100] claim that some models based on the Price equation are not dynamically sufficient, i.e., the models use equations which does not account for changes across time.

One of the most important consequences of the Price equation is the Hamilton rule, which is a condition for the proliferation of altruism. However, it is known that the simplest form of the Hamilton rule does not hold for any scenario.

As presented in the previous chapter, an alternative framework to model the evolution of altruism was introduced by Schonmann et al. [82, 83]. They consider two levels of selection with migration in a structured population (a two-level Fisher-Wright model). Their approach generates a simple rule, which predicts the conditions in which altruism can survive. This model also recovers the original rule, the Hamilton rule, for the cases in which fitness functions are linear or, equivalently, interactions are dyadic.

\subsection{The model}

We consider a large population with an infinitely large number of groups of fixed size $n$ in which individuals are randomly distributed. Each individual is labeled as an altruist $(A)$ or as a native ( $N$, non-altruist). Since we would like to study the proliferation of altruists in the population, we classify the groups according to their number of altruists. We define that a group will be of type $k$ if it has exactly $k$ altruists and, consequently, $n-k$ non-altruists.

This is a three-staged model (see fig. 4.1). In the first stage, groups may engage in conflicts between each other, and this stage is called intergroup selection. Next, there is the stage of intragroup selection, in which individuals interact inside their groups, and reproduce according to their innergroup fitness. Finally, there is migration of individuals among the groups, which decreases the relatedness level of individuals in the same group.

Each generation in our model can be characterized by the product of two matrices. One of them, the matrix $S$, represents the intergroup and intragroup selection processes. The other matrix, $M$, captures the migration process. We assume the dynamics for the number of altruists eq. (4.1) is a Markov process. Thus, by providing an initial distribution of altruists among groups, we are able to track the expected frequency of altruists in the population at a given generation.

$$
N(t+1)=N(t) S M
$$




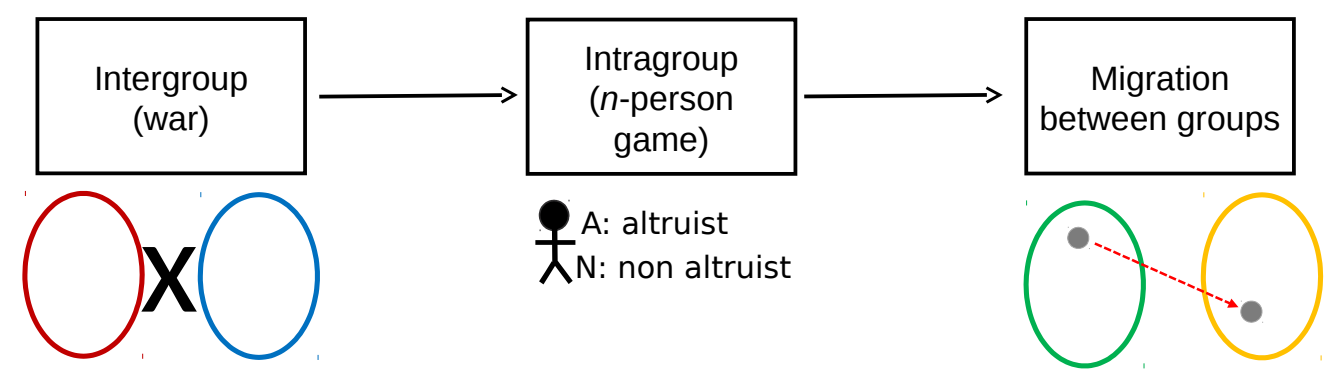

Figure 4.1: Model scheme. First, there is war between groups. Secondly, intragroup selection takes place and individuals reproduce. Finally, individuals are allowed to migrate to other groups.

in which $N(t)=\left(N_{1}(t), \ldots, N_{n}(t)\right)$ and $N_{k}(t)$ is the average number of groups of type $k$.

In the intergroup selection stage, groups can engage in conflicts between each other with probability $\beta$. The likelihood of winning a contest depends on the number of altruists and also on parameter $\alpha$, the benefit of altruists in conflicts, which measures the influence of altruists in the war outcome. A scheme of the intergroup selection process is shown in fig. 4.2 .

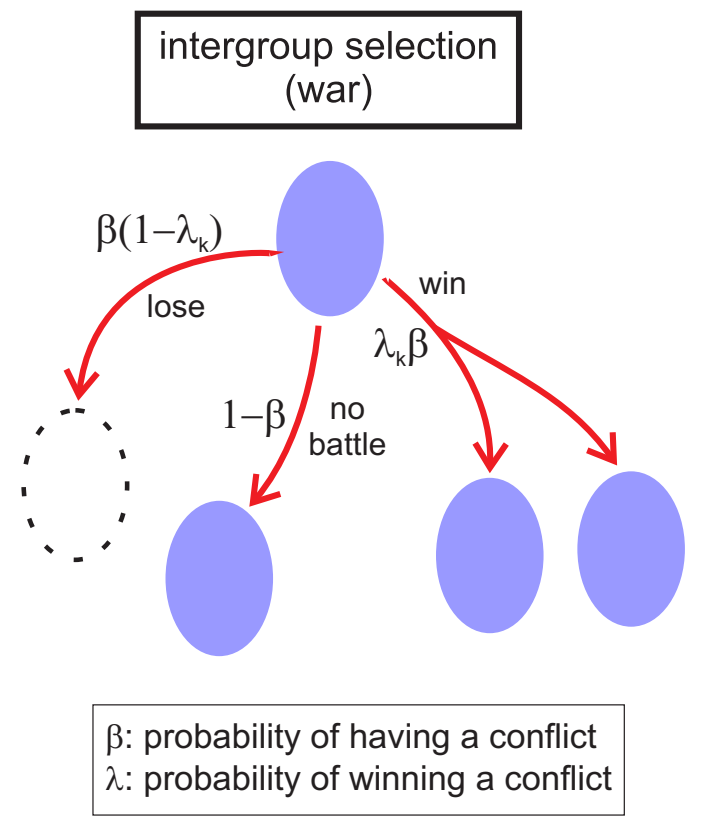

Figure 4.2: Intergroup selection in our model. Groups engage in war with probability $\beta$ and can win or lose the war according to a function $\lambda_{k}$ that depends on the number of altruists $k$ in the group. Three outcomes are possible from this process: either the group does not fight the war and keeps its deme; or it fights the war and may go to extinction or reproduce twice, at its own deme and at the opponent deme.

We consider $\alpha \in(0,1)$, and the more altruists one group has, the more likely it is to win the war, as shown in eq. (4.2) for the likelihood of winning the war. If the number of altruists of the focal 
group is zero, then, there is equal likelihood of winning or losing the war ${ }^{1}$.

$$
\lambda_{k}=\frac{1}{2}+\alpha \frac{k}{2 n}
$$

In this stage, a group may go extinct (size 0), if it loses the war, or survive, and be the owner of two demes (size 2), if it wins the war. If the group had not engaged in any contest, it will keep occupying its own deme (size 1). Thus, $\bar{s}$ is the average size of a group, which depends on the probability $\beta$ of engaging in war and on the likelihood $\lambda_{k}$ of winning the war:

$$
\bar{s}_{k}=1-\beta+2 \beta \lambda_{k}
$$

By the end of intergroup selection stage, the intragroup selection stage begins, in which reproduction takes place in all groups that have survived. We assume generations do not overlap. Hence, individuals die right after giving birth to their offspring. The offspring occupies the group demes (each one with capacity for $n$ individuals).

The reproduction process occurs according to the inner-group fitness of individuals (eq. (4.4), which is the result from interactions between individuals inside their groups. We assume weak selection, so that all individuals receive an equal baseline inner-group fitness, 1 , but every altruist bears a cost $c$, while non-altruists do not have any associated cost.

$$
\begin{aligned}
& v^{A}=1-c \\
& v^{N}=1,
\end{aligned}
$$

The proportion of altruists in the child population for each deme of each group will be the average inner-group fitness of that group members and, hence, will depend on the number of altruists inside the group, $k$. Thus, on average, each deme will have $\bar{v}_{k}$ altruists:

$$
\bar{v}_{k}=\frac{k v^{A}+(n-k) v^{N}}{n}=1-c \frac{k}{n} .
$$

Since we assume the group size, $n$, remains constant, the reproduction process can be seen as a $n$ trials sampling without replacement, as if $n$ children were choosing their parents from the previous

\footnotetext{
${ }^{1}$ Although there is some controversy around defining what counts as war in a hunter-gatherer society [50, 38, 56, 51], from this point on, we will, for the sake of simplicity, refer to wars and conflicts interchangeably.
} 
generation. In this process, a child can choose an altruist parent with probability $p_{k}^{A}=k v^{A} / n \bar{v}_{k}$ or a non-altruist parent with probability $p_{k}^{N}=1-p_{k}^{A}$. Hence, a group with $k$ altruists (among $n$ individuals) will become a group of $l$ altruists after the reproduction process with probability $q_{k l}=\mathrm{P}\left(\operatorname{Bin}\left(n, p_{k}^{A}\right)=l\right)$, with $\operatorname{Bin}\left(n, p_{k}^{A}\right)$ representing a random variable following a Binomial distribution with parameters $n$ and $p_{k}^{A}$.

The two selection processes introduced above, war and reproduction, are enclosed in the matrix $S=\left(S_{k l}\right)_{k, l=0,1, \ldots, n}$. Each element $S_{k l}$ of matrix $S$ is the average number of groups of type $l$ created from groups of type $k$, and is given by eq. (4.6). From the equation, we can see that $S_{k l}$ is the product of the average size of a group with $k$ altruists due to intergroup selection $\left(\bar{s}_{k}\right)$ with the probability that a group with $k$ altruists have $l$ altruists due to intragroup selection $\left(q_{k l}\right)$.

$$
S_{k l}=\bar{s}_{k} q_{k l}=\bar{s}_{k} \mathrm{P}\left[\operatorname{Bin}\left(n, \frac{k v^{A}}{n \bar{v}_{k}}\right)=l\right]
$$

It is clear that, in our model, individuals' fitness are affected by both processes describe above: the war between groups and the interactions inside groups (inner-group fitness). Since fitness is defined as the number of produced offspring over the number of parents for a given individual type, we can use matrix $S$ to calculate it. Then, after getting the average number of offspring for each type of group, we obtain the following expression for the average fitness of altruists, i.e., the expected value of $w_{k}^{A}, \mathrm{E}\left[w_{k}^{A}\right]:$

$$
\mathrm{E}\left[w_{k}^{A}\right]=\frac{\sum_{k=0}^{n} \sum_{l=0}^{n} l S_{k l} \nu_{k}}{\sum_{k=0}^{n} k \nu_{k}}
$$

in which $\nu_{k}$ corresponds to the frequency of type $k$ in the population, $k=1,2, \ldots, n$.

In the last stage of our model, finally, altruists and non-altruists migrate among groups at rate $m$, indistinctly. As we are restricting ourselves to the weak selection regime, we can consider a simplified version of this process, following Schonmann et al. [83]. This way, we can see migration as a two-stage process, as explained in section 3.2.

Thus, the matrix M can be decomposed as a sum of two other matrices, as shown in eq. (4.8). First, individuals migrate with rate $m$, so that altruists will stay in its original group with probability $1-m$ (see eq. (4.9)). And, second, due to the assumption of an infinitely large numbers of groups, and a few number of altruists in the population, migrants arrive at their new groups with the 
condition that only groups with non-altruists can receive migrant altruists (eq. (4.10)).

$$
M=A+B
$$

$$
A_{k^{\prime}, k}=\mathrm{P}\left(\operatorname{Bin}\left(k^{\prime}, 1-m\right)=k^{\prime \prime}\right)
$$

$$
B_{k^{\prime}, k^{\prime \prime}}= \begin{cases}=m k^{\prime}, & \text { if } k "=1 \\ =0, & \text { if } k " \neq 1\end{cases}
$$

It is possible to think about this model as a multitype branching process [83], since we are counting the numbers of groups from types 1 to $n$. If no group survives, this means there is no altruist in the population. Hence, from the theory of multitype branching processes, we find a condition for the viability of altruism, given by:

$$
\rho(m)>1,
$$

in which $\rho(m)$ is the dominant eigenvalue of matrix $R$, which is a reduced matrix from the product $S M$, without the first row and column, as explained in section 3.2.

Furthermore, the stationary distribution of altruists in the population, is given by the left eigenvector, $\nu$, of the matrix $R$. The existence of a dominant eigenvalue $\rho(m)$, dependent on the migration level $m$, is guaranteed because matrix $R$ has only non-negative entries, so that we can apply the Perron-Frobenius Theorem [64].

\subsubsection{Deriving an analytical condition for the emergence of altruism}

In our model, there was no explicit expression for the fitness functions of altruists and non-altruists, but by using the assumptions in our model, it is possible to calculate them from eq. (4.12). Fitness is defined as the number of produced offspring over the number of parents for a given individual type. In our model, we can calculate the average number of offspring for each type of group, so that we can obtain the following expression for the average fitness of altruists, i.e., the expected value of the fitness of altruists, $\mathrm{E}\left[w_{k}^{A}\right]$ : 


$$
\mathrm{E}\left[w_{k}^{A}\right]=\frac{\sum_{k=0}^{n} \sum_{l=0}^{n} l S_{k l} \nu_{k}}{\sum_{k=0}^{n} k \nu_{k}},
$$

in which $\nu_{k}$ corresponds to the frequency of type $k$ in the population, $k=1,2, \ldots, n$.

Replacing $S_{k l}$ from eq. (4.6), we have:

$$
\mathrm{E}\left[w_{k}^{A}\right]=\frac{\sum_{k=0}^{n} \nu_{k}\left(1+2 \beta \alpha \frac{k}{n}\right) \sum_{l=0}^{n} l \mathrm{P}\left(\operatorname{Bin}\left(n, p_{k}^{A}\right)=l\right)}{\sum_{k=0}^{n} k \nu_{k}},
$$

in which $p_{k}^{A}=\frac{k v^{A}}{n \overline{v_{k}}}=\frac{k(1-c)}{n(1-c k / n)}$.

The internal sum is easily calculated, since:

$$
\sum_{l=0}^{n} l \mathrm{P}\left(\operatorname{Bin}\left(n, p_{k}^{A}\right)=l\right)=\mathrm{E}\left[\operatorname{Bin}\left(n, p_{k}^{A}\right)=l\right]=n p_{k}^{A}
$$

Thus, from eq. (4.13), we have the following expression for $\mathrm{E}\left[w_{k}^{A}\right]$ :

$$
\begin{aligned}
\mathrm{E}\left[w_{k}^{A}\right] & =\frac{\sum_{k=0}^{n} \nu_{k}\left(1+2 \beta \alpha \frac{k}{n}\right) n \frac{k(1-c)}{n(1-c k / n)}}{\sum_{k=0}^{n} k \nu_{k}} \\
& =\frac{\sum_{k=0}^{n} k \nu_{k}\left(1+2 \alpha \beta \frac{k}{n}\right) \frac{(1-c)}{(1-c k / n)}}{\sum_{k=0}^{n} k \nu_{k}} .
\end{aligned}
$$

But, from the definition of the expectation of a function of a random variable, $\mathrm{E}\left[w_{k}^{A}\right]$ can be written as:

$$
\mathrm{E}\left[w_{k}^{A}\right]=\frac{\sum_{k=0}^{n} k \nu_{k} w_{k}^{A}}{\sum_{k=0}^{n} k \nu_{k}} .
$$

By comparing eqs. (4.15) and (4.16), we can see that the fitness of altruists is actually a simple expression, given by eq. (4.17).

$$
w_{k}^{A}=\frac{(1-c)(1+2 \alpha \beta k / n)}{1-c k / n}
$$


We use the expressions for the fitness of altruists and non-altruists to derive an analytical expression for the viability of altruism, when altruism is rare in the population and when certain conditions for the parameters are observed.

To find the condition for the viability of altruists, we need to compare the average fitness of altruists with the average fitness of non-altruists. The only way altruists can emerge is if their average fitness is larger than the average fitness of the non-altruists, when there are no altruists. Thus, the condition for the emergence of altruism is given by:

$$
\mathrm{E}\left[w_{k}^{A}\right]>\mathrm{E}\left[w_{0}^{N}\right]
$$

and is equivalent to eq. (4.11).

The expression for the fitness of non-altruists, when there are no altruists, is straight from $v^{N}$ and is given by eq. (4.19).

$$
\mathrm{E}\left[w_{0}^{N}\right]=w_{0}^{N}=1
$$

As shown above, the expression for the fitness of altruists is given by eq. (4.17). Thus, the average fitness of altruists can be represented by eq. (4.20).

$$
\mathrm{E}\left[w_{k}^{A}\right]=(1-c) \mathrm{E}\left[\frac{1+2 \alpha \beta k / n}{1-c k / n}\right]
$$

We applied a Taylor expansion to the function $\left(\frac{1+2 \alpha \beta k / n}{1-c k / n}\right)$ around $c=0$. After calculating the expectation of the resulting expression, we inserted it in the eq. (4.18), obtaining a condition for the relatedness coefficient $R$ (eq. (4.21), under the assumptions of $n \rightarrow \infty$ and $m \rightarrow 0$.

$$
R>\frac{-\alpha \beta(1-c)+\sqrt{\alpha^{2} \beta^{2}(1-c)^{2}+c+2 \alpha \beta(1+c) c}}{c+2 \alpha \beta(1+c)}
$$

From eq. (4.16), we see that $\mathrm{E}\left[w_{k}^{A}\right]=\sum_{k=0}^{n} k w_{k}^{A}$. We can approximate this sum by an integral in the interval $[0,1]$, by doing a change of variable $x=\frac{k}{n}$, considering we have a sufficiently large $n$, following [24].

$$
\mathrm{E}\left[w_{k}^{A}\right] \approx \int_{0}^{1} w_{k}^{A} \operatorname{Beta}(x \mid 1,2 n m) d x
$$


Since all parameters are between 0 and 1 , this integral can be solved in terms of hypergeometric functions ${ }_{2} \mathrm{~F}_{1}$, leading to eq. (4.23).

$$
\mathrm{E}\left[w_{k}^{A}\right]=(1-c)_{2} \mathrm{~F}_{1}(1,1 ; 2 n m+1 ; c)-\frac{2 \alpha \beta\left(-2 n m-1+2 n m_{2} \mathrm{~F}_{1}(1,1,2 n m+2)\right)}{2 n m+1}
$$

\subsection{The agent-based simulation}

We performed an agent-based simulation based on the description of the model. Analogously to the theoretical model presented previously, in the agent-based simulation, for each period, there are three stages: (i) the intergroup selection stage, in which there can be conflicts between groups; (ii) the intragroup selection stage, in which individuals reproduce; and (iii) the migration stage.

In the first stage, groups can engage in conflicts between each other with some fixed probability, $\beta$. This means that with probability $\beta$, one group will be paired with another, and with probability $1-\beta$, it will not be paired with any group.

Let the focal group be a group of type $k$, while the non-focal group is a group of type $j$. The focal group will win or lose the contest, with probability $\lambda_{k j}$, which depends on the number of altruists $k$ and $j$, on the group size $n$ and on the benefits of altruists in conflicts, $\alpha$. This probability is different from the one we used in the mathematical model from the main text, in the sense that now we are able to calculate it using the number of altruists in both groups involved in the contest. In the model of the main text, we used a simplified equation, which assumed the non-focal group had no altruists $(j=0)$. For the simulation, we removed this assumption and used eq. (4.24) that depends on both $k$ and $j$, following Bowles [17].

$$
\lambda_{k j}=\frac{e^{4 \alpha \frac{k}{n}}}{e^{4 \alpha \frac{k}{n}}+e^{4 \alpha \frac{j}{n}}}
$$

There are three possible outcomes from the intergroup selection stage: (i) the group is paired with another and it wins the contest; or (ii) the group is paired with another and it loses the contest; or (iii) the group is not paired with any group.

Next, in the intragroup selection stage, individuals reproduce according to the inner-group fitness. The inner-group fitness are the same as the ones calculated before, which are derived from a simple 
function that is dependent on the type of the individual (altruist or non-altruist) and on the number of altruists inside the group of this individual.

Group reproduction follows fig. 4.2: (i) if the group has won the contest, it reproduces twice (which emulates the occupation of the loser deme); (ii) if the group has lost the contest, it becomes extinct (no reproduction); and (iii) if the group has not engaged in any contest, it reproduces repopulating its own deme. In the agent-based simulation, we still keep the assumption that generations do not overlap.

Lastly, in the migration stage, individuals migrate between groups with some fixed probability $m$. Group sizes remain constant, even after migration, so that individuals are selected to migrate with probability $m$, leaving a vacant spot inside their groups, and then, randomly distributed among all the vacant spots left by migrants. Here there is no constraint on the number of altruists of the group to which an altruist will migrate.

In summary, it is possible to see that some simplifications taken in the theoretical model were dropped in the agent-based simulation. These simplifications concerned the low frequency of altruists in the population, so that we expect the agent-based simulation yields a closer description of reality.

\subsection{Results}

From eq. (4.11), we calculate the critical migration rate necessary for the viability of altruism. The viability condition from eq. (4.11) will be referred as the eigenvalue method and it will be compared with our analytical approaches to find an expression for the viability condition. Further, we compare the results from the eigenvalue method with the results from an stochastic simulation of the model to check whether the theoretical analysis was a good approximation of the model. Finally, we show the results for sets of parameters which made sense in the context of hunter-gatherer societies.

\subsubsection{Comparing results from analytical derivation}

We obtained three different ways for analyzing altruism viability from our model. Although all of them are equivalent to the condition from eq. (4.18) for the spread of altruism, each one of them was built using a different method. 
One of them is the eigenvalue method [83], described above, in which we calculate the selection and migration matrices and check if the dominant eigenvalue of the reduced matrix from the dot product of these matrices is larger than one (eq. (4.11)). The other condition is given by the eq. (4.18) and requires the calculation of the fitness functions for altruists and non-altruists. To calculate that, we assumed $m \rightarrow 0$ and $n>>1$ and we used two different methods: (i) a Taylor expansion around $c=0$, which we will call small $c$, and (ii) a direct integral calculation using results from the equilibrium distribution of types in the population (approximation). We compare these three methods to see if they predict viability of altruism for the same set of parameters.

In fig. 4.3, we show how critical probability of war occurrence $\beta$ varies in relation to $\alpha$, which is the influence of altruists in the probability of winning a war, for two different values of migration rates, $m=0.1$ and $m=0.5$.

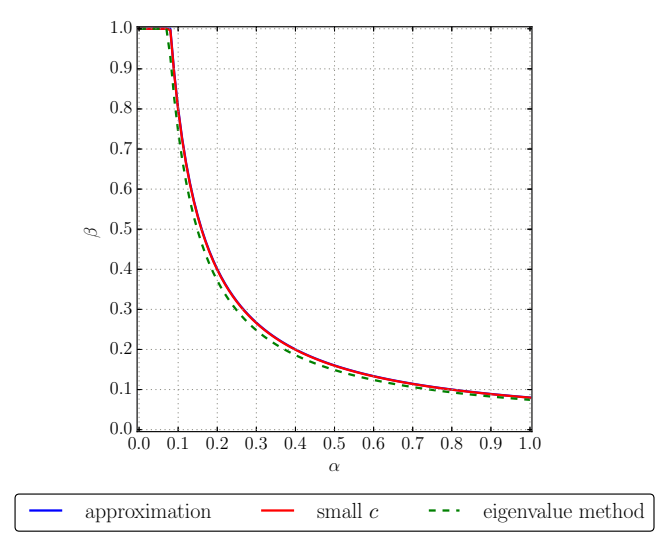

(a) $m=0.1$

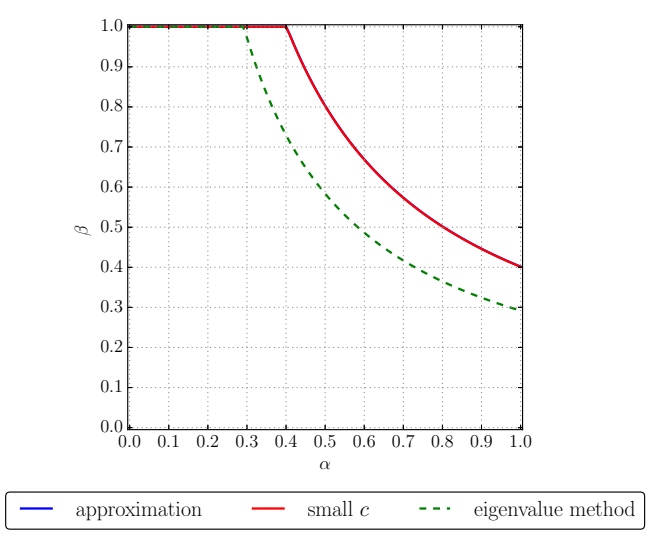

(b) $m=0.5$

Figure 4.3: Critical probability of war when $c=0.03, n=26$ for different approaches of getting the viability condition.

From these results, we observe that approximation and small $c$ curves are almost coincident for both values of $m$. However, the eigenvalue method curve is lower than the other curves, with a considerable difference in fig. $4.3 \mathrm{~b}$. This result is expected because, when deriving the analytical results, we assumed $m \rightarrow 0$ and weak selection, which in our model is translated into small values of $c, \alpha$ and $\beta$. This difference shows that the eigenvalue method predicts a more optimistic scenario than the analytical approximations.

\subsubsection{Using realistic parameter values}

In order to link our results with the context of hunter-gatherer societies, we use a range of parameters from the estimates provided by Bowles [16]. We consider the range of cost to be between 0 and 
0.08 and the range of probability of conflicts to be between 0 and, approximately, 0.9 . The blue area in both panels in fig. 4.4 represents these two ranges.

In this figure, the relation between the critical cost and $\beta$, the probability of engaging in war, is depicted for different levels of benefits of altruists in conflicts $(\alpha)$, for two different values of relatedness. In both cases, we see that if $\alpha$ is higher, altruism is viable for a larger set of values of $c$ an $\beta$.

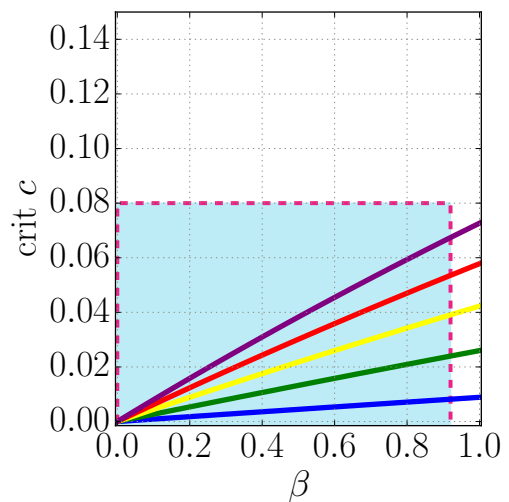

(a) $r=7 \%$

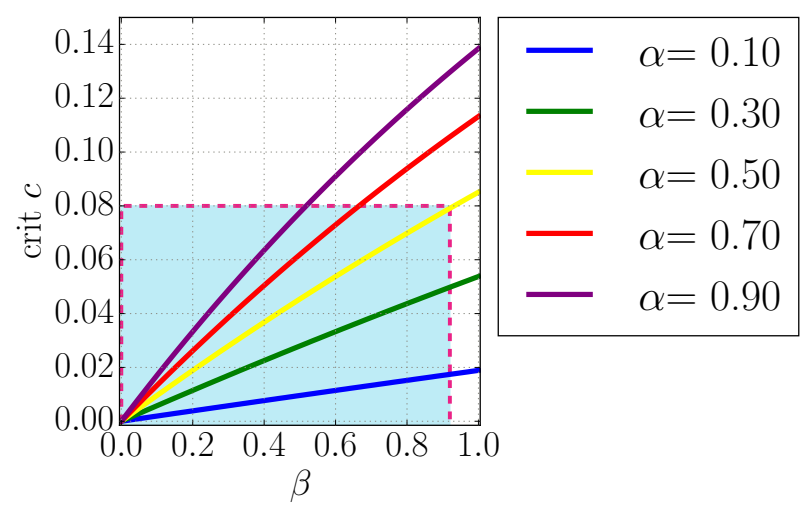

(b) $r=15 \%$

Figure 4.4: Critical cost versus probability of war for different levels of influence of altruists in the war outcome when migration is equivalent to $r=7 \%$ and $r=15 \%$ and number of individuals in the group $n=26$. Blue area represents the set of parameters which can be considered realistic.

Although a higher relatedness helps altruism to be viable in the population, we can see that a high level of intergroup conflicts and of benefits of altruists in conflicts $(\alpha)$ might be able to compensate a lower relatedness, depending on the cost. In the left panel of fig. 4.4, when the relatedness is $7 \%$, for instance, if $\beta=0.8$ and $\alpha=0.7$, the cost of being an altruist could be up to slightly more than 0.04 .

\subsubsection{Comparing simulation results with the eigenvalue method}

We compared the results obtained from the eigenvalue method from the stochastic simulation of the model. As mentioned in section 4.3, the simulation description is almost identical to the mathematical model description. The only differences are that we drop the simplifications used to make the model more tractable analytically (namely, the simplification of $\lambda_{k}$, the probability of war, and of the migration process) and we allow for mutations to occur at a rate of $10^{-4}$.

In fig. 4.5, simulation results are compared with the results obtained from the eigenvalue method for different values of $c(c=0.03$ and $c=0.15)$ and $\alpha(\alpha=0.1$ and $\alpha=0.3)$. Each point corresponds 
to the result of running 20 simulations, starting with no altruists in the population. We tracked the frequency of altruists in the populations and we considered that a viable set of parameters is the one in which at least one of the 20 simulations ended up with more than $90 \%$ of altruists in the population.

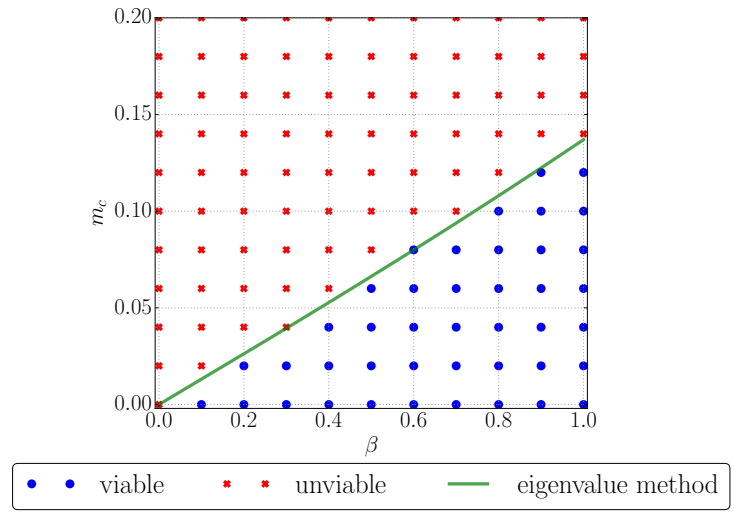

(a) $c=0.03 ; \alpha=0.1$

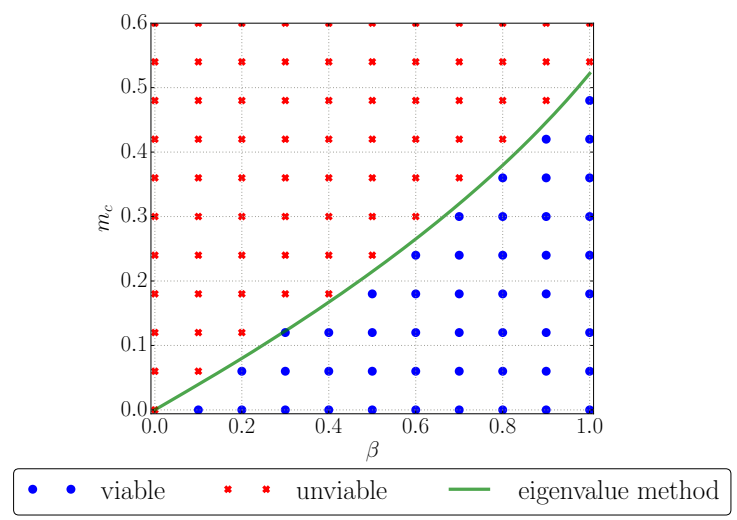

(c) $c=0.03 ; \alpha=0.3$

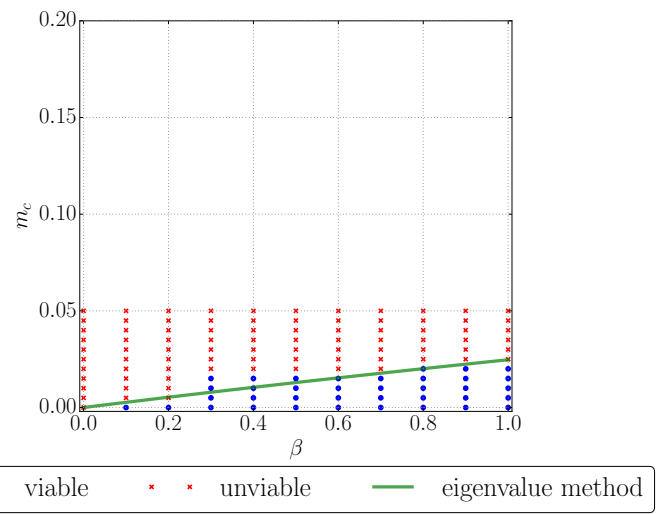

(b) $c=0.15 ; \alpha=0.1$

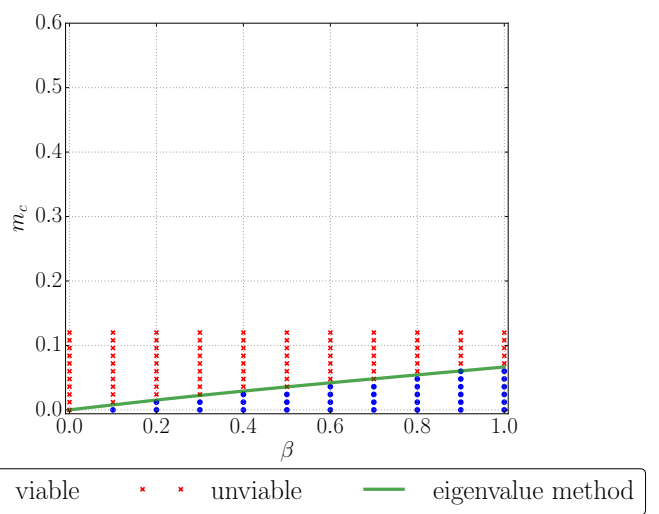

(d) $c=0.15 ; \alpha=0.3$

Figure 4.5: Comparison between simulation results and the eigenvalue method for critical migration rate $\left(m_{c}\right)$ versus probability of war $(\beta)$, when $n=26$ and for two different values of $\alpha: \alpha=0.1$ (top figures) and $\alpha=0.3$ (bottom figures). Simulation parameters: $N=5000$ (number of groups), $\mu=0.0001$ (mutation rate) and $\max _{\mathrm{IT}}=10000$ (maximum number of iterations).

These results show that our simulation agrees with the critical migration rate curve obtained from the eigenvalue method, our theoretical predictions from the model.

\subsection{Discussion}

In this chapter, we have shown that the existence of conflicts between groups could have had an important role in spreading altruism in human populations. Although in our model we did not specify the mechanism through which an altruist benefits his group in a war, it is implied that this is done through self-sacrifice: we believe altruists are more willing to risk themselves by taking an 
aggressive approach towards its enemies. In this context, we might say that an altruist could have been the one who had possessed a "warrior gene".

Indeed, the association of a gene (low activity monoamine oxidase A, MAOA-L) with aggressive behavior $[26,60]$ has been raising attention, from both scientific community and media, to the genetical roots of human violence. This gene has been referred as the "warrior gene", because of its implications for war, and it is also present in Old World monkeys. Its survival in human genome until the present day may indicate it has undergone a positive selection event $[39,6]$.

Since overly aggressive behavior would be disadvantageous, it is safe to assume some mechanism promoted the balance of different variants of MAOA in the population. One study has linked altruistic punishment with warrior gene presence, by conducting behavioral experiments [62]. Further studies could cast light on the question whether warrior gene and self-sacrifice behavior are also linked.

When building this model, our goal was to investigate the viability of altruism in a setting that included conflicts between groups. We assumed the process of intergroup conflicts could be modeled analogously to group reproduction. Thus, since altruism occurs at the level of individuals and conflicts occur at the level of groups, this clearly can be characterized as a two-level selection process.

Group selection has remained a controversial theme for several decades and many authors have tried to reach a consensus by exposing weakness of one viewpoint while promoting another viewpoint $[61,65,75,37]$. Although there has been a claim that group selection and inclusive fitness theory always yield the same results [59] and, hence, are equivalent, this is not the only opinion in the field $[99,41]$.

Another controversial aspect of group selection has been the formal mathematical models used to address it. Many of the models built to explain social evolution are based on the Price equation. However, usually they are not dynamically sufficient, i.e., they offer an explanation based on a static image of the analyzed process. Some researchers have tried to introduce more general frameworks [75, 83, 91, 37], covering one flaw or another pointed by their peers, including the dynamically insufficiency property mentioned above. However, none of these frameworks are widespread yet.

One of the highlights of this chapter was the derivation of a viability condition, when selection is weak, for the emergence of altruism when intergroup conflicts are frequent. This rule represents 
the relation between relatedness and the parameters of our model - the influence of altruists in the war outcome, the frequency of conflicts and the migration rate among groups. Our simulation confirmed the results of the theoretical model, which supports the derived analytical conditions, under weak selection. 


\section{Chapter 5}

\section{Evolving language}

In this chapter, we discuss the motivations behind the study of language evolution, and we describe the model we created to investigate the problem of the survival of a dialect, when a powerful standard language exists.

\subsection{Related work and motivation}

Language diversity has been declining for several decades, which is an example of a more general phenomena: the loss of cultural diversity due to globalization. According to the UNESCO Atlas of the World's Language in Danger [68], many languages are currently in danger of extinction in the world, particularly, in South America and Africa. This situation has no precedents in human history, and it is hard to understand the implications of losing thousands of languages in a short range of time. Since in many cases there are no written records of the endangered or already extinct language, there may be no possibility of recovery from this major cultural diversity loss.

Language is an example of cultural trait, so that the dynamics of language diversity is a good target for studying it by using evolutionary dynamics techniques. Szathmáry and Smith [93] identified the appearance of languages as one of the major transitions in evolution. Language provides an opportunity for cultural evolution, which is also a focus of evolutionary biology (e.g. [28] and [21]). Concerning language, Nowak and Krakauer [71] formalized the process of the emergence of a language, using a type of game known as a signaling game. This explains how words and grammar can evolve from a protolanguage of signals. 
Abrams and Strogatz [2] devised a simple model for the competition of two languages. Their model consists of a single differential equation which describes the dynamics of the number of speakers of the two languages, considering there are no bilinguals in the population. They showed one of the two languages will eventually become extinct. More recent works $[66,67]$ have extended the framework provided by Abrams and Strogratz, by incorporating the possibility of bilinguals arising in the population. Mira et al. [67] have reached the conclusion that when the competing languages are sufficiently similar to each other, it is possible to achieve coexistence, through the emergence of a stable bilingual group of individuals.

To study the factors controlling the language diversity, we study the dynamics of dialects of a single language. We here consider the situation in which the dialect difference is not very extensive: speakers of different dialects can communicate with each other without much difficulty, although different dialects can be identified immediately from differences in accent, terminology, and expressions. For instance, this can be seen in dialect differences in contemporary Japan.

Amano et al. [5] identified the high economic growth of a region as a key driver of language extinction, by associating GDP (Gross Domestic Product) with a rapid decline of language speakers. This result could help to explain the case of dialects, in which the existence of a national standard language usually drives individuals to drop their local dialects. This severe decline of speakers may be related to the fact that individuals prefer to speak more powerful languages. The rise of a dialect as a national language has happened in history many times, exemplified by Tokyo dialect (Japanese), Paris dialect (French) and Athens dialect (Greek) [46]. The standardization process of a language may cause other dialects to be perceived as a lower-class or rural speech [47]. This endangers the survival of different dialects; in the case of Japan, it was believed that all dialects would disappear in some decades [48].

Although the standard language is adopted widely in public situations, many dialects are still used in more intimate communications, inside family or between close friends. Thus, the use of different dialects in different situations may be an important factor to maintain the dialect diversity. Sekiguchi and Nakamaru $[86,87]$ studied a mathematical model to explain how oblique transmission affects the inconsistency of behavior and attitude, which could be related to the use one language in public while keeping another language restrict to interactions between family and friends.

Spatial structure of the population may also act to maintain the dialect diversity. Axelrod [10] focused on the factors that promote the diversity of social behaviors, finding that when two culturally 
different sites interact, stable regions of uniform cultures may appear. This is consistent with the existence of linguistic areas, when each area has speakers with slightly distinct linguistic features that may cause the edges of these linguistic areas to be completely different from each other [29]. Since language is a tool for communication between multiple people, it is quite natural to consider it would be beneficial if people adopted the same accents or expressions and, indeed, after many years of interaction, people tend to adopt the dialect of the local community to which they belong.

To build our model, we focus on the survival of a local dialect when competing against a national standard language, with the latter providing an advantage in the occupational and economic contexts. In the model, we assume that individuals may use different dialects, in response to two different situations: they may use the national language in "public situations" such as in a formal workplace, whilst they may use a local dialect "in private situations" such as in family or close friend meetings. We consider the choice of a dialect is guided by the difference in "attractiveness" of the two dialects, which reflects all the differences in the usefulness of adopting those dialects, including economic and prestige differences. In addition, we consider that the attractiveness of a dialect may change with the relative frequency of the dialect used in the local population. Specifically, we assume that each player follows a stochastic evolutionary process similar to a continuous-time Moran process, in which each player at random times adopt a dialect spoken by members of the local population with the weight of "cultural fitness" of the two dialects. The cultural fitness is the sum of "attractiveness" of the dialect and an additional term that increases with the fraction of the speakers of the dialect, in which the latter indicates conformity of the people. Analysis of the dynamics reveal that the evolutionary outcome critically depends on how conformity works: conformity enhances the effect of differential attractiveness between the local dialect and the standard language if conformity favors only those states in which all speakers use the same dialect (unanimity pressure model); but conformity has no effect at all if it works in proportion to the fraction among peers (peer pressure model).

\subsection{Methods}

We created a model to address the competition dynamics between a standard language $(A)$ and a local dialect $(B)$. We assume each individual chooses which dialect $(A$ or $B$ ) to use in two different situations: public and private. For the sake of clarity, we will use capital letters $(A$ and $B)$ for $A$ 
and $B$ dialects when used in public situations and small letters ( $a$ and $b)$ for when they are used in private situations.

First, we analyzed the dynamics of the public and private languages, separately. We consider three factors influence the dynamics of the public and private situations: the attractiveness of $A$ and $B$ ( $\pi_{A}$ and $\pi_{B}$, and $\pi_{a}$ and $\pi_{b}$, for the public and private languages, respectively) and the conformity factor $(\xi)$. The preference for a specific language may come from several reasons. In particular, we believe the preference for the public language will be influenced by economical aspects, while the preference for the private language is being affected by the local pride of speaking one language.

The main difference between the public and private language dynamics is that, in the private language dynamics, we considered that individuals are divided in smaller groups of size $n$. This, in turn, affects the way conformity plays a role in the decision of individuals. We assume that while in the public language dynamics, individuals are willing to conform to a language according to the fraction of speakers of that language in the population, in the private language dynamics, individuals will try to conform according to the fraction of speakers inside the group.

We investigated the effects of conformity in the private language dynamics. We consider individuals may change their language due to conformity by observing the others: the more individuals speaking other language, the larger the effect. Consequently, the dialect with more speakers tends to be favored, disregard of how attractive it is compared to the other dialect.

\subsubsection{Public language dynamics}

In the public language dynamics, each speaker changes her dialect at random times, with a rate that is determined by the attractiveness of the target dialect and the conformity factor. The conformity factor indicates that the speed of this change increases with the fraction of speakers of the target dialect in the population. Thus, the rate at which a speaker of dialect A changes to a speaker of dialect $\mathrm{B}$ is $\pi_{B}+\xi z_{B}$, where $\pi_{B}$ is the attractiveness of dialect $B, \xi$ is the conformity factor, and $z_{B}$ is the fraction of speakers of dialect $B$ in the population, with $z_{B}=1-z_{A}$ fig. 5.1. 


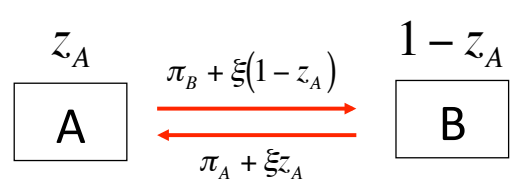

Figure 5.1: Schematic figure of public language dynamics. Individuals choose their languages according to their preferences and are also influenced by the number of individuals who speak each language. $\pi_{A}$ is preference regarding speaking $\mathrm{A}, \pi_{B}$ for preference regarding speaking $\mathrm{B}, \xi$ is the conformity factor

Analogously, the rate at which a speaker of dialect $B$ becomes a dialect $A$ speaker is $\pi_{A}+\xi z_{A}$. Thus, we can describe the dynamics of $z_{A}$ by a single equation:

$$
\begin{aligned}
\frac{d z_{A}}{d t} & =-\left(\pi_{B}+\xi\left(1-z_{A}\right)\right) z_{A}+\left(\pi_{A}+\xi z_{A}\right)\left(1-z_{A}\right) \\
& =\pi_{A}-z_{A}\left(\pi_{A}+\pi_{B}\right)
\end{aligned}
$$

Calculating the equilibrium fraction, $z_{A}^{*}$, is straightforward from eq. (5.1) and we can see that $z_{A}^{*}=\frac{\pi_{A}}{\pi_{A}+\pi_{B}}$. Furthermore, in the public language dynamics eq. (5.1), not only the fraction of $A$ speakers, $z_{A}$, is independent of the conformity factor, $\xi$, but the convergence speed is also independent of $\xi$.

\subsubsection{Private language dynamics with peer pressure model of conformity}

In the private language dynamics, individuals form groups within which they interact strongly. For instance, these groups may be composed of family members or close friends. For the sake of simplicity, we here assume that all groups have the same size, $n$. If groups are pairs $(n=2)$, they are classified in three different types: pairs in which both individuals speak dialect $A(a a)$, pairs in which both individuals speak dialect $B(b b)$, and pairs that include one dialect $A$ speaker and one dialect $B$ speaker $(a b)$.

In general, if each group is composed of $n$ individuals, then, we have $n+1$ group types in the population. Let $z_{m}$, with $m \in\{0,1, \ldots n\}$, be the fraction of groups with exactly $m A$ speakers and, consequently, $(n-m) B$ speakers. Also, we would like to define $z_{A}$ to be the fraction of $A$ speakers over all groups with different compositions. Thus, $z_{A}$ is calculated as the weighted sum of the fractions of the different group types: $z_{A}=\sum_{m=1}^{n} \frac{m z_{m}}{n}$.

We assume that individuals observe their peers to make a decision about which dialect to use. In this sense, this model of introducing the conformity factor can be called peer pressure model. We 
first analyze the cases $n=2$ and $n=3$, when groups are composed of pairs of individuals (three types of pairs: $b b, a b, a a$ ) or by triplets of individuals (four types of triplets: $b b b, b b a, b a a, a a a$ ), respectively.

\section{Pairs}

Figure 5.2 shows how the peer pressure model works when $n=2$.

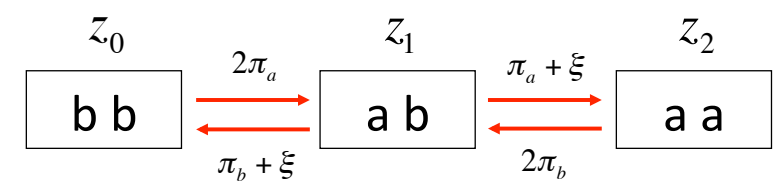

Figure 5.2: Schematic figure of peer pressure model for $n=2$. Individuals are divided in groups of 2 individuals, who can be labeled $a$ or $b$, according to the language they use in private. In the peer pressure model, the pressure to conform to one language is higher as the number of speakers of that language increases inside the group. $\pi_{a}$ is preference regarding speaking $\mathrm{A}$ in private; $\pi_{b}$ is the preference regarding speaking B in private; $\xi$ is the conformity factor.

These dynamics between the groups with pairs are described by the following equations:

$$
\begin{aligned}
& \frac{d z_{0}}{d t}=-2 \pi_{a} z_{0}+\left(\pi_{b}+\xi\right) z_{1} \\
& \frac{d z_{1}}{d t}=-\left(\pi_{a}+\pi_{b}+2 \xi\right) z_{1}+2 \pi_{b} z_{0}+2 \pi_{a} z_{2} \\
& \frac{d z_{2}}{d t}=-2 \pi_{b} z_{2}+\left(\pi_{a}+\xi\right) z_{1} .
\end{aligned}
$$

Since $z_{0}+z_{1}+z_{2}=1$, we can calculate the equilibrium fractions of the pairs in the population:

$$
\begin{aligned}
& z_{0}^{*}=\frac{\pi_{b}\left(\pi_{b}+\xi\right)}{\left(\pi_{a}+\pi_{b}\right)\left(\pi_{a}+\pi_{b}+\xi\right)} \\
& z_{1}^{*}=\frac{2 \pi_{a} \pi_{b}}{\left(\pi_{a}+\pi_{b}\right)\left(\pi_{a}+\pi_{b}+\xi\right)} \\
& z_{2}^{*}=\frac{\pi_{a}\left(\pi_{a}+\xi\right)}{\left(\pi_{a}+\pi_{b}\right)\left(\pi_{a}+\pi_{b}+\xi\right)} .
\end{aligned}
$$

From this, we see that the final fraction of the heterogeneous pair $(a b)$ decreases with $\xi$ in the

population. However, by calculating $z_{A}^{*}=\frac{z_{1}^{*}+2 z_{2}^{*}}{2}$, we observe that there is no effect of $\xi$ in the equilibrium fraction of $A$ speakers in the population: $z_{A}^{*}=\frac{\pi_{a}}{\pi_{a}+\pi_{b}}$.

\section{Triplets}

The set of equations for $n=3$ is analogous (see fig. 5.3) and we can conduct the same analysis that was done for $n=2$. 


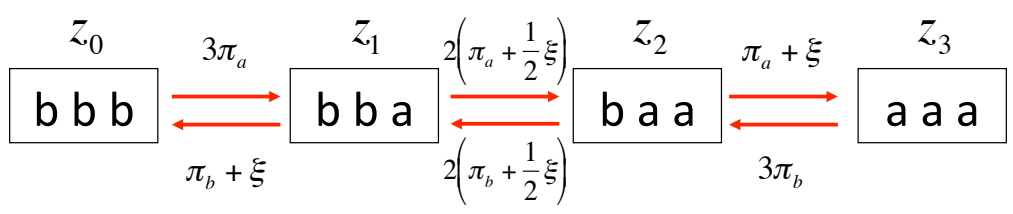

Figure 5.3: Schematic figure of peer pressure model for $n=3$. Individuals are divided in groups of 3 individuals, who can be labeled $a$ or $b$, according to the language they use in private. In the peer pressure model, the pressure to conform to one language is higher as the number of speakers of that language increases inside the group. $\pi_{a}$ is preference regarding speaking $\mathrm{A}$ in private; $\pi_{b}$ is the preference regarding speaking B in private; $\xi$ is the conformity factor.

The dynamics of the fractions of the triplets are given by:

$$
\begin{aligned}
\frac{d z_{0}}{d t} & =-3 \pi_{a} z_{0}+\left(\pi_{b}+\xi\right) z_{1} \\
\frac{d z_{1}}{d t} & =-\left(2 \pi_{a}+\pi_{b}+2 \xi\right) z_{1}+3 \pi_{a} z_{0}+\left(2 \pi_{b}+\xi\right) z_{2} \\
\frac{d z_{2}}{d t} & =-\left(\pi_{a}+2 \pi_{b}+2 \xi\right) z_{2}+\left(2 \pi_{a}+\xi\right) z_{1}+3 \pi_{b} z_{3} \\
\frac{d z_{3}}{d t} & =-3 \pi_{b} z_{3}+\left(\pi_{a}+\xi\right) z_{2},
\end{aligned}
$$

with $\sum_{m=0}^{n} z_{m}=1$.

From this system of differential equations (eq. (5.4)), we can calculate the equilibrium fractions $z_{0}^{*}$, $z_{1}^{*}, z_{2}^{*}$, and $z_{3}^{*}$ :

$$
\begin{aligned}
& z_{0}^{*}=\frac{\pi_{b}\left(\pi_{b}+\xi\right)\left(2 \pi_{b}+\xi\right)}{\left(\pi_{a}+\pi_{b}\right)\left[2\left(\pi_{a}+\pi_{b}\right)^{2}+3 \xi\left(\pi_{a}+\pi_{b}\right)+\xi^{2}\right]} \\
& z_{1}^{*}=\frac{3 \pi_{a} \pi_{b}\left(2 \pi_{b}+\xi\right)}{\left(\pi_{a}+\pi_{b}\right)\left[2\left(\pi_{a}+\pi_{b}\right)^{2}+3 \xi\left(\pi_{a}+\pi_{b}\right)+\xi^{2}\right]} \\
& z_{2}^{*}=\frac{3 \pi_{a} \pi_{b}\left(2 \pi_{a}+\xi\right)}{\left(\pi_{a}+\pi_{b}\right)\left[2\left(\pi_{a}+\pi_{b}\right)^{2}+3 \xi\left(\pi_{a}+\pi_{b}\right)+\xi^{2}\right]} \\
& z_{3}^{*}=\frac{\pi_{a}\left(\pi_{a}+\xi\right)\left(2 \pi_{a}+\xi\right)}{\left(\pi_{a}+\pi_{b}\right)\left[2\left(\pi_{a}+\pi_{b}\right)^{2}+3 \xi\left(\pi_{a}+\pi_{b}\right)+\xi^{2}\right]} .
\end{aligned}
$$

We can see from eq. (5.5) that the final fractions of all group types have some dependence on the conformity level $\xi$. In fact, as observed for $n=2$, a larger $\xi$ changes the distribution of group types such that the fractions of homogeneous groups ( $a a a$ and $b b b)$ increase, while the fractions of heterogeneous groups ( $a b b$ and $a a b)$ decrease. However, the equilibrium fraction $z_{A}^{*}$ of A speakers in the population depends only on the attractiveness levels of the standard language $A$ and the 
local dialect $B$ in private, $\pi_{a}$ and $\pi_{b}$ :

$$
\begin{aligned}
z_{A}^{*} & =\frac{z_{1}^{*}+2 z_{2}^{*}+3 z_{3}^{*}}{3} \\
& =\frac{3 \pi_{a} \pi_{b}\left(2 \pi_{b}+\xi\right)+6 \pi_{a} \pi_{b}\left(2 \pi_{a}+\xi\right)+3 \pi_{a}\left(\pi_{a}+\xi\right)\left(2 \pi_{a}+\xi\right)}{3\left(\pi_{a}+\pi_{b}\right)\left[2\left(\pi_{a}+\pi_{b}\right)^{2}+3 \xi\left(\pi_{a}+\pi_{b}\right)+\xi^{2}\right]} \\
& =\frac{\pi_{a}\left[2\left(\pi_{a}+\pi_{b}\right)^{2}+3 \xi\left(\pi_{a}+\pi_{b}\right)+\xi^{2}\right]}{\left(\pi_{a}+\pi_{b}\right)\left[2\left(\pi_{a}+\pi_{b}\right)^{2}+3 \xi\left(\pi_{a}+\pi_{b}\right)+\xi^{2}\right]} \\
& =\frac{\pi_{a}}{\pi_{a}+\pi_{b}} .
\end{aligned}
$$

Thus,the equilibrium fractions of standard language or local dialect speakers are independent of the conformity factor, $\xi$.

\section{General group size}

This independence of $\xi$ on the fraction $z_{A}$ is also observed for other values of $n$. One simple way to see this result holds is to observe that, in equilibrium, the mean transition frequency from $A$ to $B$ is the same as the mean transition frequency from $B$ to $A$. The mean conformity effect depends on which group type an individual is part of. Suppose an individual that speaks $A$ is in the group type $m$, with $m A$ speakers. This means that the conformity effect for this individual to change from $A$ to $B$ is given by $\xi(n-m) /(n-1)$. By contrast, the conformity effect for an individual that speaks $B$ to become an $A$ speaker in this same group would be given by $\xi m /(n-1)$.

More formally, we can generalize the differential equations for any $n \geq 2$, as shown below:

$$
\begin{aligned}
\frac{d z_{0}}{d t} & =-n \pi_{a} z_{0}+\left(\pi_{b}+\xi\right) z_{1} \\
\frac{d z_{j}}{d t} & =-\left[(n-j) \pi_{a}+j \pi_{b}+\frac{2 j(n-j) \xi}{n-1}\right] z_{j} \\
& +(n-j+1)\left(\pi_{a}+\frac{(j-1) \xi}{n-1}\right) z_{j-1} \\
& +(j+1)\left(\pi_{b}+\frac{(n-j-1) \xi}{n-1}\right) z_{j+1} \\
\frac{d z_{n}}{d t} & =-n \pi_{b} z_{n}+\left(\pi_{a}+\xi\right) z_{n-1}
\end{aligned}
$$

with $j=1,2, \ldots, n-1$. 
Thus, we can calculate $d z_{A} / d t$ by using the definition of $z_{A}$, given by $z_{A}=\frac{1}{n} \sum_{j=1}^{n} j z_{j}$ :

$$
\frac{d z_{A}}{d t}=\frac{d}{d t}\left(\frac{1}{n} \sum_{j=1}^{n} j z_{j}\right)=\frac{1}{n} \sum_{j=1}^{n} j \frac{d z_{j}}{d t}
$$

Replacing the terms from eqs. (5.7b) and (5.7c), we get the following equation:

$$
\begin{aligned}
\frac{d z_{A}}{d t} & =\frac{-1}{n} \sum_{j=1}^{n-1} j\left[(n-j) \pi_{a}+j \pi_{b}+\frac{2(n-j) j \xi}{n-1}\right] z_{j} \\
& +\frac{1}{n} \sum_{j=1}^{n-1} j(n-j+1)\left(\pi_{a}+\frac{(j-1) \xi}{n-1}\right) z_{j-1} \\
& +\frac{1}{n} \sum_{j=1}^{n-1} j(j+1)\left(\pi_{b}+\frac{(n-j-1) \xi}{n-1}\right) z_{j+1} \\
& -n \pi_{b} z_{n}+\left(\pi_{a}+\xi\right) z_{n-1}
\end{aligned}
$$

We can make a simple variable change in the last two summation terms so that they are also written in function of $z_{j}$. This change yields:

$$
\begin{aligned}
\frac{d z_{A}}{d t} & =\frac{-1}{n} \sum_{j=1}^{n-1} j\left[(n-j) \pi_{a}+j \pi_{b}+\frac{2(n-j) j \xi}{n-1}\right] z_{j} \\
& +\frac{1}{n} \sum_{j=0}^{n-2}(j+1)(n-j)\left(\pi_{a}+\frac{j \xi}{n-1}\right) z_{j} \\
& +\frac{1}{n} \sum_{j=2}^{n}(j-1) j\left(\pi_{b}+\frac{(n-j) \xi}{n-1}\right) z_{j} \\
& -n \pi_{b} z_{n}+\left(\pi_{a}+\xi\right) z_{n-1}
\end{aligned}
$$

Thus, we have some expression which can be written as:

$$
\frac{d z_{A}}{d t}=\sum_{j=2}^{n-2} a_{j} z_{j}+C
$$

The coefficients $a_{j}$ for $z_{j}$, for $j=2,3, \ldots, n-2$ can be calculated from eq. (5.10):

$$
\begin{aligned}
n a_{j} & =-j(n-j) \pi_{a}-j^{2} \pi_{b}-\frac{2(n-j) j^{2} \xi}{n-1}+(n-j)(j+1) \pi_{a} \\
& +\frac{(n-j) j(j+1) \xi}{n-1}+(j-1) j \pi_{b}+\frac{(n-j) j(j-1) \xi}{n-1}
\end{aligned}
$$


Hence, after some algebraic manipulations, we can find that the expression for $a_{j}$ is given by:

$$
a_{j}=\frac{1}{n}\left[(n-j) \pi_{a}-j \pi_{b}\right]
$$

The independent term $C$ is formed from the terms outside the summation term (for $j=2,3, \ldots, n-$ 2), cf. eq. (5.11), and can be written as:

$$
\begin{aligned}
n C & =-\left[(n-1) \pi_{a}+\pi_{b}+2 \xi\right] z_{1}-(n-1)\left[\pi_{a}+(n-1) \pi_{b}+2 \xi\right] z_{n-1} \\
& +n \pi_{a} z_{0}+2\left[(n-1) \pi_{a}+\xi\right] z_{1}+(n-2)(n-1)\left(\pi_{b}+\frac{\xi}{n-1}\right) z_{n-1} \\
& +(n-1) n \pi_{b} z_{n}-n^{2} \pi_{b} z_{n}+n\left(\pi_{a}+\xi\right) z_{n-1}
\end{aligned}
$$

Since $z_{0}=1-\sum_{j=1}^{n} z_{j}$, we can group the terms for each $z_{i}$, getting the following expression for $C$ :

$$
C=\pi_{a}-\left(\pi_{a}+\pi_{b}\right)\left[z_{1}+(n-1) z_{n-1}+n z_{n}\right]-\pi_{a} \sum_{j=2}^{n-2} z_{j}
$$

By replacing eqs. (5.13) and (5.15) into eq. (5.11), we get:

$$
\frac{d z_{A}}{d t}=-\sum_{j=1}^{n} \frac{j\left(\pi_{a}+\pi_{b}\right) z_{j}}{n}+\pi_{a}
$$

Finally, using the definition for $z_{A}$, the final expression for $d z_{A} / d t$ can be written as:

$$
\frac{d z_{A}}{d t}=-\left(\pi_{a}+\pi_{b}\right) z_{A}+\pi_{a}
$$

From eq. (5.17), we can conclude that for any $n \geq 2$, there is no dependence on $\xi$ in both the equilibrium point and the dynamics of $z_{A}$, the average fraction of $A$ speakers in a population divided in groups of $n$ individuals. 


\subsubsection{Private language dynamics with an alternative model of conformity - the unanimity pressure model}

In the former model, we assumed that individuals would change their dialect due to conformity pressure if there is at least one individual speaking another dialect in the group. Human psychology, however, seems to work in another way. One famous study by Asch [8] reported that individuals tend to conform to the others more frequently if nobody else agrees with them.

Following Asch's experimental result, in this alternative model, conformity influences only the transitions to the extreme states (e.g. $a a b$ to $a a a$, or $a b b$ to $b b b$ ), and we call it the unanimity pressure model, described in fig. 5.4 for $n=3$. When the group size is two, both approaches yield the same set of equations, thus, here we treat the case in which $n=3$.

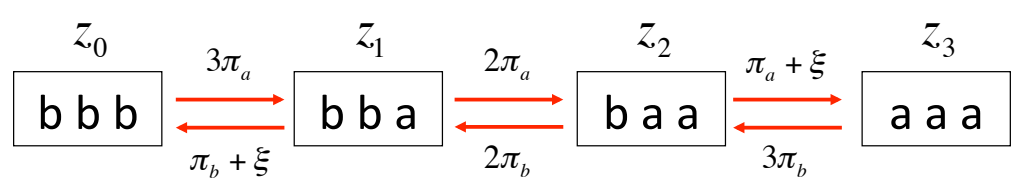

Figure 5.4: Schematic figure of unanimity pressure model. Individuals are divided in groups of 3 individuals, who can be labeled $a$ or $b$, according to the language they use in private. In the unanimity pressure model, there is pressure to conform to one language only if all the other individuals speak that language inside the group. $\pi_{a}$ is preference regarding speaking $\mathrm{A}$ in private; $\pi_{b}$ is the preference regarding speaking B in private; $\xi$ is the conformity factor.

In the unanimity pressure model, the dynamics of the fractions of the triplets are given by:

$$
\begin{aligned}
\frac{d z_{0}}{d t} & =-3 \pi_{a} z_{0}+\left(\pi_{b}+\xi\right) z_{1} \\
\frac{d z_{1}}{d t} & =-\left(2 \pi_{a}+\pi_{b}+\xi\right) z_{1}+3 \pi_{a} z_{0}+2 \pi_{b} z_{2} \\
\frac{d z_{2}}{d t} & =-\left(\pi_{a}+2 \pi_{b}+\xi\right) z_{2}+2 \pi_{a} z_{1}+3 \pi_{b} z_{3} \\
\frac{d z_{3}}{d t} & =-3 \pi_{b} z_{3}+\left(\pi_{a}+\xi\right) z_{2} .
\end{aligned}
$$

Using the assumption that $z_{0}+z_{1}+z_{2}+z_{3}=1$, we can find the equilibrium points, which are given by:

$$
\begin{aligned}
& z_{0}^{*}=\frac{\pi_{b}^{2}\left(\pi_{b}+\xi\right)}{\left(\pi_{a}+\pi_{b}\right)^{3}+\xi\left(\pi_{a}^{2}+\pi_{b}^{2}\right)} \\
& z_{1}^{*}=\frac{3 \pi_{a} \pi_{b}^{2}}{\left(\pi_{a}+\pi_{b}\right)^{3}+\xi\left(\pi_{a}^{2}+\pi_{b}^{2}\right)} \\
& z_{2}^{*}=\frac{3 \pi_{a}^{2} \pi_{b}}{\left(\pi_{a}+\pi_{b}\right)^{3}+\xi\left(\pi_{a}^{2}+\pi_{b}^{2}\right)} \\
& z_{3}^{*}=\frac{\pi_{a}^{2}\left(\pi_{a}+\xi\right)}{\left(\pi_{a}+\pi_{b}\right)^{3}+\xi\left(\pi_{a}^{2}+\pi_{b}^{2}\right)} .
\end{aligned}
$$


Hence, since $z_{A}=\left(z_{1}+2 z_{2}+3 z_{3}\right) / 3$, the equilibrium point of the fraction of A speakers in the population, $z_{A}^{*}$ is given by:

$$
z_{A}^{*}=\frac{\pi_{a}\left[\left(\pi_{a}+\pi_{b}\right)^{2}+\pi_{a} \xi\right]}{\left(\pi_{a}+\pi_{b}\right)^{3}+\xi\left(\pi_{a}^{2}+\pi_{b}^{2}\right)} .
$$

From the derivative of $z_{A}^{*}$ in relation to $\xi$ (eq. (5.21)), we can see that $\left(\pi_{a}-\pi_{b}\right)$ determines if an increase in $\xi$ will increase or decrease the fraction $z_{A}$ :

$$
\frac{\partial z_{A}^{*}}{\partial \xi}=\frac{\pi_{a} \pi_{b}\left(\pi_{a}-\pi_{b}\right)\left(\pi_{a}+\pi_{b}\right)^{2}}{\left[\left(\pi_{a}+\pi_{b}\right)^{3}+\xi\left(\pi_{a}^{2}+\pi_{b}^{2}\right)\right]^{2}} .
$$

Thus, a stronger conformity enhances the fraction of the most attractive language.

\subsection{Combining the public and private language dynamics}

Combining the public and private language dynamics can be done in several ways. Here, we use a strong assumption to make the analysis feasible: we assume that the cost of using two different dialects is infinitely large. This implies that no individual would be willing to speak one dialect in public while speaking another dialect in private, so that there are no mixed individuals $(A a, A b, B a$ or $B b)$ in the population. Thus, as in the private language dynamics, we have four types of groups in the population, $B B B$ (composed of three individuals $B b$ ), $B B A$ (composed of two individuals $B b$ and one individual $A a), B A A, A A A$.

We are also assuming that there is a parameter $\lambda_{\text {pub }}$ which determines how much the public interactions influence on the choice of the dialect. Analogously, $\lambda_{\text {priv }}$ measures the influence of private interactions on the choice of a dialect, and $\lambda_{\text {priv }}=1-\lambda_{\text {pub }}$, with $\lambda_{\text {pub }} \in[0,1]$. When $\lambda_{\text {pub }}=1$, private interactions are not taken into account and we have a model that represents an only public language dynamics. Conversely, if $\lambda_{\text {priv }}=1$, we have a model that corresponds exactly to the private language dynamics, studied in the previous section.

\subsubsection{Combined language dynamics with peer pressure model of conformity}

Again, let $z_{i}$ be the fraction of the group types that have $i A$ speakers in the group. In fig. 5.5, we can see a scheme of how are the transition rates between different $z_{i}$, for $i=0,1,2,3$. 
This implies that, for $n=3$, we have the following set of equations:

$$
\begin{aligned}
\frac{d z_{0}}{d t} & =-3\left[\lambda_{\text {pub }}\left(\pi_{A}+\xi z_{A}\right)+\lambda_{\text {priv }} \pi_{a}\right] z_{0}+\left[\lambda_{\text {pub }}\left(\pi_{B}+\xi\left(1-z_{A}\right)\right)+\lambda_{\text {priv }}\left(\pi_{b}+\xi\right)\right] z_{1} \\
\frac{d z_{1}}{d t} & =-\left[\lambda_{\text {pub }}\left(2 \pi_{A}+\pi_{B}+\xi\left(1+z_{A}\right)\right)+\lambda_{\text {priv }}\left(2 \pi_{a}+\pi_{b}+2 \xi\right)\right] z_{1} \\
& +3\left[\lambda_{\text {pub }}\left(\pi_{A}+\xi z_{A}\right)+\lambda_{\text {priv }} \pi_{a}\right] z_{0}+2\left[\lambda_{\text {pub }}\left(\pi_{B}+\xi\left(1-z_{A}\right)\right)+\lambda_{\text {priv }}\left(\pi_{b}+\frac{1}{2} \xi\right)\right] z_{2} \\
\frac{d z_{2}}{d t} & =-\left[\lambda_{\text {pub }}\left(\pi_{A}+2 \pi_{B}+\xi\left(2-z_{A}\right)\right)+\lambda_{\text {priv }}\left(\pi_{a}+2 \pi_{b}+2 \xi\right)\right] z_{2} \\
& +2\left[\lambda_{\text {pub }}\left(\pi_{A}+\xi z_{A}\right)+\lambda_{\text {priv }}\left(\pi_{a}+\frac{1}{2} \xi\right)\right] z_{1}+3\left[\lambda_{\text {pub }}\left(\pi_{B}+\xi\left(1-z_{A}\right)\right)+\lambda_{\text {priv }} \pi_{b}\right] z_{3} \\
\frac{d z_{3}}{d t} & =-3\left[\lambda_{\text {pub }}\left(\pi_{B}+\xi\left(1-z_{A}\right)\right)+\lambda_{\text {priv }} \pi_{b}\right] z_{3}+\left[\lambda_{\text {pub }}\left(\pi_{A}+\xi z_{A}\right)+\lambda_{\text {priv }}\left(\pi_{a}+\xi\right)\right] z_{2},
\end{aligned}
$$

with $\sum_{m=0}^{n} z_{m}=1$.

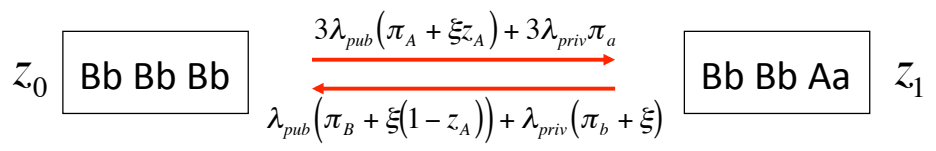

$$
\begin{aligned}
& \begin{array}{l}
2 \lambda_{\text {pub }}\left(\pi_{B}+\xi\left(1-z_{A}\right)\right) \\
+2 \lambda_{\text {priv }}\left(\pi_{b}+\frac{1}{2} \xi\right)
\end{array} \mid \uparrow \begin{array}{l}
2 \lambda_{\text {pub }}\left(\pi_{A}+\xi z_{A}\right) \\
+2 \lambda_{\text {priv }}\left(\pi_{a}+\frac{1}{2} \xi\right)
\end{array} \\
& z_{3} \text { Aa Aa Aa } \underset{\lambda_{\text {pub }}\left(\pi_{A}+\xi z_{A}\right)+\lambda_{\text {priv }}\left(\pi_{a}+\xi\right)}{\stackrel{3 \lambda_{\text {pub }}\left(\pi_{B}+\xi\left(1-z_{A}\right)\right)+3 \lambda_{\text {priv }} \pi_{b}}{\rightleftarrows}} \text { Bb Aa Aa } z_{2}
\end{aligned}
$$

Figure 5.5: Schematic figure of the combined dynamics for the peer pressure model. Individuals choose their languages influenced by preferences in both public and private situations and receive pressure to conform in private according to the peer pressure model. $\lambda_{\text {pub }}\left(\lambda_{\text {priv }}\right)$ is the weight of public (private) interactions; $\pi_{A}\left(\pi_{a}\right)$ is preference regarding speaking $\mathrm{A}$ in public (in private); $\pi_{B}$ $\left(\pi_{b}\right)$ is the preference regarding speaking B in public (in private); $\xi$ is the conformity factor.

By using the previous results of the public situation model and the private situation with the peer pressure model and considering $\lambda_{\text {priv }}=1-\lambda_{\text {pub }}$, we can calculate the equilibrium fraction of $z_{A}$ :

$$
z_{A}^{*}=\frac{\lambda_{p u b}\left(\pi_{A}-\pi_{a}\right)+\pi_{a}}{\lambda_{p u b}\left(\pi_{A}-\pi_{a}+\pi_{B}-\pi_{b}\right)+\pi_{a}+\pi_{b}} .
$$

\subsubsection{Combined language dynamics with unanimity pressure model of confor- mity}

In the unanimity pressure model, the transition rates between the states are different in the private component, as shown in fig. 5.6, depicting the case in which $n=3$. 


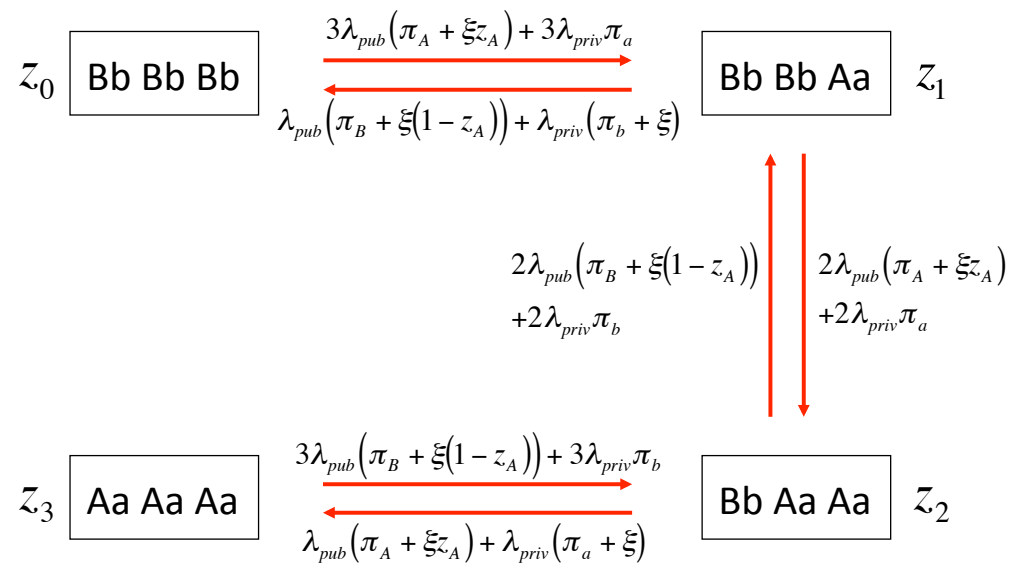

Figure 5.6: Schematic figure of the combined dynamics for the unanimity pressure model. Individuals choose their languages influenced by preferences in both public and private situations and receive pressure to conform in private according to the unanimity pressure model. $\lambda_{\text {pub }}\left(\lambda_{\text {priv }}\right)$ is the weight of public (private) interactions; $\pi_{A}\left(\pi_{a}\right)$ is preference regarding speaking A in public (in private); $\pi_{B}\left(\pi_{b}\right)$ is the preference regarding speaking B in public (in private); $\xi$ is the conformity factor.

From these transition rates, we can write the following equations:

$$
\begin{aligned}
\frac{d z_{0}}{d t} & =-3\left[\lambda_{\text {pub }}\left(\pi_{A}+\xi z_{A}\right)+\lambda_{\text {priv }} \pi_{a}\right] z_{0}+\left[\lambda_{\text {pub }}\left(\pi_{B}+\xi\left(1-z_{A}\right)\right)+\lambda_{\text {priv }}\left(\pi_{b}+\xi\right)\right] z_{1} \\
\frac{d z_{1}}{d t} & =-\left[\lambda_{\text {pub }}\left(2 \pi_{A}+\pi_{B}+\xi\left(1+z_{A}\right)\right)+\lambda_{\text {priv }}\left(2 \pi_{a}+\pi_{b}+\xi\right)\right] z_{1} \\
& +3\left[\lambda_{\text {pub }}\left(\pi_{A}+\xi z_{A}\right)+\lambda_{\text {priv }} \pi_{a}\right] z_{0}+2\left[\lambda_{\text {pub }}\left(\pi_{B}+\xi\left(1-z_{A}\right)\right)+\lambda_{\text {priv }} \pi_{b}\right] z_{2} \\
\frac{d z_{2}}{d t} & =-\left[\lambda_{\text {pub }}\left(\pi_{A}+2 \pi_{B}+\xi\left(2-z_{A}\right)\right)+\lambda_{\text {priv }}\left(\pi_{a}+2 \pi_{b}+\xi\right)\right] z_{2} \\
& +2\left[\lambda_{\text {pub }}\left(\pi_{A}+\xi z_{A}\right)+\lambda_{\text {priv }} \pi_{a}\right] z_{1}+3\left[\lambda_{\text {pub }}\left(\pi_{B}+\xi\left(1-z_{A}\right)\right)+\lambda_{\text {priv }} \pi_{b}\right] z_{3} \\
\frac{d z_{3}}{d t} & =-3\left[\lambda_{\text {pub }}\left(\pi_{B}+\xi\left(1-z_{A}\right)\right)+\lambda_{\text {priv }} \pi_{b}\right] z_{3}+\left[\lambda_{\text {pub }}\left(\pi_{A}+\xi z_{A}\right)+\lambda_{\text {priv }}\left(\pi_{a}+\xi\right)\right] z_{2},
\end{aligned}
$$

with $\sum_{m=0}^{n} z_{m}=1$.

Since it is not feasible to get analytical results for the stable fractions of $z_{i}, i=0,1,2,3$, we analyze these equations through numerical simulations as shown in the next section.

\subsection{Results}

In this section, we show the results of our numerical analyses obtained from the equations of our models. We divided this section in two parts. In the first part, we explain the results related to the peer pressure model. In the second part, we explain the results related to the unanimity pressure model. 


\subsubsection{Peer pressure model}

In fig. 5.7, we see how the distribution varies according to the conformity factor $\xi$ for the private language dynamics in the peer pressure model. We can see that in the left panel (fig. 5.7a), when $\xi=1$, the distribution is slightly right skewed, since $\pi_{b}>\pi_{a}$. However, when the conformity factor is larger, $\xi=100$, in the right panel (fig. 5.7b), we see that the intermediate heterogeneous group fractions are reduced to almost zero and the groups are distributed in groups of types $a a a$ and $b b b$. There are more groups of types $b b b$, since $\pi_{b}>\pi_{a}$, and as the difference between $\pi_{a}$ and $\pi_{b}$ gets larger, the distribution is more skewed for small $\xi$ and the difference between the levels of $z_{0}$ and $z_{3}$ becomes more pronounced.

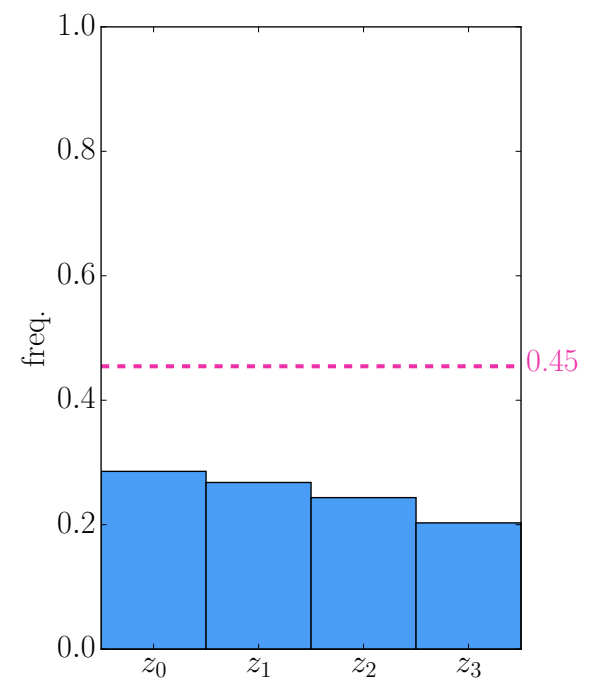

(a) $\xi=1$

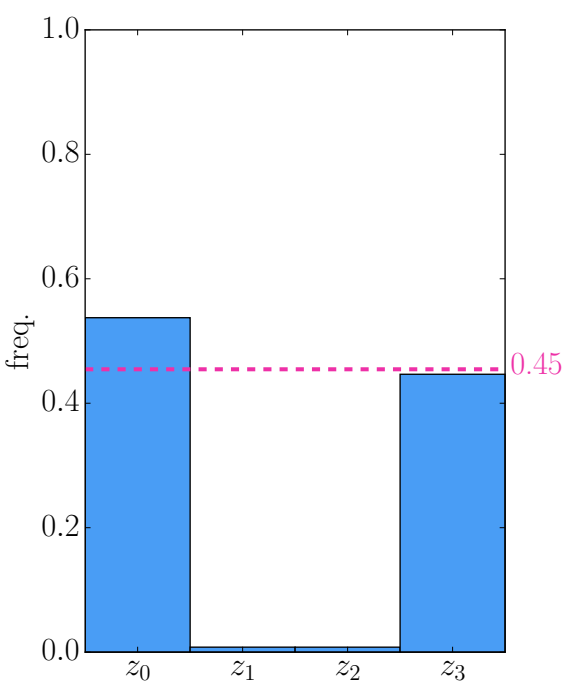

(b) $\xi=100$

Figure 5.7: Equilibrium distribution of group types fractions in equilibrium for the peer pressure model with $n=3$ for different conformity levels $(\xi)$. $z_{i}$ corresponds to the fraction of the group type with $i A$ speakers. The dashed pink line represents the equilibrium fraction of $A$ speakers in the population, $z_{A}$. Private language dynamics with parameters: $\pi_{a}=0.5, \pi_{b}=0.6$.

The pink line in both panels in fig. 5.7 is the average value, i.e., the average number of $A$ speakers in the population, $z_{A}$. This line is exactly at the same height in both panels, since the final fraction of $z_{A}$ is independent of $\xi$, as proven in eq. (5.6).

The independence on the conformity factor can also be observed in fig. 5.8. In this figure, we plotted the curves for the final fraction of $A$ speakers in function of $\pi_{a}$, the attractiveness of language $A$, for two different $\xi$ values. We can see that both curves coincide. 


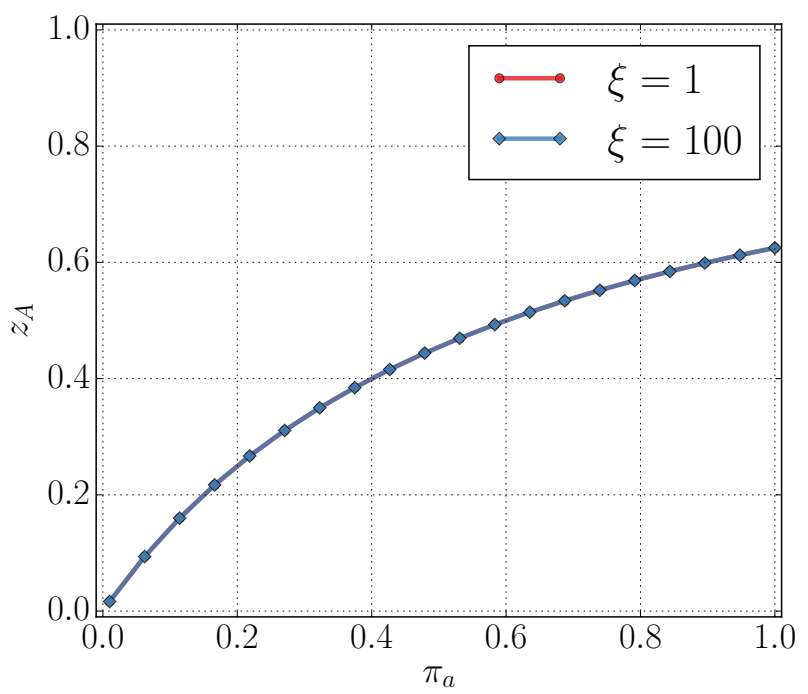

Figure 5.8: Final fraction of $A$ speakers over $\pi_{a}$ values for the peer pressure model for fixed $\pi_{b}$. The red line (with $20 \%$ of transparency) corresponds to the case in which $\xi=1$, while the blue line (with $20 \%$ of transparency) depicts the situation when $\xi=100$. Private language dynamics with fixed parameter: $\pi_{b}=0.6$.

Using the combined dynamics with the peer pressure model, we compared the trajectories of $z_{A}$ for different values of $\lambda_{p u b}$, considering a strong difference in attractiveness in the public situation $\left(\pi_{A}=0.8\right.$ and $\left.\pi_{B}=0.3\right)$ and a slight difference in attractiveness in the private situation $\left(\pi_{a}=0.5\right.$ and $\pi_{b}=0.6$ ). We can see from fig. 5.9 how larger values of $\lambda_{p u b}$ increase the final fraction of $z_{A}$. This happens for two reasons: (i) the final fraction is independent of $\xi$; and (ii) in public situations the standard language $A$ is more attractive than the local dialect $B$, i.e., $\pi_{A}>\pi_{B}$.

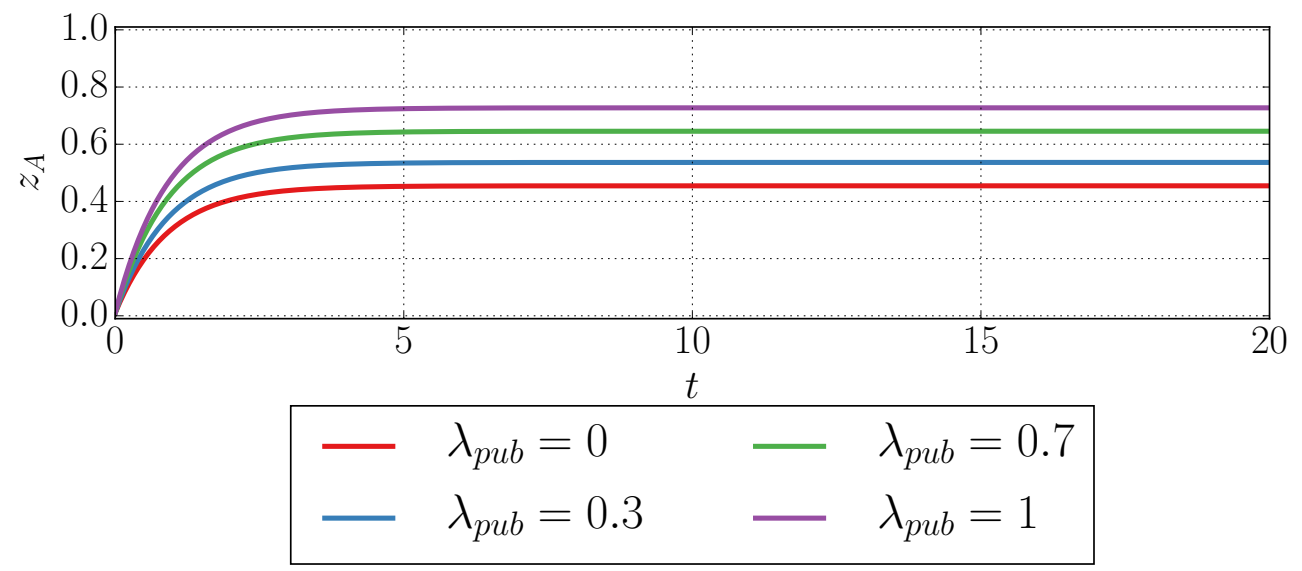

Figure 5.9: Trajectories of $z_{A}$ for different values of $\lambda_{p u b}$ using the peer pressure model. Parameters: $\pi_{A}=0.8, \pi_{B}=0.3, \pi_{a}=0.5, \pi_{b}=0.6, \xi=1$.

As previously mentioned, when $\lambda_{p u b}=0$ (red curve in fig. 5.9), the pure private language dynamics is recovered. In contrast to the results with higher levels of $\lambda_{p u b}$, in the pure private language 
dynamics, we observe that, in the equilibrium, the majority of the individuals are $B$ speakers, since public situations do not influence on the choice of the dialect and $\pi_{a}<\pi_{b}$ for private situations.

Using the formula given by 5.23 , we can calculate the critical $\lambda_{p u b}$ needed in order to have $z_{A}^{*}=0.5$. In fig. 5.10, we show how this critical $\lambda_{p u b}$ changes for different values of $\pi_{a}$ when $\pi_{b} \in\{0.3,0.5,0.7\}$. As expected, we can see that as $\pi_{a}$ increases, and $\pi_{b}$ decreases, $z_{A}>z_{B}$ is attained even for low values of $\lambda_{\text {pub }}$. By contrast, if $\pi_{b}$ is larger, then, even with $\lambda_{p u b}>0.5$, it might be possible to get the majority of individuals to speak the dialect $B$, as long as $\pi_{a}$ is sufficiently small.

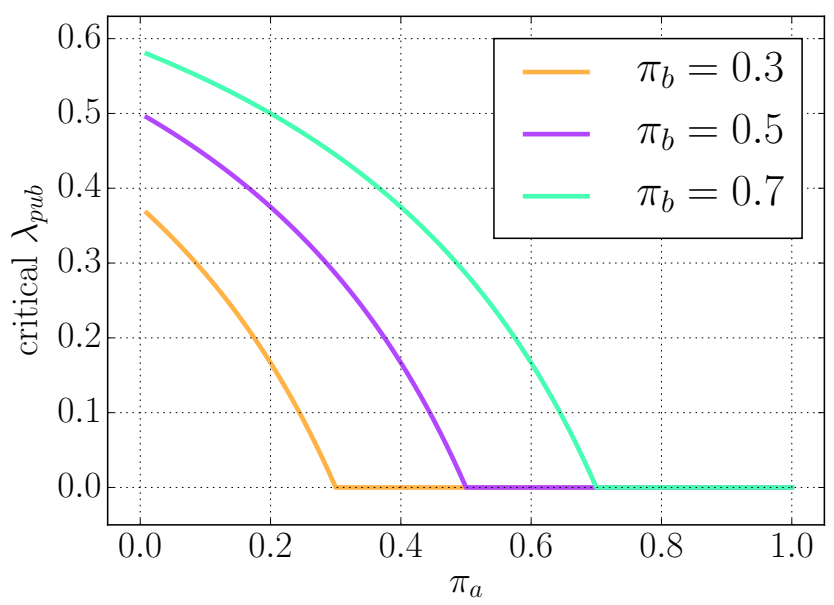

Figure 5.10: Critical values of $\lambda_{p u b}$ such that the final fraction of $z_{A}$ is larger or less than 0.5 for different levels of $\pi_{b}$ using the peer pressure model. Parameters: $\pi_{A}=0.8, \pi_{B}=0.3, \xi=1$.

\subsubsection{Unanimity pressure model}

In the unanimity pressure model, there is no conformity effects in the transition rates between heterogeneous groups and we found that $\xi$ influences both the final fractions of the group types and the final average fraction of the group types, $z_{A}$. In fig. 5.11, we compare the distribution of group types for the private language dynamics with the unanimity pressure model. The left panel (fig. 5.11a) shows the case in which $\xi=1$ and the right panel (fig. 5.11b) shows the case in which $\xi=100$. We can see that when $\xi$ is low, the distribution is skewed towards the group types with more $B$ speakers (since $\pi_{b}>\pi_{a}$ ). On the other hand, when $\xi$ is large, it makes the distribution to be concentrated mostly in the extremes, aaa $\left(z_{3}\right)$ and $b b b\left(z_{0}\right)$. A larger $\xi$ also enhances the effect dictated by $\pi_{a}$ and $\pi_{b}$, yielding a lower fraction of $z_{A}$ (pink line). 


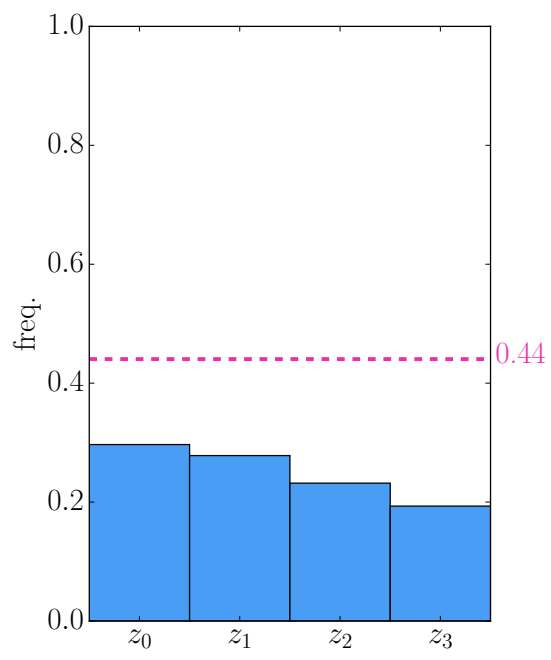

(a) $\xi=1$

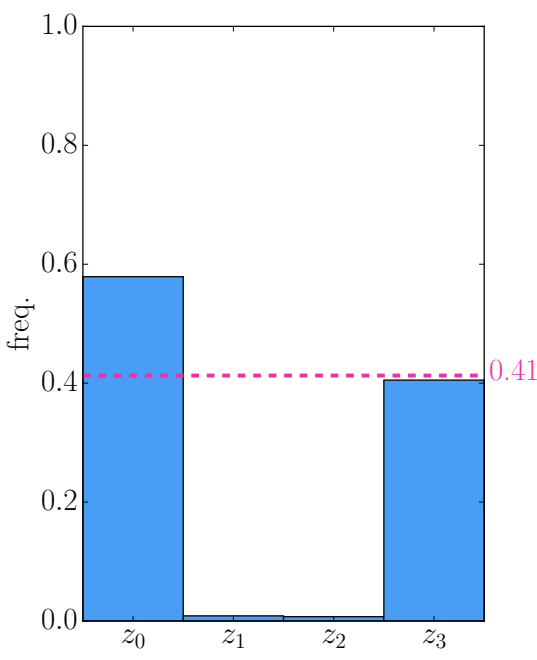

(b) $\xi=100$

Figure 5.11: Equilibrium distribution of group types fractions in equilibrium for the unanimity pressure model with $n=3$ for different conformity levels $(\xi)$. $z_{i}$ corresponds to the fraction of the group type with $i A$ speakers. The dashed pink line represents the equilibrium fraction of $A$ speakers in the population, $z_{A}$. Private language dynamics with parameters: $\pi_{a}=0.5, \pi_{b}=0.6$.

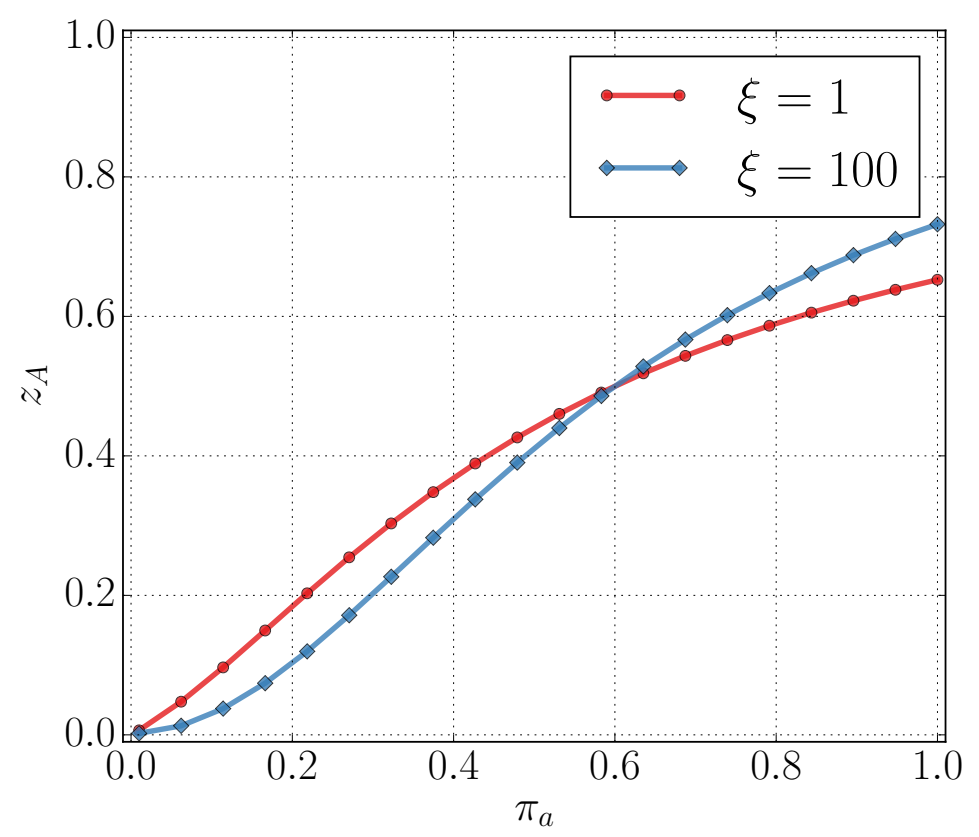

Figure 5.12: Final fraction of $A$ speakers over $\pi_{a}$ values for the unanimity pressure model for fixed $\pi_{b}$. The red line (with $20 \%$ of transparency) corresponds to the case in which $\xi=1$, while the blue line (with $20 \%$ of transparency) depicts the situation when $\xi=10$. Private language dynamics with fixed parameter: $\pi_{b}=0.6$.

We can observe more closely the effects of $\xi$ in fig. 5.12. Here, we plotted the final fraction of $A$ speakers, $z_{A}^{*}$, in function of $\pi_{a}$, for a fixed $\pi_{b}$ and two different conformity factor values. We see that 
when $\xi$ is larger, $z_{A}^{*}$ is smaller for $\pi_{a}<\pi_{b}$ and is larger for $\pi_{a}>\pi_{b}$, which shows the relationship of $z_{A}^{*}$ and $\xi$, as predicted by eq. (5.21).

Besides influencing in the equilibrium fractions of the group types and in the average equilibrium fraction of $A$ speakers, in the unanimity pressure model, different from the peer pressure model, $\xi$ affects the speed of convergence of $z_{A}$ towards the equilibrium. In fig. 5.13, we compare the private language dynamics with the unanimity pressure model for two different initial conditions at two different levels of the conformity factor $\xi$. We can see that in spite of different initial conditions, the dynamics of $z_{A}$ is led to a single equilibrium, for both low and high $\xi$ values. As seen in the previous graph, the final fraction of $z_{A}$ is dependent on $\xi$, with a larger $\xi$ leading to a lower $z_{A}^{*}$. It is also clear that the convergence towards the equilibrium is much slower for higher $\xi$ levels, and this phenomena is caused by the time taken for shifting the group distribution to the extremes, which happens when $\xi$ is larger.

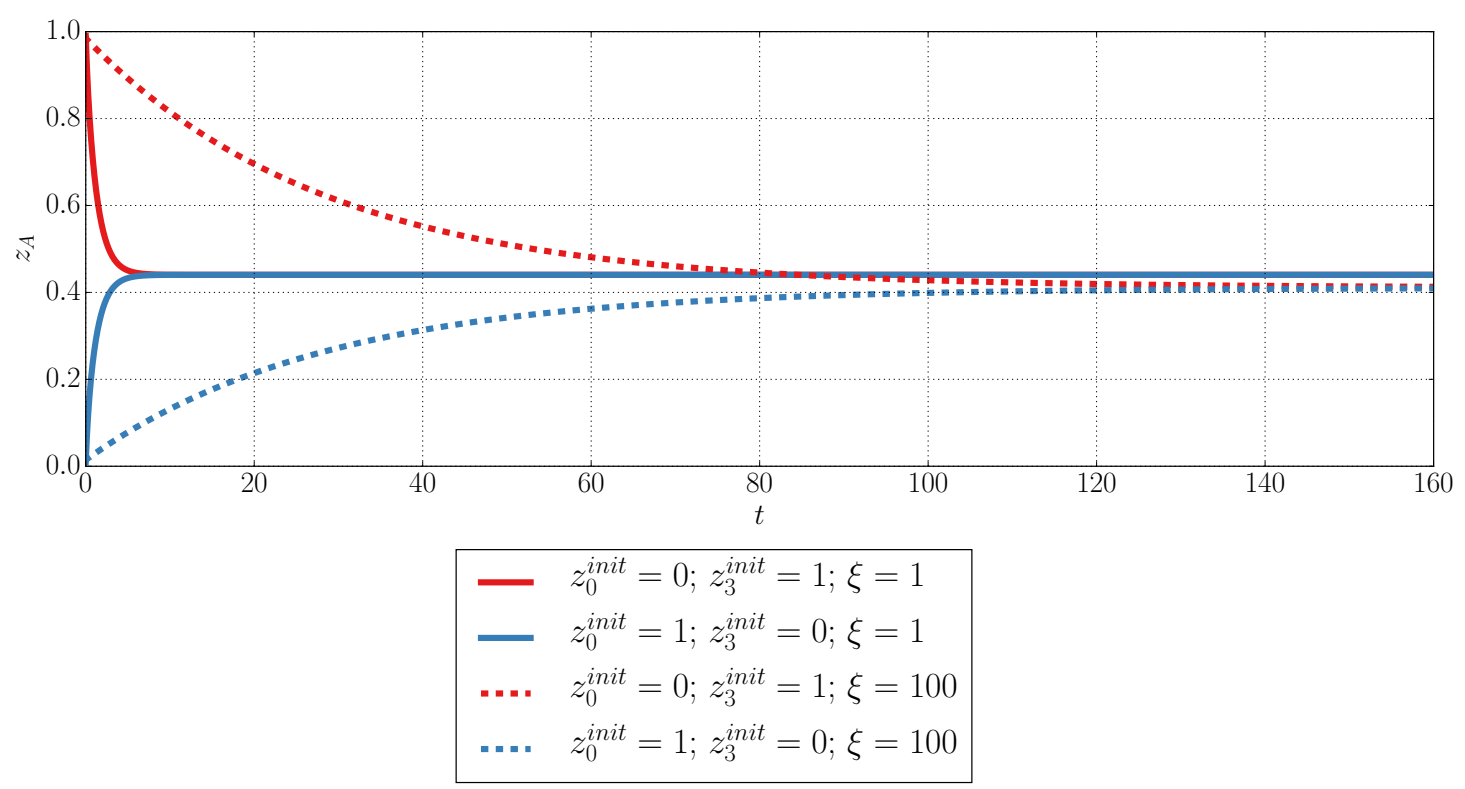

Figure 5.13: Fractions of $A$ speakers over time for different initial conditions and different $\xi$ for the unanimity pressure model. The red lines represent the trajectories when the population is initially composed only by group types with only $A$ speakers. The blue lines represent the opposite situation, in which the population is initially composed of group types with only $B$ speakers. Solid lines and dashed lines correspond to the dynamics with $\xi=1$ and $\xi=100$, respectively. Private language dynamics with parameters: $\pi_{a}=0.5, \pi_{b}=0.6$.

We can also see this difference in the convergence rates for different $\xi$ for different values of $\lambda_{\text {pub }}$ in fig. 5.14. When $\xi$ is low (top panel, fig. 5.14a), the graph is similar to the one for the peer pressure model, and as $\lambda_{p u b}$ increases, the average fraction of $A$ speakers increase as well, since $\pi_{A}>\pi_{B}$. An interesting phenomenon is observed in the bottom panel (fig. 5.14b). Larger values 
of $\xi$ allow intermediate values of $\lambda_{p u b}$ (between private and public language dynamics) to increase the final fraction $z_{A}$ up to higher levels than what is observed in the only public language dynamics $\left(\lambda_{p u b}=0\right)$.

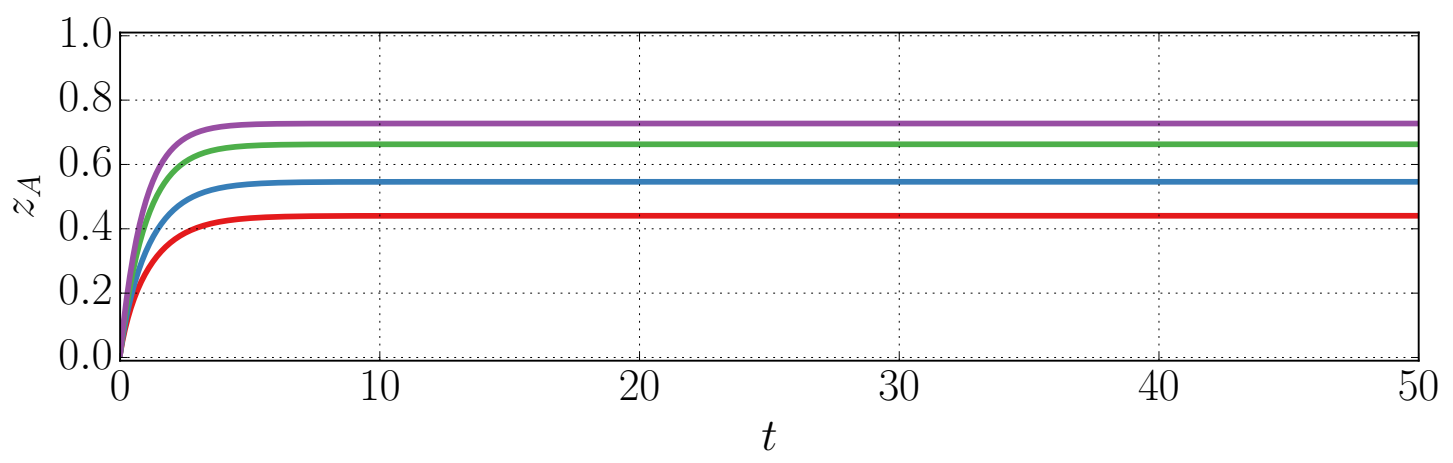

(a) $\xi=1$

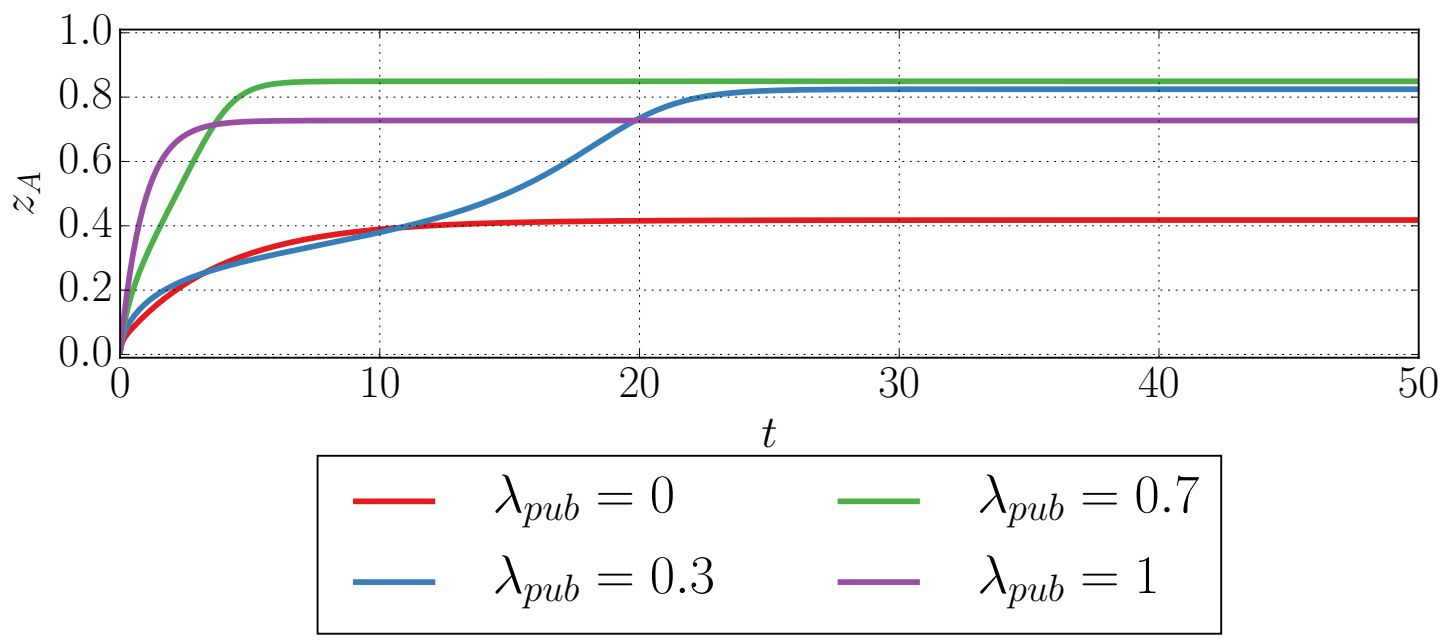

(b) $\xi=10$

Figure 5.14: Trajectories of $z_{A}$ for different values of $\lambda_{p u b}$ using the unanimity pressure model. Parameters: $\pi_{A}=0.8, \pi_{B}=0.3, \pi_{a}=0.5, \pi_{b}=0.6$

In fig. 5.15, we show how the fractions $z_{0}, z_{1}, z_{2}$ and $z_{3}$ are changing at each time step for different values of $\lambda_{p u b}$. Here, again, we are comparing two scenarios: one with $\xi=1$ (top panel, fig. 5.15a) and one with $\xi=10$ (bottom panel, 5.15b). We see that convergence time is longer when $\xi$ is large, because in this case, there is a flow from groups of type $b b b$ to $a b b$, and then, to $a a b$, and finally to aaa. Interestingly, the way the curves are ordered also changes according to $\xi$. When $\xi$ is low, $z_{0}^{*}$ and $z_{1}^{*}$ are higher the lower the value of $\lambda_{p u b}$. The opposite situation occurs for $z_{2}^{*}$ and $z_{3}^{*}$, which are higher the larger $\lambda_{p u b}$ is, reflecting the conflict of the attractiveness in public and in private. On the other hand, when $\xi=10$, there is no such pattern, and we see that for intermediate values of $\lambda_{p u b}$, the final fractions $z_{0}^{*}, z_{1}^{*}$ and $z_{2}^{*}$ are considerably lower than in the case of $\xi=1$. 

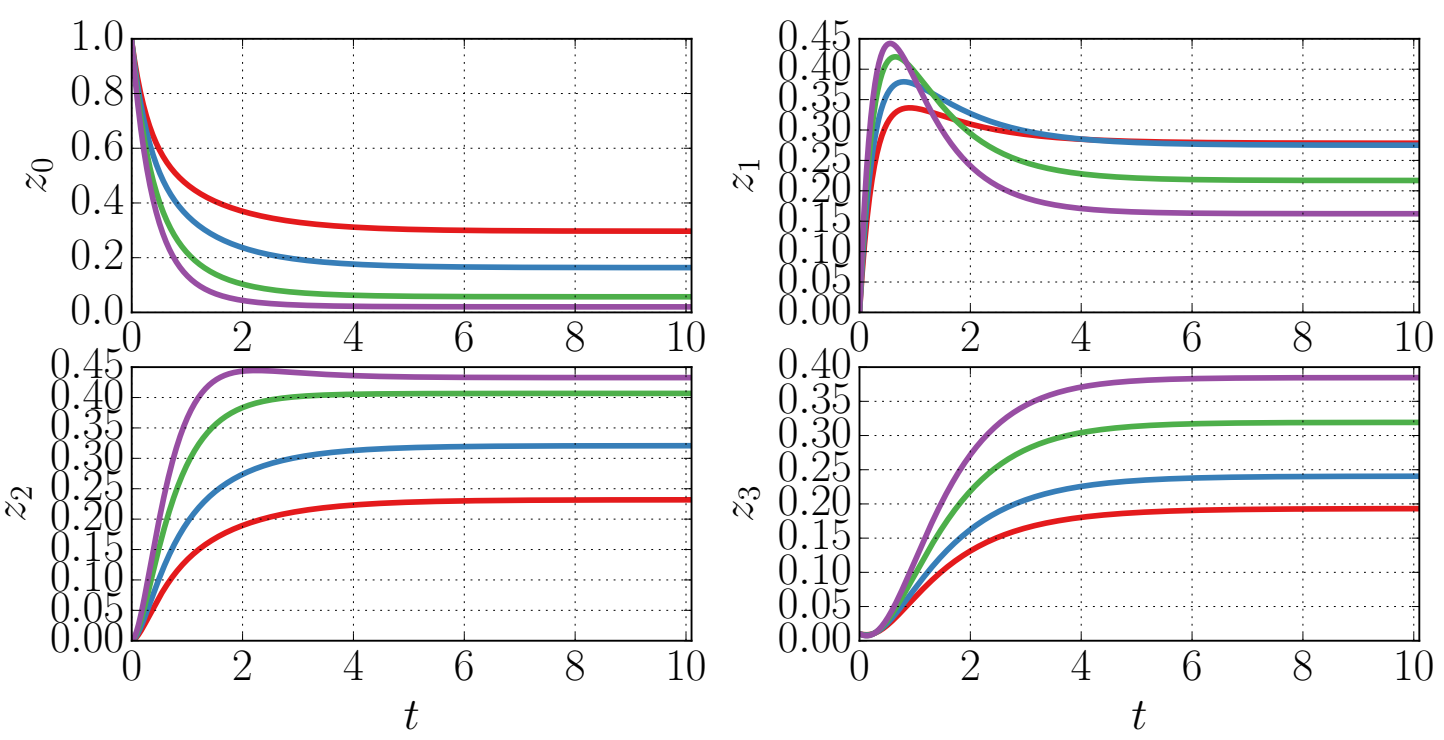

(a) $\xi=1$
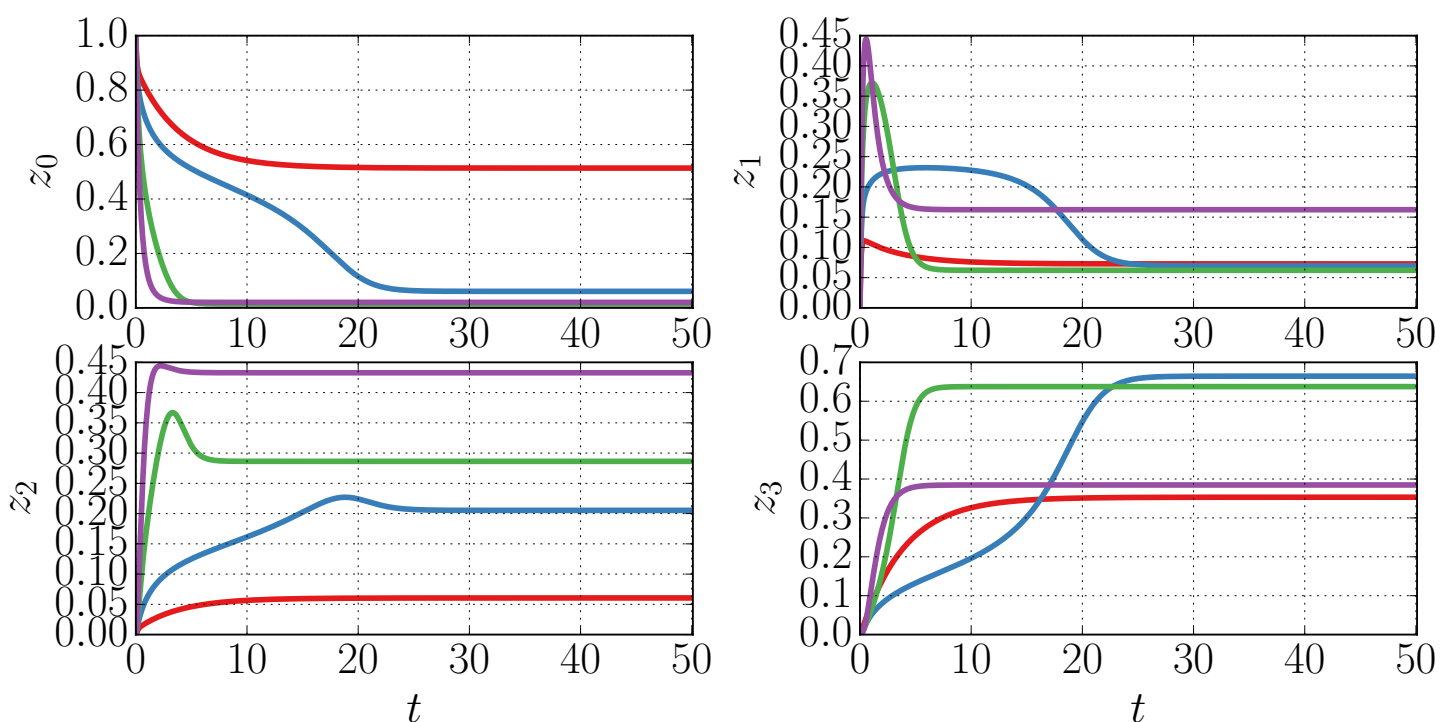

$$
\begin{array}{lll}
\lambda_{p u b}=0 & - & \lambda_{p u b}=0.7 \\
\lambda_{p u b}=0.3 & - & \lambda_{p u b}=1
\end{array}
$$

(b) $\xi=10$

Figure 5.15: Trajectories of $z_{0}, z_{1}, z_{2}$ and $z_{3}$ for different values of $\lambda_{\text {pub }}$ using the unanimity pressure model. Parameters: $\pi_{A}=0.8, \pi_{B}=0.3, \pi_{a}=0.5, \pi_{b}=0.6$

When $\xi$ is high, different combinations of $\lambda_{p u b}$ and attractiveness of $A$ and $B$ in public and private situations sometimes make the dynamics dependent on the initial condition. One such example is shown in fig. 5.16. For $\xi=1$ (solid lines), both initial conditions lead to the same final fraction. However, when $\xi=10$ (dashed lines), we see that low initial $z_{A}$ produces a low final $z_{A}$ level and that a high initial $z_{A}$ produces a high final $z_{A}$ level. 


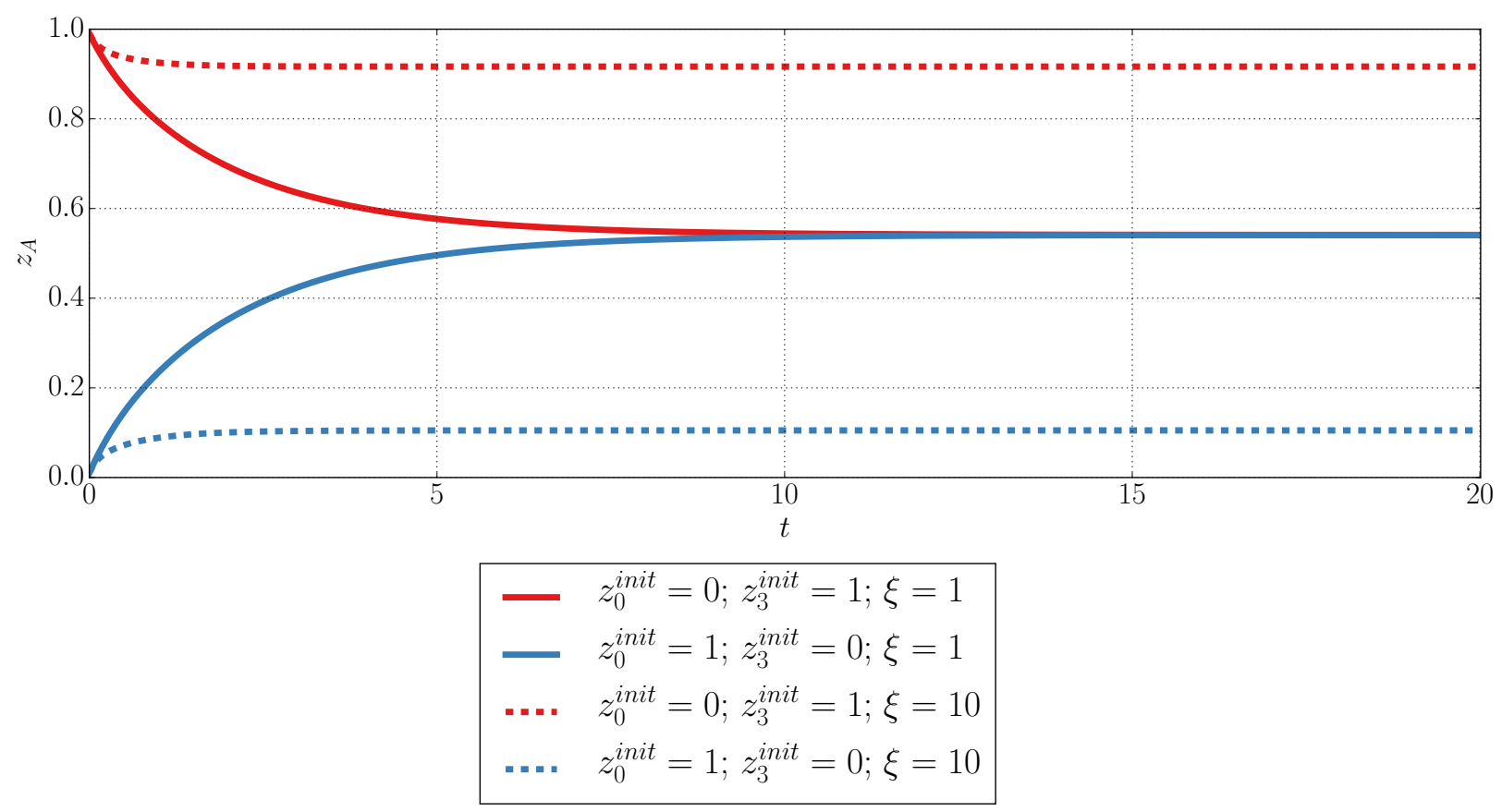

Figure 5.16: Fractions of $A$ speakers over time for different initial conditions and different $\xi$ for the unanimity pressure model when $\lambda_{p u b}=0.4$ and $\lambda_{\text {priv }}=0.6$. The red lines represent the trajectories when the population is initially composed only by group types with only $A$ speakers. The blue lines represent the opposite situation, in which the population is initially composed of group types with only $B$ speakers. Solid lines and dashed lines correspond to the dynamics with $\xi=1$ and $\xi=10$, respectively. Other parameters: $\pi_{A}=0.6, \pi_{B}=0.5, \pi_{a}=0.2, \pi_{b}=0.2$.

We compared how this initial condition dependence affects the equilibrium and the result is shown in fig. 5.17. In these figures, the blue points are stable equilibria and the red crosses are the unstable or approximate saddle points, for six different values of $\lambda_{p u b}$. For intermediate values of $\lambda_{p u b}$, we see that for values of $\pi_{A}$, in a range close to $\pi_{B}$, there are two stable points. As $\lambda_{p u b}$ decreases or increases, this region with two stable points decreases, so that in the extremes there is only a single equilibrium for any $\pi_{A}$ value.

\subsection{Discussion}

In this chapter, we studied the competition dynamics between a local dialect and a standard language. We created a mathematical model to understand how dialect adoption changes when individuals are allowed to choose dialects for two different situations, public and private. Our results showed that for the case in which the two processes (public and private) are completely separated, what determines if a dialect or a standard language will prevail in each situation is how much 


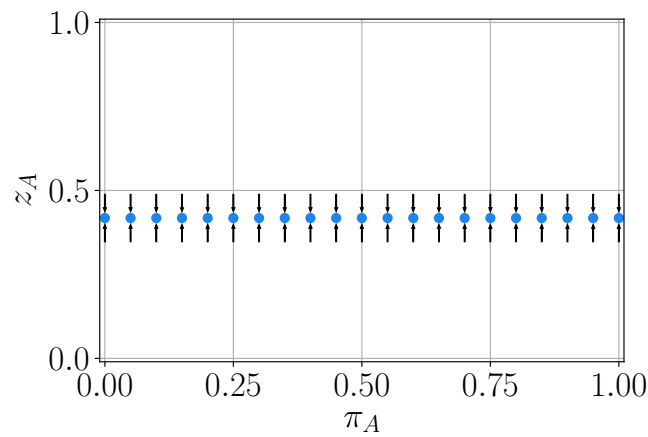

(a) $\lambda_{p u b}=0$ (only private)

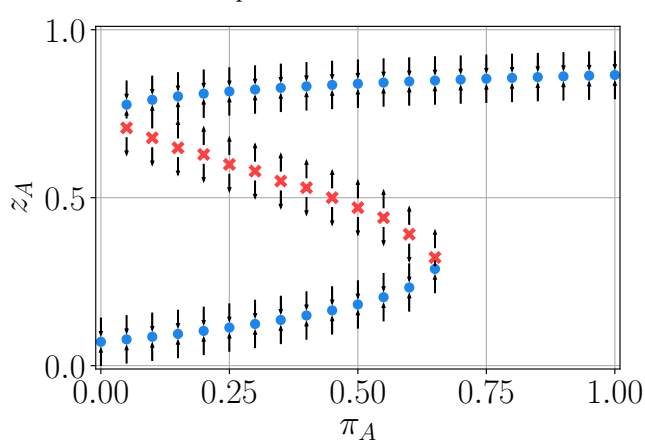

(c) $\lambda_{p u b}=0.4$

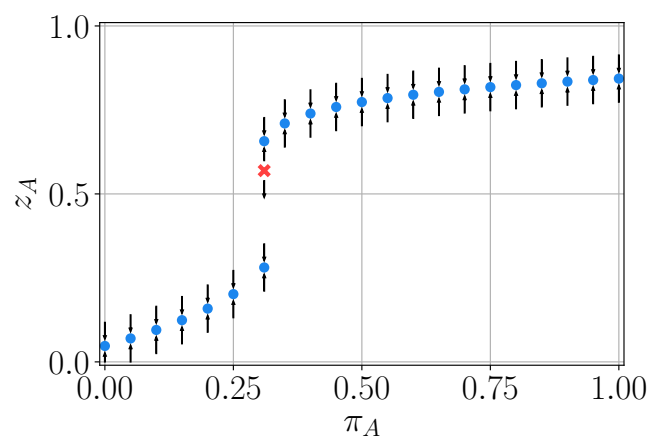

(e) $\lambda_{p u b}=0.8$

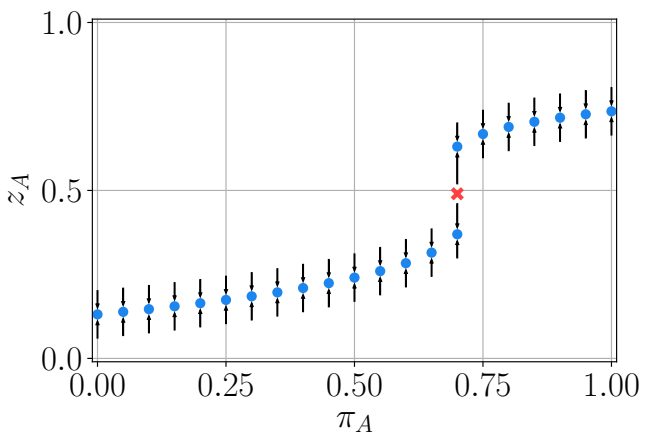

(b) $\lambda_{p u b}=0.2$

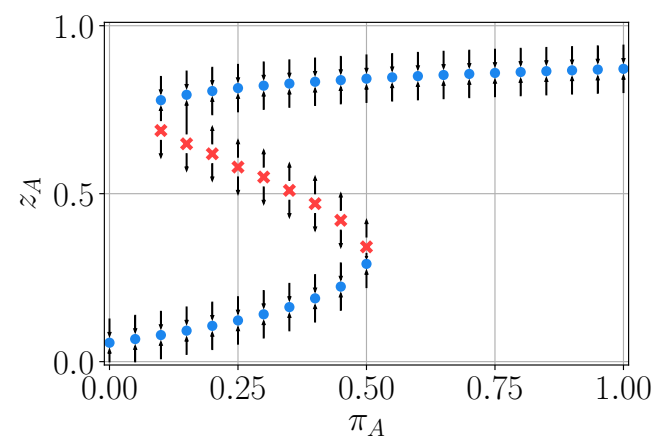

(d) $\lambda_{p u b}=0.6$

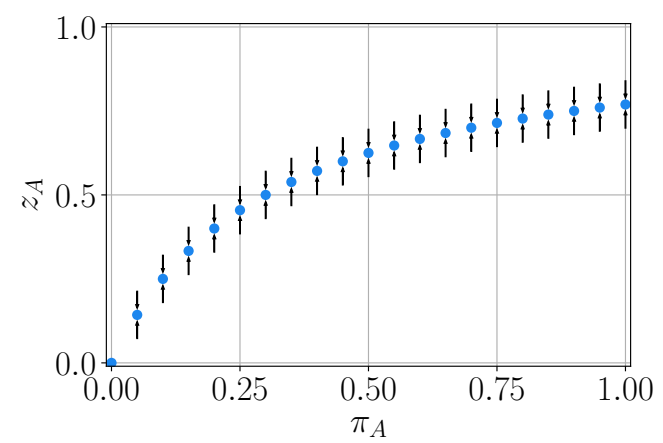

(f) $\lambda_{p u b}=1$ (only public)

Figure 5.17: Equilibrium points $z_{A}$ in function of $\pi_{A}$ for different $\lambda_{\text {pub }}$. Blue points represent the stable equilibrium points, while red crosses represent unstable equilibrium points or approximate saddle points. Parameters: $\pi_{B}=0.3, \pi_{a}=0.5, \pi_{b}=0.6, \xi=10$

individuals are attracted to the dialect or to the standard language. However, conformity may play a role in the private situation, if individuals actions are ruled by the unanimity pressure model, in which individuals are willing to conform only if all the other individuals in the group speak another dialect. In this case, conformity affects both the convergence time and the equilibrium fraction of speakers of each dialect, but only enhances the effect dictated by the attractiveness parameters for the local dialect and the standard language.

We also studied the other extreme case in which the public and private language dynamics are coupled - when no individuals who speak different dialects in public and private are allowed in the population. In this case, for the unanimity pressure model, we found that depending on how much 
influence is placed in public/private situations, when conformity is high, some sets of parameters may lead to dynamics that depend on the initial conditions. In particular, it is possible that a local dialect prevail over the standard language, even if more importance is placed on the public situations and if there is higher attractiveness for the standard language in public $\left(\pi_{A}>\pi_{B}\right)$. This occurs when, initially, the majority of speakers use the local dialect in public and the attractiveness for the local dialect, in private, is not lower than the one for the standard language.

One interesting result from our models was that conformity level only affects the stable fractions of dialect speakers in the unanimity pressure setting. In fact, there has been some studies on conformity that supports the unanimity pressure over our peer pressure setting. Asch $[8,9]$ conducted several experiments to test how individuals conform their opinion to the others' opinions. He designed a simple experiment that consisted of asking individuals to choose among three lines with different sizes which one corresponded to the line shown separately. The three lines were clearly different from one another and it was easy for anyone to spot the correct answer. However, few individuals would always sustain the correct answer when confronted with a majority carrying a different opinion. Testing with the size of majority revealed that: (i) the size of majority only affects the conformity levels until a certain threshold; (ii) having a partner to agree with you (breaking the unanimity) makes individuals more prone to sustain their original opinion. These results could indicate that unanimity pressure is strongly felt by individuals inside a group. It also suggests a more realistic peer pressure setting would be to make individuals more prone to conform only when they are against the majority inside a group.

In China and in Japan, there has been a change in the use of dialects, since the introduction of policies to spread a standard language, around the end of 19th century in Japan [88] and the beginning of 20th century, in China [12]. Although in Japanese language, there exists a subtle difference between what was enforced as the standard language and the common language used nowadays [88], we here consider them to be the same.

Some researchers have focused in tracing the use of different dialects across time. For instance, in the case of Chongqing dialect (China), although its use has declined along the years, it has been kept as the primary language for communication within family and friends [95]. In Japan, one study [47] asked individuals to report their use of the local dialect versus the standard language in different situations, that could be divided into public and private categories. In the results, there 
was a common pattern of using frequently the local dialect in private interactions while rarely using it in public interactions.

However, Kansai dialect (from Osaka region) is a singular case: many individuals stated that they used the local dialect frequently even for some situations considered to be public. One possibility for the reason why Kansai dialect is so different from the other dialects could be related to a higher cost associated with the use of different ways of speaking in public and private. This would make the language dynamics more similar to our coupled dynamics, in which individuals always use only one of the languages to communicate.

Also, another Japanese survey [3] asked individuals to report their preferences regarding their dialect and whether they use the dialect in private but the standard language in public. In general, most individuals reported that they do not use the dialect in public and revealed preference toward the local dialect. These results indicate that the attachment to the local dialect is higher for private interactions than for public interactions, in which the standard language dominates.

In this survey, Okinawan dialect stands out, as more than $90 \%$ of individuals answered that they liked their dialect. It is interesting to observe that these individuals also like the standard language as well and more than $80 \%$ of them said they separate the use of local dialect and standard language or do not use the local dialect at all. One possibility is that this reflect some negative feeling about the use their dialect in public. In fact, the number of Okinawan people who liked their dialect was lower before, but NHK surveys show that since 1978, probably due to campaigns promoting Okinawan dialect, this number has been steadily increasing while the number of people that reported using the local dialect with family and friends has been increasing [3].

This case, in combination with our model results, suggests that if we want one local dialect to persist in the presence of a national standard language, one way to do it is to enforce public policies that promote the local dialect pride, so that we can increase the preference of individuals toward their local dialect. 


\section{Chapter 6}

\section{Conclusions and perspectives}

In this thesis, we studied two social evolution problems: the emergence of altruism when there are intergroup wars, and the persistence of local dialects in the presence of a national standard language. Both problems are inherent of human nature and can be seen as part of the areas of evolution of cooperation and cultural evolution.

In the first problem we tackled the question of emergence. We were concerned with the question of how could altruism emerge in a hunter-gatherers society when there were likely frequent intergroup wars. To investigate that question, we created a mathematical model based on the framework TwoLevel Fisher Wright under the assumption of weak selection. We created a mathematical model and performed numerical simulations along with an agent-based simulation that confirmed that intergroup wars have possibly played a role in the emergence of human altruism.

While the proposed model is general in its structure, some assumptions could be changed in order to analyze other aspects of the problem. One example is the assumption that $\beta$ is constant. It would be interesting to analyze what happens when $\beta$ changes as a function of time, since groups may not always be at war with each other. Another direction could be to incorporate a selection strength parameter explicitly into the model. This could be done by redefining the fitness functions similarly to what was done in chapter 3 .

In the second problem, we modeled a different part of evolution, and focused on the persistence of a language. In this case, we were interested not only in finding an explanation to what happens in some local communities which preserve their local dialects, despite having a national language reinforced by a central authority, but also we expected our model could indicate ways to increase 
the chances of survival of that local dialects. Our results suggest that if we want one local dialect to persist in the presence of a national standard language, one way to do it is to enforce public policies that promote the local dialect pride, so that we can increase the preference of individuals toward their local dialect.

We can see two interesting further directions for this model. One of them concerns relaxing the assumption of extremely high or low cost of using two languages, so that we can calculate how the dynamics changes when the cost is at an intermediate value and allows individuals to use separate languages at different types of interaction. Another direction would be to think of how to accommodate variations in dialect preference levels along time, since environmental and cultural changes, that could arise either naturally or motivated by public policies, might have some impact in the way dialects are perceived by individuals. 
Appendices 


\section{Appendix A}

\section{Short introduction to evolutionary game}

\section{theory}

The development of the field of Game Theory is usually attributed to von Neumann and Morgerstern, although Borel had also delineated some of the ideas of this theory in early 1920s [104]. Game theory has been used primarily in Economics, and is divided into two subdisciplines: cooperative game theory and non-cooperative game theory. Evolutionary game theory has been developed from the non-cooperative game theory, thus, we will focus on explaining the basic notions of noncooperative games before introducing the concepts and equations behind evolutionary game theory. The following paragraphs are based on basic concepts of game theory and evolutionary game theory found in $[40,90,103,70]$.

\section{A.1 Basic notions of game theory}

In this section, we provide basic notions of game theory, both formally and informally. First, we start from the definition of a game and the different types of strategies a player may adopt. Then, we explain how to analyze a game using the concept of Nash Equilibrium.

In non-cooperative game theory, in order to describe a game, we need three elements: the number of players, possible actions (strategies) for each player and payoffs, which can vary according to what actions were played and who played them. Many daily situations can be modeled as games; common examples include: matching pennies, rock-paper-scissors etc. The key part of a game is 
the interaction among players. Thus, not every situation can be modeled as a game. For instance, peg solitaire is not a game in the sense of game theory.

More formally, we define a game as the set $(N, S, V)$, in which, $N$ is the number of players; $S$ is the space of strategies in the game, $S=\times_{i \in I} S_{i}$, where $I=\{1,2, \ldots, N\}$ and $S_{i}$ is the set of $k_{i}$ strategies of player $i$; and $V$ is the matrix containing the payoff values for each interaction. The vector $\left(s_{1}, s_{2}, \ldots, s_{N}\right)$, which consists of all strategies $s_{i} \in S_{i}$ played, for each player $i=$ $1,2, \ldots, N$, is called a strategy profile. Payoffs matrix $V$ is built by applying a payoffs function $f:\left(s_{1}, s_{2}, \ldots, s_{N}\right) \rightarrow \mathbb{R}^{N}$. A simplified template of a game is shown in fig. A.1.

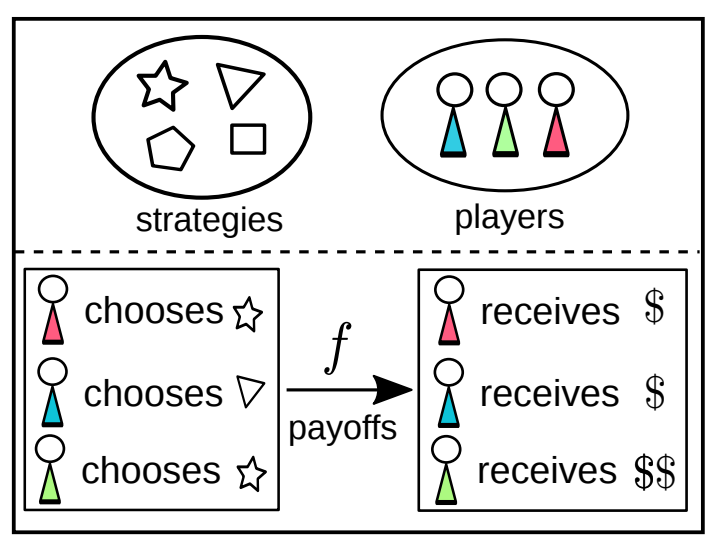

Figure A.1: Necessary elements of a game: a set of strategies, number of players and a payoff function $f$. For illustrative purposes, here we limit $N=3$ players, we consider all the players have the same set of strategies and we show a map for only one strategy profile.

Note that when players deterministically choose which strategy to use, we say they are using pure strategies. It also would be possible for a player to randomize their strategies. In this case, players choose their strategies according to a probability distribution, leading to an infinite number of combinations. This probability distribution is what is commonly referred as a mixed strategy.

Definition A.1. Mixed strategy. Suppose a player $i$ has $k_{i}$ strategies: $s_{1}, s_{2}, \ldots, s_{k_{i}}$., for $s_{i} \in S$ A mixed strategy is a strategy in which the player $i$ chooses the pure strategy $s_{i j}$ with probability $p_{i j}$, for $j=1,2, \ldots, k_{i}$. Hence, it is a probability distribution over the set of pure strategies, with the following form:

$$
\sigma_{i}=\left(p_{i 1}, p_{i 2}, \ldots, p_{i k_{i}}\right)
$$

in which $\sum_{j=1}^{k_{i}} p_{i j}=1$.

If $p_{i j}=0$ for a particular $j$, and $p_{i m}=0$ for every $m \neq j$, then $\sigma_{i}$ is a pure strategy. If $p_{i j}>0, \forall j$, then all strategies are used and $\sigma_{i}$ is said to be a completely mixed strategy. 
A core concept is game theory is the Nash equibrium. Informally, a Nash equilibrium is defined as the action from which the players do not have incentive to deviate. This means that if no other information is given, they will stick to the chosen strategy, because individually changing the strategy is not advantageous.

More formally, to define Nash equilibrium, we shall use the concept of best response. A best response is defined in relation to a strategy profile $y \in \Theta$. It is the strategy such that no other strategy can give a strictly higher payoff.

Definition A.2. Best response. Let $\pi_{i}: S \rightarrow \mathbb{R}$ be the payoff function for player $i$, and let $y_{-i}$ represents the strategy profile of the players other than $i$. A best response correspondence is given by:

$$
\tilde{\beta}_{i}(y)=\left\{x_{i} \in \Delta_{i}: \pi\left(x_{i}, y_{-i}\right) \geq \pi\left(z_{i}, y_{-i}\right) \quad \forall z_{i} \in \Delta_{i}\right\} .
$$

The combined best response correspondence is just the cartesian product of all players' correspondences: $\tilde{\beta}(y)=\times_{i \in I} \tilde{\beta}_{i}(y) \subset \Theta$. From this, the definition of Nash equilibrium follows:

Definition A.3. Nash equilibrium. A strategy profile $\sigma \in \Theta$ is a Nash equilibrium if $\sigma \in \tilde{\beta}(\sigma)$, i.e., $\sigma$ is a Nash equilibrium if it is a best response to itself.

\section{A.1.1 Public good games}

Public goods are ubiquitous in human societies. However, their existence is subject to a problem called the Tragedy of the Commons [44]: it is always advantageous to overuse a public resource, hence the public resource may be depleted because of the continuous overuse.

The games classified as public goods games model the interaction of the individuals that share a common resource. In the classical public goods game, $n$ individuals contribute to a common resources pool. They can either cooperate (C), by contributing to the pool a fixed value $c$, or defect (D), by not contributing. The sum of the individual contributions is then multiplied by a factor $\alpha>0$ and divided among all individuals in the population. Hence, defectors are free-riders: they get the benefit without cost.

This basic public goods game can be divided into two types, according to how the contribution share is calculated: self-return public goods game (SRPG) or others-only public goods game (OOPG) [90]. 
Let $n$ be the number of individuals in the population and $k$ be the number of cooperators. In SRPG, only cooperators pay a cost $c$, but all individuals $(C$ or $D)$ receive an equal share of the total amount $(b k / n)$. In OOPG, the cooperator does not receive her own share, receiving $b(k-1) /(n-1)$, and pays the same cost $c$. On the other hand, a defector receives payoff $b k /(n-1)$. In both cases, $b$ is the marginal benefit of the game, which is the product of the multiplier $\alpha$ with the cost $c$, i.e., $b=\alpha c>0$. These payoffs are represented in table A.1.

\begin{tabular}{|c|c|c|c|}
\hline Game & $v_{k}^{C}$ & $v_{k}^{D}$ & Parameters \\
\hline SRPG & $b k / n-c$ & $b k / n$ & $b>0, c>0$ \\
OOPG & $b(k-1) /(n-1)-c$ & $b k /(n-1)$ & $b>0, c>0$ \\
\hline
\end{tabular}

Table A.1: Summary table with the payoffs for SRPG and OOPG.

Note that if we denote the benefit in OOPG as $B$ and the benefit in SRPG as $b$, we can find a relation between them. Given that the cost $c$ is the same for both games, we can see that $B=\frac{(n-1) b}{n}$. Thus, results obtained for OOPG (or conversely, SRPG) can be easily extended to the other public goods game.

In both games, if the population is very large, since the payoff of a defector is always greater than the one of a cooperator, cooperation never wins. One way to make cooperation feasible is to incorporate repeated interactions between the players into the game. There are many ways to do that, including the addition of a punisher type of player who can punish those who free ride, for example. Another simple model has become known as Iterated Public Goods Game (IPG) and is based on the mechanism of reciprocity [49, 22].

IPG can be considered one possible generalization (in the number of players) of TFT (tit-for-tat game), presented in section 2.3.2 of the main text. In the two-players version of TFT, individuals play cooperate in the current round if the other player cooperated in the previous round of the game. In the first round, it is common to assume individuals always cooperate. For the $n$-players version, we keep the same assumption of cooperativeness in the first round. Then, individuals can be conditional cooperators $(C)$ or defectors $(D)$. Conditional cooperators will cooperate in other rounds by looking at the immediate previous round cooperation level: if the number of cooperators $k$ was larger than a threshold $\theta \geq 0$, then individuals cooperate by paying a cost $c$ and receive a benefit $b(k-1) /(n-1)$. Defectors, on the other hand, always receive a benefit $b k /(n-1)$, without paying any cost. 
Note that, as in the two-players version of TFT, the number of rounds is not fixed: at any period, the probability of having a further round is given by $0<\omega<1$. This means, on average, any game will have $T$ rounds, with $T=1 /(1-\omega)$. Also, note that future cooperation depends on the initial number of cooperators in the first round. If, in the first round, $k \leq \theta$, benefit is only produced in that first round. On the other hand, if $k>\theta$ in the first round, then, the expected payoff of a cooperator will be the average number of rounds $T$ multiplied to the net benefit $(-c+b(k-1) /(n-1))$ at a single round. Analogously, for a defector, the expected payoff would be $T b k /(n-1)$.

Hence, the payoffs for $C$ and $D$ in IPG are given by:

$$
\begin{aligned}
& v_{k}^{C}=\left\{\begin{array}{cc}
-c+\frac{b(k-1)}{n-1}, & \text { if } k \leq \theta, \\
T\left(-c+\frac{b(k-1)}{n-1}\right), & \text { if } k>\theta,
\end{array}\right. \\
& v_{k}^{D}= \begin{cases}\frac{b k}{n-1}, & \text { if } k \leq \theta, \\
T \frac{b k}{n-1}, & \text { if } k>\theta .\end{cases}
\end{aligned}
$$

In $[49,22]$, it was found that cooperators could coexist with defectors if certain conditions are met, for instance, a sufficient large number of cooperators. In particular, [22] investigated the relation between group size and reciprocal cooperation and found that under random formation of groups, the set of conditions which make cooperation attainable in sizable groups is very restricted, which implied further explanations would be necessary to explain cooperation as seen among humans.

Another common public goods game is called Volunteer's Dilemma (VD)[32]. This is a modified n-person Prisoner's Dilemma (NPD) in which individuals who are suspects of committing a crime are offered two options: cooperate by volunteering to confess or defect by remaining silent. If none of them volunteers, all of them goes to jail for ten years; if at least one of them volunteers and confess the crime, those who volunteered go to jail for one year, while the others who defected are released with no jail time.

This game has also been called Threshold Public Goods Game (TPG or THR) (e.g. in [27]) or Step-Level Public Goods Game (e.g. in [80]) because of the nature of the payoff functions. In the main text, we adopted the name Threshold Public Goods Game (THR) to refer to this game.

In the general case of $\mathrm{VD}$, we consider benefit is produced whenever the number $k$ of volunteers is at least $\theta$; for instance, in the above example of the prisoners, $\theta=1$. Cooperators receive benefit 
$b>0$ (if $k \geq \theta$ ) and are inflicted a cost $c>0$ while defectors receive a benefit $b^{\prime}>0$ (if $k \geq \theta$, which may be different from the benefit received by cooperators (as, for instance, in the case of VD as described by [32]). The payoffs for $C$ and $D, v_{k}^{C}$ and $v_{k}^{D}$, depend on the number $k$ of volunteering individuals and are given by:

$$
\begin{gathered}
v_{k}^{C}= \begin{cases}-c, & \text { if } k<\theta, \\
-c+b, & \text { if } k \geq \theta,\end{cases} \\
v_{k}^{D}= \begin{cases}0, & \text { if } k<\theta, \\
b^{\prime}, & \text { if } k \geq \theta .\end{cases}
\end{gathered}
$$

An interesting result about VD and public goods game, in general, can be found in [7], which showed VDs, in contrast to the original version of NPD, can lead to equilibrium frequencies in which both $C$ and $D$ coexist. This result is particularly important, since it proved that it is possible to achieve cooperation even in the absence of repeated interactions and regardless of the relatedness level among individuals.

\section{A.2 Basic notions of evolutionary game theory}

Evolutionary game theory started from the work of Maynard Smith and George R. Price [92], who published, in 1973, a paper about animal conflict strategies. Inspired by the (unpublished) paper of Price on the advantage of the retaliation strategy in intraspecific conflicts [89], Smith was responsible for the development of the concept of Evolutionary Stable Strategies. According to them, an evolutionary stable strategy (ESS) "is a strategy such that, if most of the members of a population adopt it, there is no 'mutant' strategy that would give higher reproductive fitness" [92].

While non-cooperative game theory is focused on games at individuals level, evolutionary game theory is concerned with games at population level, so that now strategy profiles $x=\left(x_{1}, x_{2}, \ldots, x_{n}\right)$ identify the fraction $x_{i}$ of the population which play each pure-strategy. Furthermore, calculating the payoffs for evolutionary games means averaging the payoffs over all individuals inside the population, i.e., calculating expected payoffs.

Definition A.4. Let $\mathrm{E}_{x}[y]$ be the expected payoff from playing $y$ against $x$. A strategy $a$ is an ESS if:

$$
\mathrm{E}_{a}[a]>\mathrm{E}_{a}[b], \quad \forall b \neq a
$$


If, however, $\mathrm{E}_{a}[a]=\mathrm{E}_{a}[c]$, for some $c$, then, $a$ is an $\operatorname{ESS}$ if:

$$
\mathrm{E}_{c}[a]>\mathrm{E}_{c}[c]
$$

The ESS is a refinement of a Nash equilibrium, in the sense that it is a strict Nash equilibrium. While the payoff obtained by a Nash equilibrium strategy profile should only be higher or equal to the payoff calculated for other strategy profiles, in the case of an ESS, the relation is strict: the payoff should be higher than all other payoffs.

Peter Taylor and Leo Jonker [97] were the ones responsible for linking a dynamics to the concept of ESS. They proposed a differential equation that allows us to follow the evolution of strategies frequencies in a population. This is equation is called replicator equation.

Taylor and Jonker assumed a game in which $n$ pure strategies, $s_{1}, s_{2}, \ldots, s_{n}$, are available for the individuals of a population. At each period $t$, the state of the population is given by a vector $\left(x_{1}, x_{2}, \ldots, x_{n}\right)$, in which each coordinate $x_{i}$ is the frequency of individuals playing the pure strategy $s_{i}$. Also, let $f_{i}$ be the expected payoff from playing $s_{i}$ and suppose it represents the incremental effect on an individual's fitness. Then, the replicator equation is given by Eq. A.8.

$$
\frac{d x_{i}}{d t}=x_{i}\left(f_{i}-\phi\right)
$$

in which $\phi=\sum_{i=1}^{n} x_{i} f_{i}$ is the average expected payoff. 


\section{Bibliography}

[1] More than 19,000 customers 'pay it forward' in Starbucks South China stores. https: //web.archive.org/web/20150910083059/https://news. starbucks. com/news/morethan-19000-customers-pay-it-forward-in-starbucks-south-china-stores.

[2] D. M. Abrams and S. H. Strogatz. Linguistics: Modelling the dynamics of language death. Nature, 424(6951):900-900, 2003.

[3] M. Aizawa. Research on present-day dialect consciousness: nationalwide survery in 2010 and its statistical analysis (in Japanese). NINJAL Project Review, 3(1):26-37, 2012.

[4] M. W. Allen and T. L. Jones. Violence and warfare among hunter-gatherers. Left Coast Press, 2014.

[5] T. Amano, B. Sandel, H. Eager, E. Bulteau, J.-C. Svenning, B. Dalsgaard, C. Rahbek, R. G. Davies, and W. J. Sutherland. Global distribution and drivers of language extinction risk. Proceedings of the Royal Society of London B: Biological Sciences, 281(1793):20141574, 2014.

[6] A. M. Andres, M. Soldevila, A. Navarro, K. K. Kidd, B. Oliva, and J. Bertranpetit. Positive selection in MAOA gene is human exclusive: determination of the putative amino acid change selected in the human lineage. Human genetics, 115(5):377-386, 2004.

[7] M. Archetti and I. Scheuring. Coexistence of cooperation and defection in public goods games. Evolution, 65(4):1140-1148, 2011.

[8] S. E. Asch. Opinions and social pressure. Scientific American, 193(5):31-35, 1955.

[9] S. E. Asch. Studies of independence and conformity: I. a minority of one against a unanimous majority. Psychological monographs: General and applied, 70(9):1, 1956.

[10] R. Axelrod. The dissemination of culture a model with local convergence and global polarization. Journal of conflict resolution, 41(2):203-226, 1997. 
[11] R. Axelrod and W. D. Hamilton. The evolution of cooperation. Science, 211(4489):1390-1396, 1981.

[12] J. Bai. Language attitude and the spread of standard chinese in china. Language Problems and Language Planning, 18(2):128-138, 1994.

[13] A. Baronchelli. The emergence of consensus: a primer. Royal Society Open Science, 5(2): $172189,2018$.

[14] M. E. Borrello. The rise, fall and resurrection of group selection. Endeavour, 29(1):43-47, 2005.

[15] A. F. Bourke. Principles of social evolution. Oxford University Press Oxford, 2011.

[16] S. Bowles. Group competition, reproductive leveling, and the evolution of human altruism. science, 314(5805):1569-1572, 2006.

[17] S. Bowles. Did warfare among ancestral hunter-gatherers affect the evolution of human social behaviors? Science, 324(5932):1293-1298, 2009.

[18] S. Bowles. Microeconomics: behavior, institutions, and evolution. Princeton University Press, 2009.

[19] S. Bowles and H. Gintis. A cooperative species: Human reciprocity and its evolution. Princeton University Press, 2011.

[20] R. Boyd and J. P. Lorberbaum. No pure strategy is evolutionarily stable in the repeated prisoner's dilemma game. Nature, 327(6117):58, 1987.

[21] R. Boyd and P. J. Richerson. Culture and the evolutionary process. University of Chicago Press, 1988.

[22] R. Boyd and P. J. Richerson. The evolution of reciprocity in sizable groups. Journal of theoretical Biology, 132(3):337-356, 1988.

[23] R. Boyd and P. J. Richerson. The evolution of indirect reciprocity. Social Networks, 11(3): 213-236, 1989

[24] R. Boyd, R. H. Schonmann, and R. Vicente. Hunter-gatherer population structure and the evolution of contingent cooperation. Evolution and Human Behavior, 35(3):219-227, 2014. 
[25] H. Brandt, H. Ohtsuki, Y. Iwasa, and K. Sigmund. A survey of indirect reciprocity. In Mathematics for ecology and environmental sciences, pages 21-49. Springer, 2007.

[26] H. G. Brunner, M. Nelen, X. Breakefield, H. Ropers, and B. Van Oost. Abnormal behavior associated with a point mutation in the structural gene for monoamine oxidase A. Science, 262(5133):578-580, 1993.

[27] C. B. Cadsby and E. Maynes. Voluntary provision of threshold public goods with continuous contributions: experimental evidence. Journal of Public Economics, 71(1):53-73, 1999.

[28] L. L. Cavalli-Sforza and M. W. Feldman. Cultural transmission and evolution: a quantitative approach. Number 16. Princeton University Press, 1981.

[29] D. Crystal. How language works. Penguin UK, 2007.

[30] J. Damuth and I. L. Heisler. Alternative formulations of multilevel selection. Biology and Philosophy, 3(4):407-430, 1988.

[31] R. Dawkins. The Selfish Gene - with a new Introduction by the Author. Oxford University Press, USA, 2006.

[32] A. Diekmann. Volunteer's dilemma. Journal of conflict resolution, 29(4):605-610, 1985.

[33] L. A. Dugatkin. The altruism equation: Seven scientists search for the origins of goodness. Princeton University Press, 2011.

[34] J. Farrell and R. Ware. Evolutionary stability in the repeated prisoner's dilemma. Theoretical population biology, 36(2):161-166, 1989.

[35] S. Freeman and J. C. Herron. Evolutionary analysis. Pearson Prentice Hall, 4th edition edition, 2007.

[36] A. Gardner. The price equation. Current Biology, 18(5):R198-R202, 2008.

[37] A. Gardner. The genetical theory of multilevel selection. Journal of evolutionary biology, 28 (2):305-319, 2015.

[38] A. Gat. War in human civilization. Oxford University Press, 2008.

[39] Y. Gilad, S. Rosenberg, M. Przeworski, D. Lancet, and K. Skorecki. Evidence for positive selection and population structure at the human MAO-A gene. Proceedings of the National Academy of Sciences, 99(2):862-867, 2002. 
[40] H. Gintis. Game theory evolving: A problem-centered introduction to modeling strategic behavior. Princeton university press, 2000.

[41] C. Goodnight. Multilevel selection theory and evidence: a critique of gardner, 2015. Journal of evolutionary biology, 28(9):1734-1746, 2015.

[42] W. D. Hamilton. Narrow Roads of Gene Land: Volume 2: Evolution of Sex, volume 2. Oxford University Press, 2001.

[43] D. Hamilton William. Genetical evolution of social behavior i and ii. Journal of Theoretical Biology, (7):1-52, 1964.

[44] G. Hardin. The tragedy of the commons. Science, 162(3859):1243-1248, 1968.

[45] T. Harris. The theory of branching processes. 1963.

[46] E. Haugen. Dialect, language, nation. American Anthropologist, 68(4):922-935, 1966.

[47] F. Inoue. New dialect and standard language. style shift in tokyo. Area and Cultural Studies, 42:49-68, 1991.

[48] F. Inoue. The significance of new dialects. Dialectologia et Geolinguistica, 1:3-27, 1993.

[49] N. Joshi. Evolution of cooperation by reciprocation within structured demes. Journal of Genetics, 66(1):69-84, 1987.

[50] L. H. Keeley. War before civilization. OUP USA, 1997.

[51] R. C. Kelly. Warless societies and the origin of war. University of Michigan Press, 2000.

[52] B. Kerr, P. Godfrey-Smith, and M. W. Feldman. What is altruism? Trends in ecology $\mathcal{E}$ evolution, 19(3):135-140, 2004.

[53] D. Krupp. How to distinguish altruism from spite (and why we should bother). Journal of evolutionary biology, 26(12):2746-2749, 2013.

[54] M. M. Lahr, F. Rivera, R. Power, A. Mounier, B. Copsey, F. Crivellaro, J. Edung, J. M. Fernandez, C. Kiarie, J. Lawrence, et al. Inter-group violence among early holocene huntergatherers of west turkana, kenya. Nature, 529(7586):394-398, 2016.

[55] P. M. Lambert. Patterns of violence in prehistoric hunter-gatherer societies of coastal southern california. Troubled times: Violence and warfare in the past, pages 77-110, 1997. 
[56] S. A. LeBlanc and K. E. Register. Constant battles: Why we fight. Macmillan, 2004.

[57] L. Lehmann and L. Keller. The evolution of cooperation and altruism-a general framework and a classification of models. Journal of evolutionary biology, 19(5):1365-1376, 2006.

[58] L. Lehmann, K. Bargum, and M. Reuter. An evolutionary analysis of the relationship between spite and altruism. Journal of evolutionary biology, 19(5):1507-1516, 2006.

[59] L. Lehmann, L. Keller, S. West, and D. Roze. Group selection and kin selection: two concepts but one process. Proceedings of the National Academy of Sciences, 104(16):6736-6739, 2007.

[60] S. B. Manuck, J. D. Flory, R. E. Ferrell, J. J. Mann, and M. F. Muldoon. A regulatory polymorphism of the monoamine oxidase-A gene may be associated with variability in aggression, impulsivity, and central nervous system serotonergic responsivity. Psychiatry research, 95(1): 9-23, 2000 .

[61] D. G. Mayo and N. L. Gilinsky. Models of group selection. Philosophy of Science, pages $515-538,1987$.

[62] R. McDermott, D. Tingley, J. Cowden, G. Frazzetto, and D. D. Johnson. Monoamine oxidase a gene (MAOA) predicts behavioral aggression following provocation. Proceedings of the National Academy of Sciences, 106(7):2118-2123, 2009.

[63] R. McElreath and R. Boyd. Mathematical models of social evolution: A guide for the perplexed. University of Chicago Press, 2008.

[64] C. D. Meyer. Matrix analysis and applied linear algebra. Siam, 2000.

[65] R. E. Michod. Darwinian dynamics: evolutionary transitions in fitness and individuality. Princeton University Press, 2000.

[66] J. W. Minett and W. S. Wang. Modelling endangered languages: The effects of bilingualism and social structure. Lingua, 118(1):19-45, 2008.

[67] J. Mira, L. F. Seoane, and J. J. Nieto. The importance of interlinguistic similarity and stable bilingualism when two languages compete. New Journal of Physics, 13(3):033007, 2011.

[68] C. Moseley. Atlas of the world's languages in danger, 2010. URL http://www.unesco.org/ culture/en/endangeredlanguages/atlas. 
[69] M. Nowak, K. Sigmund, et al. A strategy of win-stay, lose-shift that outperforms tit-for-tat in the prisoner's dilemma game. Nature, 364(6432):56-58, 1993.

[70] M. A. Nowak. Evolutionary dynamics. Harvard University Press, 2006.

[71] M. A. Nowak and D. C. Krakauer. The evolution of language. Proceedings of the National Academy of Sciences, 96(14):8028-8033, 1999.

[72] M. A. Nowak and S. Roch. Upstream reciprocity and the evolution of gratitude. Proceedings of the Royal Society of London B: Biological Sciences, 274(1610):605-610, 2007.

[73] M. A. Nowak and K. Sigmund. Evolution of indirect reciprocity by image scoring. Nature, 393(6685):573, 1998.

[74] H. Ohtsuki and Y. Iwasa. The leading eight: social norms that can maintain cooperation by indirect reciprocity. Journal of Theoretical Biology, 239(4):435-444, 2006.

[75] S. Okasha. Evolution and the levels of selection, volume 16. Clarendon Press Oxford, 2006.

[76] E. Ostrom. Governing the commons. Cambridge university press, 2015.

[77] W. Poundstone. Prisoner's Dilemma/John von Neumann, Game Theory and the Puzzle of the Bomb. Anchor, 1993.

[78] G. R. Price. Selection and Covariance. Nature, 227(5257):520-521, Aug. 1970. ISSN 00280836. doi: 10.1038/227520a0. URL http://dx.doi.org/10.1038/227520a0.

[79] A. Rapoport and A. M. Chammah. Prisoner's dilemma: A study in conflict and cooperation, volume 165. University of Michigan press, 1965.

[80] A. Rapoport and D. Eshed-Levy. Provision of step-level public goods: Effects of greed and fear of being gypped. Organizational Behavior and Human Decision Processes, 44(3):325-344, 1989.

[81] S. Rice. Evolutionary Theory: Mathematical and Conceptual Foundations. Sinauer Associates, 2004. ISBN 9780878937028. URL https://books.google.co.uk/books?id=nGpoQgAACAAJ.

[82] R. H. Schonmann and R. Boyd. A simple rule for the evolution of contingent cooperation in large groups. Phil. Trans. R. Soc. B, 371(1687):20150099, 2016. 
[83] R. H. Schonmann, R. Vicente, and N. Caticha. Two-level fisher-wright framework with selection and migration: An approach to studying evolution in group structured populations. arXiv preprint arXiv:1106.4783, 2011.

[84] R. H. Schonmann, R. Vicente, and R. Boyd. Invasion, polymorphic equilibria and fixation of a mutant social allele in group structured populations. arXiv preprint arXiv:1211.3377, 2012.

[85] R. H. Schonmann, R. Vicente, and N. Caticha. Altruism can proliferate through population viscosity despite high random gene flow. PloS one, 8(8):e72043, 2013.

[86] T. Sekiguchi and M. Nakamaru. How inconsistency between attitude and behavior persists through cultural transmission. Journal of theoretical biology, 271(1):124-135, 2011.

[87] T. Sekiguchi and M. Nakamaru. How intergenerational interaction affects attitude-behavior inconsistency. Journal of theoretical biology, 346:54-66, 2014.

[88] M. Shibatani. The languages of Japan. Cambridge University Press, 1990.

[89] K. Sigmund. John Maynard Smith and evolutionary game theory. Theoretical Population Biology, 68(1):7-10, 2005.

[90] K. Sigmund. The calculus of selfishness. Princeton University Press, 2010.

[91] B. Simon, J. A. Fletcher, and M. Doebeli. Towards a general theory of group selection. Evolution, 67(6):1561-1572, 2013.

[92] J. M. Smith and G. Price. The logic of animal conflict. Nature, 246:15, 1973.

[93] E. Szathmáry and J. M. Smith. The major transitions in evolution. WH Freeman Spektrum, 1995.

[94] C. M. Tanaka, J. H. Lee, and Y. Iwasa. The persistence of a local dialect when a national standard language is present: An evolutionary dynamics model of cultural diversity. Bulletin of Mathematical Biology, 2018. doi: 10.1007/s1153.

[95] C. Tang. The use of chinese dialects: Increasing or decreasing? Above and Beyond the Segments: Experimental linguistics and phonetics, pages 302-310, 2014.

[96] P. D. Taylor and S. A. Frank. How to make a kin selection model. Journal of Theoretical Biology, 180(1):27-37, 1996. 
[97] P. D. Taylor and L. B. Jonker. Evolutionary stable strategies and game dynamics. Mathematical biosciences, 40(1):145-156, 1978.

[98] R. L. Trivers. The evolution of reciprocal altruism. The Quarterly review of biology, 46(1): 35-57, 1971.

[99] M. Van Veelen. Group selection, kin selection, altruism and cooperation: when inclusive fitness is right and when it can be wrong. Journal of Theoretical Biology, 259(3):589-600, 2009.

[100] M. Van Veelen, J. García, M. W. Sabelis, and M. Egas. Group selection and inclusive fitness are not equivalent; the price equation vs. models and statistics. Journal of theoretical biology, 299:64-80, 2012.

[101] R. Vicente. Altruísmo, Moralidade e Hierarquia: Modelos Quantitativos. Tese de Livre Docência. Instituto de Matemática e Estatística da Universidade de São Paulo, 2015.

[102] M. J. Wade. A critical review of the models of group selection. The Quarterly Review of Biology, 53(2):101-114, 1978.

[103] J. W. Weibull. Evolutionary game theory. MIT press, 1997.

[104] E. R. Weintraub. Toward a history of game theory, volume 24. Duke University Press, 1992.

[105] S. A. West, A. S. Griffin, and A. Gardner. Social semantics: altruism, cooperation, mutualism, strong reciprocity and group selection. Journal of evolutionary biology, 20(2):415-432, 2007.

[106] D. S. Wilson and E. O. Wilson. Rethinking the theoretical foundation of sociobiology. The Quarterly review of biology, 82(4):327-348, 2007. 